Supporting Information

for

\title{
Lateral Distribution of Charged Species along a Polyelectrolyte Probed with a Fluorescence Blob Model
}

\author{
Christine Keyes, Manoj Mathew, Jean Duhamel
}

Institute of Polymer Research, Waterloo Institute of Nanotechnology, Department of Chemistry, University of Waterloo, Waterloo, ON N2L 3G1, Canada

* To Whom correspondence should be addressed. 


\section{EXPERIMENTAL}

Materials. Calf Thymus DNA (CT DNA), anhydrous copper sulfate, anhydrous nickel sulfate, and ethidium bromide (EB) were purchased from Sigma-Aldrich (Milwaukee, WI). Sodium sulfate and sodium chloride were purchased from Cambridge Isotope Laboratories, Inc. (Andover, MA) and EMD (Gibbstown, NJ), respectively. Ethidium bromide was recrystallized three times from 50:50 water:methanol to ensure its fluorescence purity. ${ }^{1}$ All solutions were prepared using doubly distilled water (deionized from Millipore Milli-RO 10 Plus and Milli-Q UF Plus (Bedford, MA)).

Solution Preparation. The electron transfer process between DNA-EB and copper or nickel cations was studied for at least five sodium sulfate concentrations of $5 \times 10^{-4}, 5 \times 10^{-3}, 1.25 \times 10^{-2}$, $2 \times 10^{-2}$, and $3.0 \times 10^{-2}$ mol. $\mathrm{L}^{-1}$ and three $\mathrm{NaCl}$ concentrations of $1 \times 10^{-2}, 2.5 \times 10^{-2}$, and $4.1 \times 10^{-2}$ mol. $\mathrm{L}^{-1}$. The ratio of metal to DNA phosphates was always smaller than 0.2 to minimize binding of the metal cations to the DNA bases. ${ }^{2}$ At least five CT DNA concentrations ranging from $0.02 \mathrm{wt} \%$ ( $0.30 \mathrm{mM}$ in base pair equivalent=bpe) to $0.09 \mathrm{wt} \%(1.36 \mathrm{mM}$ bpe $)$ were studied for each ionic strength. DNA stock solutions $(0.30 \mathrm{wt} \%$ or $4.5 \mathrm{mM}$ bpe $)$ were prepared by dissolving CT DNA overnight in water containing the appropriate sodium sulfate concentration. The absolute concentration of the DNA stock solution was obtained spectrophotometrically. Molar extinction coefficients per mole of bp of $\varepsilon_{260}=11,300 \mathrm{M}^{-1} \mathrm{~cm}^{-1}$ and $\varepsilon_{260}=10,400$ $\mathrm{M}^{-1} \cdot \mathrm{cm}^{-1}$ were determined experimentally for DNA in $5 \times 10^{-3} \mathrm{M}$ sodium sulfate and $1 \times 10^{-2} \mathrm{M}$ sodium chloride aqueous solution, respectively. Samples were freshly made on the day of use and all remaining CT DNA stock was discarded at the end of each day.

Steady-State Fluorescence Spectroscopy. Steady-state fluorescence measurements were carried out on a Photon Technology International (PTI) LS-100 steady-state fluorometer. The 
instrument was equipped with an Ushio UXL-75Xe Xenon arc flash lamp and a PTI 814 photomultiplier detection system using the right angle geometry. Samples were excited at 340 $\mathrm{nm}$ and the emission spectrum was collected from 450 to $650 \mathrm{~nm}$. The fluorescence intensity was taken as the integral under the fluorescence spectrum from $583 \mathrm{~nm}$ to $603 \mathrm{~nm}$ which spans the fluorescence maximum of DNA-intercalated ethidium bromide located near $590 \mathrm{~nm}$. A reference ethidium bromide solution in ethanol was used in order to account for deviations in the instrument lamp intensity throughout the day.

Time-Resolved Fluorescence Spectroscopy. Fluorescence decays were acquired with an IBH Ltd. time-resolved fluorometer equipped with an IBH $340 \mathrm{~nm}$ NanoLED. All solutions were excited at $340 \mathrm{~nm}$ and the emission was collected at $605 \mathrm{~nm}$. Decays were acquired using the right angle geometry and a filter with a cutoff of $570 \mathrm{~nm}$ to block potential light scattering from reaching the detection system. Fluorescence decays of the solutions were acquired over 1024 channels with a $1 \mathrm{MHz}$ repetition rate and a time per channel of $0.24 \mathrm{~ns} /$ channel. A Ludox solution was used to obtain the instrument response function (IRF). To ensure a high signal-tonoise ratio, the fluorescence decays and IRF were acquired until a peak maximum of 20,000 counts was reached. A least-squares analysis was used to fit the decays to the desired function and all decays were deconvoluted from the IRF profile.

Analysis of the Fluorescence Decays. The fluorescence decays of DNA-EB were first fit with Equation S1 using $n=2$ or 3 in the absence and presence of quencher, respectively. Equation S1 is a sum of exponentials that provides only qualitative information on the time scale over which quenching occurs. 


$$
i(t)=\sum_{i=1}^{n} a_{i} e^{-t / \tau_{i}}
$$

Quantitative information on how the excited ethidium bromide is being quenched via electron transfer to either copper or nickel is described by Equation S2 which is the Fluorescence Blob Model (FBM) equation. ${ }^{3-5}$ In Equation S2, the function $f(t)$ represents the natural decay of DNA-EB in the absence of quencher found to be biexponential and whose pre-exponential factors and decay times are fixed in the analysis. The resulting fits were characterized as "good" when the residuals and autocorrelation function of the residuals were randomly distributed around zero and the $\chi^{2}$ parameter was smaller than 1.3. A background and light scattering correction were applied to fit the fluorescence decays.

$$
\left[E B^{*}\right]=\left[E B^{*}\right]_{o}\left[f_{\text {slow }} \times f(t) \times \exp \left[-A_{2} t-A_{3}\left(1-\exp \left(-A_{4} t\right)\right)\right]+f_{\text {fast }} \exp \left(-t / \tau_{\text {fast }}\right)\right\rfloor
$$

The expressions of the parameters $A_{2}, A_{3}$, and $A_{4}$ used in Equation $\mathrm{S} 2$ are given in Equation $\mathrm{S} 3$.

$$
A_{2}=\langle n\rangle \frac{k_{\text {blob }} k_{e}[\text { blob }]}{k_{\text {blob }}+k_{e}[\text { blob }]} \quad A_{3}=\langle n\rangle \frac{k_{\text {blob }}^{2}}{\left(k_{\text {blob }}+k_{e}[\text { blob }]\right)^{2}} \quad A_{4}=k_{\text {blob }}+k_{e}[\text { blob }]
$$

$A_{2}, A_{3}$, and $A_{4}$ are functions of the three parameters $k_{\text {blob }}, k_{\mathrm{e}}[b l o b]$, and $<n>$ that describe the kinetics of electron transfer from DNA-EB to divalent metal cations. These three parameters are obtained from the fits of the fluorescence decays with the FBM equation (Equation S2). $k_{\text {blob }}$ is the rate constant of quenching of DNA-EB by one quencher inside the same blob, $k_{\mathrm{e}}[b l o b]$ is the product of the rate constant describing the exchange of quenchers between blobs $\left(k_{\mathrm{e}}\right)$ and the 
local blob concentration ([blob]), and $<n>$ is the average number of divalent metal cations per blob. An excited EB can be quenched quasi-instantaneously by nearby divalent metal cations with a decay time of $1-2 \mathrm{~ns}$ and this decay time is represented by the fraction $f_{\text {fast. }}$. The fraction $f_{\text {slow }}$ represents EB that is quenched with a decay time greater than $\tau_{\text {fast }}$ and can be handled by the FBM equation. All parameters retrieved from the analysis of our $745+$ fluorescence decays with Equations S1 and S2 are listed in Tables S1 - S10 in Supporting Information.

Blob Size and Binding Constant. The size of a blob $\left(N_{\mathrm{blob}}\right)$ and the binding constant $(K)$ of divalent metal cations to DNA are found through the FBM parameter $<n>$. The FBM parameter $<n>$ represents the average number of quenchers per blob and is expressed in Equation S4.

$$
<n>=\frac{\left[M^{2+}\right]_{\text {bound }}-\left[M^{2+}\right]_{o}}{[\text { blob }]}
$$

In Equation $\mathrm{S} 4,\left[\mathrm{M}^{2+}\right]_{0}$ is the onset quencher concentration representing the concentration of divalent metal cation required to induce a minimum amount of quenching that can be detected through our analysis and whose origin has been investigated earlier. ${ }^{5}\left[\mathrm{M}^{2+}\right]_{\text {bound }}$ represents the concentration of divalent metal cations bound to DNA. The equilibrium between free and DNAbound divalent metal cations is given in Equation S5. The equilibrium constant $K$ in Equation $\mathrm{S} 5$ can be used to derive the expression for $\left[\mathrm{M}^{2+}\right]_{\text {bound }}$ shown in Equation S6.

$$
\begin{gathered}
M_{\text {free }}^{2+}+D N A \stackrel{K}{\longleftarrow} M_{\text {bound }}^{2+} \\
{\left[M^{2+}\right]_{\text {bound }}=\frac{\left[M^{2+}\right]_{T}}{\frac{1}{K[D N A]}+1}}
\end{gathered}
$$


$\left[\mathrm{M}^{2+}\right]_{\mathrm{T}}$ in Equation $\mathrm{S} 6$ is the total divalent metal concentration and is equal to $\left[\mathrm{M}^{2+}\right]_{\text {free }}+$ $\left[\mathrm{M}^{2+}\right]_{\text {bound. }}$ Equation $\mathrm{S} 6$ can be used to determine the fraction of DNA negative charges that are neutralized by divalent metal cations which is readily obtained by taking the ratio $\left[\mathrm{M}^{2+}\right]_{\mathrm{b}} /[\mathrm{DNA}]$ where [DNA] is expressed in mol. $\mathrm{L}^{-1}$ of base pair. The blob concentration, [blob], in Equation S4 is equal to [DNA] $/ N_{\text {blob }}$ where $N_{\text {blob }}$ represents the number of bp constituting a blob. The expression of $\left[\mathrm{M}^{2+}\right]_{\text {bound }}$ in Equation S6 can be introduced into Equation S4 to yield Equation S7.

$$
\langle n\rangle=\frac{\left[M^{2+}\right]_{T}}{\frac{1}{K N_{\text {blob }}}+\frac{[D N A]}{N_{\text {blob }}}}-\frac{\left[M^{2+}\right]_{o} N_{\text {blob }}}{[D N A]}
$$

Equation $\mathrm{S} 7$ implies that a plot of $<n>$ versus $\left[\mathrm{M}^{2+}\right]_{\mathrm{T}}$ yields a straight line with a slope that depends on $N_{\text {blob }}, K$, and DNA concentration. A plot of the inverse of the slope of Equation S7 is expected to be linear with respect to DNA concentration as depicted in Equation S8.

$$
\frac{1}{\text { slope }}=\frac{1}{K N_{\text {blob }}}+\frac{[D N A]}{N_{\text {blob }}}
$$

Therefore, according to Equation S8, a plot of slope $e^{-1}$ versus [DNA] yields a straight line whose slope and intercept give $K$ and $N_{\text {blob, }}$, respectively. This procedure has been successfully validated in an earlier publication. ${ }^{5}$ 


\section{References}

1. Venner, H.; Zimmer, C. Biopolymers 1966, 4, 321-335.

2. Garland, F.; Graves, D. E.; Yielding, L. W.; Cheung, H. C. Biochemistry 1980, 19, 32213226.

3. Mathew, A.; Siu, H.; Duhamel, J. Macromolecules 1999, 32, 7100-7108.

4. Duhamel, J. Acc. Chem. Res. 2006, 39, 953-960.

5. Keyes Baig, C.; Duhamel, J. J. Phys. Chem. B 2010, 114, 13950-13960. 
Table 1a: Decay times and pre-exponential factors retrieved by using Equation $\mathrm{S} 1$ to fit the fluorescence decays of DNA-EB quenched by $\mathrm{Cu}^{2+}$ cations with an $\mathrm{Na}_{2} \mathrm{SO}_{4}$ concentration of $5 \times 10^{-4} \mathrm{M}$.

\begin{tabular}{|c|c|c|c|c|c|c|c|c|c|}
\hline $\begin{array}{c}{[\mathrm{DNA}]} \\
\mathrm{mM} \text { of } \mathrm{bp}\end{array}$ & {$\left[\mathrm{Cu}^{2+}\right](\mu \mathrm{M})$} & {$\left[\mathrm{Cu}^{2+}\right] /[\mathrm{P}]$} & $\begin{array}{r}\tau_{1} \\
\mathrm{~ns}\end{array}$ & $\begin{array}{r}\tau_{2} \\
\mathrm{~ns}\end{array}$ & $\begin{array}{r}\tau_{3} \\
\mathrm{~ns}\end{array}$ & $a_{1}$ & $a_{2}$ & $a_{3}$ & $\chi^{2}$ \\
\hline \multirow[t]{7}{*}{0.30} & 0 & 0.00 & 23.2 & 10.9 & & 0.95 & 0.05 & & 1.08 \\
\hline & 20 & 0.03 & 22.6 & 9.56 & & 0.85 & 0.15 & & 1.04 \\
\hline & 40 & 0.07 & 21.6 & 7.73 & & 0.76 & 0.24 & & 1.09 \\
\hline & 59 & 0.10 & 17.7 & 7.96 & 1.73 & 0.39 & 0.36 & 0.25 & 1.20 \\
\hline & 79 & 0.13 & 16.2 & 7.85 & 1.87 & 0.27 & 0.40 & 0.33 & 1.18 \\
\hline & 100 & 0.17 & 13.2 & 6.09 & 1.54 & 0.22 & 0.41 & 0.36 & 1.06 \\
\hline & 115 & 0.19 & 12.4 & 5.80 & 1.51 & 0.20 & 0.42 & 0.37 & 1.02 \\
\hline \multirow[t]{9}{*}{0.45} & 0 & 0.00 & 23.7 & 18.2 & & 0.81 & 0.19 & & 1.09 \\
\hline & 7 & 0.01 & 23.4 & 13.7 & & 0.87 & 0.13 & & 0.98 \\
\hline & 15 & 0.02 & 22.8 & 9.68 & & 0.89 & 0.11 & & 1.04 \\
\hline & 30 & 0.03 & 22.5 & 9.73 & & 0.83 & 0.17 & & 1.33 \\
\hline & 60 & 0.07 & 20.8 & 9.12 & 1.80 & 0.54 & 0.28 & 0.17 & 1.06 \\
\hline & 90 & 0.10 & 17.9 & 7.64 & 1.28 & 0.39 & 0.36 & 0.25 & 1.11 \\
\hline & 119 & 0.13 & 14.8 & 6.49 & 1.46 & 0.30 & 0.39 & 0.30 & 1.04 \\
\hline & 151 & 0.17 & 12.9 & 5.79 & 1.42 & 0.41 & 0.34 & 0.24 & 1.10 \\
\hline & 172 & 0.19 & 11.3 & 5.30 & 1.58 & 0.24 & 0.42 & 0.34 & 1.07 \\
\hline \multirow[t]{9}{*}{0.76} & 0 & 0.00 & 23.7 & 16.6 & & 0.85 & 0.15 & & 1.09 \\
\hline & 12 & 0.01 & 22.9 & 9.29 & & 0.93 & 0.07 & & 1.10 \\
\hline & 25 & 0.02 & 22.8 & 9.93 & & 0.90 & 0.10 & & 1.11 \\
\hline & 50 & 0.03 & 22.4 & 8.42 & & 0.85 & 0.15 & & 1.15 \\
\hline & 99 & 0.07 & 20.5 & 8.77 & 1.38 & 0.55 & 0.29 & 0.16 & 0.96 \\
\hline & 147 & 0.10 & 18.0 & 7.44 & 1.53 & 0.44 & 0.35 & 0.22 & 1.12 \\
\hline & 201 & 0.13 & 15.2 & 6.60 & 1.38 & 0.31 & 0.38 & 0.30 & 1.11 \\
\hline & 251 & 0.17 & 13.7 & 6.23 & 1.45 & 0.25 & 0.41 & 0.34 & 1.18 \\
\hline & 287 & 0.19 & 11.4 & 4.97 & 1.24 & 0.25 & 0.41 & 0.34 & 1.07 \\
\hline \multirow[t]{4}{*}{1.06} & 0 & 0.00 & 23.4 & 14.9 & & 0.90 & 0.10 & & 1.05 \\
\hline & 17 & 0.01 & 23.3 & 16.2 & & 0.86 & 0.14 & & 1.25 \\
\hline & 35 & 0.02 & 22.8 & 9.61 & & 0.90 & 0.10 & & 1.00 \\
\hline & 69 & 0.03 & 22.1 & 8.24 & & 0.80 & 0.20 & & 1.05 \\
\hline
\end{tabular}




\begin{tabular}{|c|c|c|c|c|c|c|c|c|c|}
\hline & 139 & 0.07 & 20.6 & 9.09 & 1.89 & 0.57 & 0.28 & 0.15 & 1.13 \\
\hline & 208 & 0.10 & 18.4 & 8.11 & 1.64 & 0.43 & 0.34 & 0.23 & 1.00 \\
\hline & 280 & 0.13 & 15.8 & 7.16 & 1.64 & 0.32 & 0.39 & 0.30 & 1.08 \\
\hline & 352 & 0.17 & 13.0 & 5.94 & 1.47 & 0.25 & 0.34 & 0.41 & 1.10 \\
\hline & 402 & 0.19 & 11.8 & 5.29 & 1.31 & 0.22 & 0.37 & 0.41 & 1.16 \\
\hline \multirow[t]{9}{*}{1.36} & 0 & 0.00 & 23.9 & 10.9 & & 0.95 & 0.05 & & 1.08 \\
\hline & 22 & 0.01 & 22.9 & 8.73 & & 0.94 & 0.06 & & 1.10 \\
\hline & 45 & 0.02 & 22.7 & 9.08 & & 0.88 & 0.12 & & 1.11 \\
\hline & 89 & 0.03 & 22.3 & 9.80 & 0.90 & 0.73 & 0.18 & 0.09 & 1.21 \\
\hline & 180 & 0.07 & 20.5 & 8.87 & 1.56 & 0.54 & 0.30 & 0.16 & 1.07 \\
\hline & 271 & 0.10 & 17.9 & 7.43 & 1.46 & 0.43 & 0.35 & 0.22 & 1.16 \\
\hline & 361 & 0.13 & 15.7 & 6.97 & 1.58 & 0.32 & 0.39 & 0.29 & 1.10 \\
\hline & 447 & 0.16 & 12.2 & 5.36 & 1.29 & 0.24 & 0.41 & 0.35 & 1.07 \\
\hline & 514 & 0.19 & 10.2 & 1.09 & 4.37 & 0.25 & 0.33 & 0.42 & 1.16 \\
\hline
\end{tabular}

Table 1b: Decay times and pre-exponential factors retrieved by using Equation $\mathrm{S} 1$ to fit the fluorescence decays of DNA-EB quenched by $\mathrm{Cu}^{2+}$ cations with an $\mathrm{Na}_{2} \mathrm{SO}_{4}$ concentration of $5 \times 10^{-3} \mathrm{M}$.

\begin{tabular}{|c|c|c|c|c|c|c|c|c|c|}
\hline $\begin{array}{c}{[\mathrm{DNA}]} \\
\mathrm{mM} \text { of bp }\end{array}$ & {$\left[\mathrm{Cu}^{2+}\right](\mu \mathrm{M})$} & {$\left[\mathrm{Cu}^{2+}\right] /[\mathrm{P}]$} & $\begin{array}{c}\tau_{1} \\
\mathrm{~ns}\end{array}$ & $\begin{array}{r}\tau_{2} \\
\mathrm{~ns}\end{array}$ & $\begin{array}{r}\tau_{3} \\
\mathrm{~ns}\end{array}$ & $a_{1}$ & $a_{2}$ & $a_{3}$ & $\chi^{2}$ \\
\hline \multirow[t]{7}{*}{0.30} & 0 & 0.00 & 22.9 & 9.54 & & 0.96 & 0.04 & & 1.06 \\
\hline & 20 & 0.03 & 22.5 & 8.48 & & 0.87 & 0.13 & & 1.08 \\
\hline & 40 & 0.07 & 20.7 & 8.00 & 0.75 & 0.58 & 0.24 & 0.17 & 1.09 \\
\hline & 60 & 0.10 & 19.3 & 8.50 & 1.59 & 0.46 & 0.33 & 0.21 & 1.18 \\
\hline & 79 & 0.13 & 17.5 & 7.24 & 1.20 & 0.38 & 0.36 & 0.25 & 1.15 \\
\hline & 99 & 0.16 & 16.2 & 7.47 & 1.78 & 0.32 & 0.38 & 0.29 & 1.20 \\
\hline & 115 & 0.19 & 14.9 & 6.66 & 1.51 & 0.29 & 0.40 & 0.31 & 1.19 \\
\hline \multirow[t]{6}{*}{0.45} & 0 & 0.00 & 23.8 & 17.2 & & 0.84 & 0.16 & & 0.99 \\
\hline & 8 & 0.01 & 23.0 & 9.59 & & 0.93 & 0.07 & & 1.05 \\
\hline & 15 & 0.02 & 22.8 & 8.09 & & 0.91 & 0.09 & & 1.03 \\
\hline & 30 & 0.03 & 22.5 & 7.86 & & 0.85 & 0.15 & & 1.02 \\
\hline & 59 & 0.06 & 20.7 & 9.16 & 1.82 & 0.59 & 0.26 & 0.15 & 1.08 \\
\hline & 90 & 0.10 & 19.0 & 8.22 & 1.68 & 0.46 & 0.33 & 0.21 & 1.07 \\
\hline
\end{tabular}




\begin{tabular}{|c|c|c|c|c|c|c|c|c|c|}
\hline & 120 & 0.13 & 16.8 & 7.60 & 1.74 & 0.36 & 0.36 & 0.28 & 1.10 \\
\hline & 149 & 0.16 & 15.1 & 6.87 & 1.68 & 0.29 & 0.40 & 0.31 & 1.21 \\
\hline & 172 & 0.19 & 14.0 & 6.40 & 1.64 & 0.26 & 0.40 & 0.34 & 1.15 \\
\hline \multirow[t]{9}{*}{0.76} & 0 & 0.00 & 24.0 & 17.9 & & 0.81 & 0.19 & & 1.17 \\
\hline & 13 & 0.01 & 23.5 & 15.6 & & 0.86 & 0.14 & & 1.13 \\
\hline & 25 & 0.02 & 23.1 & 10.2 & & 0.91 & 0.09 & & 1.06 \\
\hline & 50 & 0.03 & 22.2 & 8.80 & 1.35 & 0.78 & 0.15 & 0.06 & 1.18 \\
\hline & 100 & 0.07 & 21.0 & 9.30 & 1.80 & 0.61 & 0.25 & 0.14 & 1.07 \\
\hline & 148 & 0.10 & 20.0 & 9.50 & 2.08 & 0.45 & 0.34 & 0.21 & 0.98 \\
\hline & 200 & 0.13 & 17.3 & 7.57 & 1.53 & 0.36 & 0.37 & 0.28 & 1.10 \\
\hline & 249 & 0.16 & 15.8 & 7.64 & 1.89 & 0.26 & 0.40 & 0.33 & 1.06 \\
\hline & 287 & 0.19 & 14.4 & 6.60 & 1.70 & 0.27 & 0.39 & 0.33 & 1.15 \\
\hline \multirow[t]{9}{*}{1.06} & 0 & 0.00 & 24.1 & 17.8 & & 0.81 & 0.19 & & 1.11 \\
\hline & 17 & 0.01 & 23.3 & 11.1 & & 0.91 & 0.09 & & 1.25 \\
\hline & 35 & 0.02 & 22.9 & 8.95 & & 0.88 & 0.12 & & 1.23 \\
\hline & 69 & 0.03 & 22.3 & 7.95 & & 0.81 & 0.19 & & 1.12 \\
\hline & 138 & 0.07 & 21.3 & 10.1 & 2.55 & 0.59 & 0.27 & 0.14 & 1.15 \\
\hline & 208 & 0.10 & 19.3 & 8.13 & 1.41 & 0.46 & 0.34 & 0.2 & 1.27 \\
\hline & 280 & 0.13 & 16.9 & 7.77 & 1.78 & 0.32 & 0.39 & 0.29 & 1.12 \\
\hline & 347 & 0.16 & 13.5 & 6.09 & 1.58 & 0.26 & 0.39 & 0.35 & 1.22 \\
\hline & 399 & 0.19 & 11.7 & 5.21 & 1.36 & 0.24 & 0.41 & 0.35 & 1.32 \\
\hline \multirow[t]{9}{*}{1.36} & 0 & 0.00 & 23.7 & 9.47 & & 0.95 & 0.05 & & 1.17 \\
\hline & 22 & 0.01 & 23.3 & 12.9 & & 0.89 & 0.11 & & 1.11 \\
\hline & 45 & 0.02 & 22.7 & 8.42 & & 0.89 & 0.11 & & 1.13 \\
\hline & 90 & 0.03 & 22.4 & 9.67 & 1.25 & 0.75 & 0.17 & 0.08 & 1.05 \\
\hline & 179 & 0.07 & 21.1 & 8.61 & 1.52 & 0.61 & 0.26 & 0.13 & 1.05 \\
\hline & 269 & 0.10 & 19.3 & 8.03 & 1.34 & 0.46 & 0.33 & 0.21 & 1.05 \\
\hline & 349 & 0.13 & 16.6 & 7.68 & 1.79 & 0.32 & 0.37 & 0.31 & 1.05 \\
\hline & 455 & 0.17 & 14.3 & 6.55 & 1.58 & 0.26 & 0.40 & 0.34 & 1.12 \\
\hline & 505 & 0.19 & 12.9 & 6.28 & 1.77 & 0.23 & 0.40 & 0.37 & 1.11 \\
\hline
\end{tabular}


Table 1c: Decay times and pre-exponential factors retrieved by using Equation $\mathrm{S} 1$ to fit the fluorescence decays of DNA-EB quenched by $\mathrm{Cu}^{2+}$ cations with an $\mathrm{Na}_{2} \mathrm{SO}_{4}$ concentration of $7.5 \times 10^{-3} \mathrm{M}$.

\begin{tabular}{|c|c|c|c|c|c|c|c|c|c|}
\hline $\begin{array}{c}\text { [DNA] } \\
\mathrm{mM} \text { of bp }\end{array}$ & {$\left[\mathrm{Cu}^{2+}\right](\mu \mathrm{M})$} & {$\left[\mathrm{Cu}^{2+}\right] /[\mathrm{P}]$} & $\begin{array}{c}\tau_{1} \\
\mathrm{~ns}\end{array}$ & $\begin{array}{r}\tau_{2} \\
\mathrm{~ns}\end{array}$ & $\begin{array}{r}\tau_{3} \\
\mathrm{~ns}\end{array}$ & $a_{1}$ & $a_{2}$ & $a_{3}$ & $\chi^{2}$ \\
\hline \multirow{10}{*}{0.30} & 0 & 0.00 & 22.6 & 13.0 & & 0.93 & 0.07 & & 1.00 \\
\hline & 10 & 0.02 & 22.6 & 16.8 & 4.95 & 0.67 & 0.24 & 0.08 & 1.03 \\
\hline & 22 & 0.04 & 21.5 & 12.8 & 2.83 & 0.64 & 0.24 & 0.12 & 1.21 \\
\hline & 31 & 0.05 & 20.4 & 9.58 & 1.72 & 0.61 & 0.25 & 0.14 & 1.15 \\
\hline & 40 & 0.07 & 19.6 & 8.08 & 1.35 & 0.57 & 0.28 & 0.15 & 1.07 \\
\hline & 54 & 0.09 & 18.6 & 8.63 & 1.76 & 0.43 & 0.34 & 0.23 & 0.98 \\
\hline & 60 & 0.10 & 18.6 & 8.47 & 1.46 & 0.42 & 0.34 & 0.24 & 1.26 \\
\hline & 77 & 0.13 & 18.2 & 8.67 & 1.94 & 0.39 & 0.36 & 0.25 & 0.98 \\
\hline & 101 & 0.17 & 16.2 & 7.13 & 1.36 & 0.30 & 0.40 & 0.30 & 1.45 \\
\hline & 112 & 0.19 & 15.8 & 7.48 & 2.03 & 0.30 & 0.40 & 0.30 & 1.11 \\
\hline \multirow[t]{10}{*}{0.45} & 0 & 0.00 & 22.8 & 11.0 & & 0.95 & 0.04 & & 0.99 \\
\hline & 16 & 0.01 & 22.9 & 20.9 & 7.84 & 0.49 & 0.40 & 0.11 & 1.17 \\
\hline & 30 & 0.03 & 22.4 & 14.1 & 3.70 & 0.65 & 0.24 & 0.11 & 1.00 \\
\hline & 48 & 0.02 & 21.2 & 11.0 & 2.81 & 0.61 & 0.25 & 0.14 & 1.12 \\
\hline & 62 & 0.07 & 20.3 & 8.78 & 1.71 & 0.59 & 0.27 & 0.14 & 1.11 \\
\hline & 79 & 0.03 & 19.7 & 8.66 & 1.75 & 0.52 & 0.30 & 0.18 & 0.93 \\
\hline & 89 & 0.10 & 19.2 & 8.30 & 1.44 & 0.47 & 0.31 & 0.22 & 1.10 \\
\hline & 118 & 0.05 & 18.1 & 8.32 & 1.73 & 0.38 & 0.36 & 0.26 & 1.17 \\
\hline & 155 & 0.17 & 16.1 & 7.15 & 1.81 & 0.33 & 0.39 & 0.28 & 1.21 \\
\hline & 171 & 0.19 & 15.5 & 7.03 & 1.59 & 0.29 & 0.38 & 0.33 & 1.17 \\
\hline \multirow[t]{9}{*}{0.76} & 0 & 0.00 & 23.1 & 11.6 & & 0.93 & 0.07 & & 1.14 \\
\hline & 27 & 0.02 & 22.6 & 9.86 & & 0.87 & 0.13 & & 1.27 \\
\hline & 50 & 0.03 & 23.0 & 16.6 & 5.30 & 0.58 & 0.29 & 0.13 & 1.21 \\
\hline & 78 & 0.05 & 21.2 & 8.33 & 0.75 & 0.62 & 0.23 & 0.15 & 1.20 \\
\hline & 99 & 0.07 & 20.7 & 9.79 & 1.97 & 0.56 & 0.27 & 0.17 & 1.09 \\
\hline & 134 & 0.09 & 19.6 & 8.46 & 1.39 & 0.47 & 0.33 & 0.20 & 1.08 \\
\hline & 154 & 0.10 & 19.3 & 8.96 & 1.83 & 0.43 & 0.35 & 0.22 & 1.06 \\
\hline & 195 & 0.13 & 17.1 & 7.24 & 1.51 & 0.40 & 0.36 & 0.25 & 1.08 \\
\hline & 257 & 0.17 & 15.4 & 7.20 & 1.92 & 0.27 & 0.40 & 0.33 & 0.99 \\
\hline
\end{tabular}




\begin{tabular}{|c|c|c|c|c|c|c|c|c|c|}
\hline & 280 & 0.19 & 14.4 & 6.20 & 1.33 & 0.27 & 0.41 & 0.31 & 1.23 \\
\hline \multirow[t]{10}{*}{1.06} & 0 & 0.00 & 23.1 & 11.3 & & 0.95 & 0.05 & & 1.22 \\
\hline & 36 & 0.02 & 23.9 & 16.9 & 3.89 & 0.64 & 0.29 & 0.07 & 1.16 \\
\hline & 72 & 0.03 & 20.3 & 23.1 & 6.74 & 0.30 & 0.56 & 0.14 & 1.13 \\
\hline & 110 & 0.05 & 21.7 & 10.2 & 2.02 & 0.64 & 0.25 & 0.12 & 1.13 \\
\hline & 141 & 0.07 & 21.4 & 10.1 & 1.63 & 0.57 & 0.28 & 0.14 & 1.11 \\
\hline & 187 & 0.09 & 20.0 & 8.66 & 1.53 & 0.50 & 0.32 & 0.18 & 1.15 \\
\hline & 211 & 0.10 & 19.1 & 8.61 & 2.00 & 0.45 & 0.34 & 0.21 & 1.12 \\
\hline & 274 & 0.13 & 17.6 & 7.92 & 1.65 & 0.35 & 0.38 & 0.27 & 1.07 \\
\hline & 352 & 0.17 & 15.2 & 7.08 & 1.77 & 0.27 & 0.41 & 0.32 & 1.17 \\
\hline & 392 & 0.18 & 14.1 & 6.50 & 1.75 & 0.27 & 0.41 & 0.32 & 1.33 \\
\hline \multirow[t]{10}{*}{1.36} & 0 & 0.00 & 23.2 & 11.0 & & 0.94 & 0.06 & & 1.04 \\
\hline & 44 & 0.02 & 23.6 & 21.2 & 7.63 & 0.11 & 0.39 & 0.50 & 1.20 \\
\hline & 89 & 0.03 & 22.3 & 9.88 & 1.23 & 0.74 & 0.17 & 0.09 & 1.23 \\
\hline & 138 & 0.05 & 21.8 & 9.84 & 1.73 & 0.66 & 0.23 & 0.11 & 1.12 \\
\hline & 180 & 0.07 & 21.4 & 10.6 & 2.38 & 0.55 & 0.30 & 0.15 & 1.08 \\
\hline & 243 & 0.09 & 19.8 & 8.41 & 1.55 & 0.50 & 0.31 & 0.19 & 1.28 \\
\hline & 272 & 0.10 & 19.6 & 9.32 & 1.97 & 0.44 & 0.34 & 0.22 & 1.19 \\
\hline & 354 & 0.13 & 17.2 & 7.30 & 1.68 & 0.39 & 0.37 & 0.24 & 1.28 \\
\hline & 455 & 0.17 & 14.7 & 6.78 & 1.66 & 0.28 & 0.39 & 0.33 & 1.10 \\
\hline & 505 & 0.19 & 13.3 & 5.62 & 1.20 & 0.28 & 0.38 & 0.34 & 1.22 \\
\hline
\end{tabular}

Table 1d: Decay times and pre-exponential factors retrieved by using Equation $\mathrm{S} 1$ to fit the fluorescence decays of DNA-EB quenched by $\mathrm{Cu}^{2+}$ cations with an $\mathrm{Na}_{2} \mathrm{SO}_{4}$ concentration of $1.25 \times 10^{-2} \mathrm{M}$.

\begin{tabular}{|c|c|c|c|c|c|c|c|c|c|}
\hline $\begin{array}{c}{[\mathrm{DNA}]} \\
\mathrm{mM} \text { of } \mathrm{bp}\end{array}$ & {$\left[\mathrm{Cu}^{2+}\right](\mu \mathrm{M})$} & {$\left[\mathrm{Cu}^{2+}\right] /[\mathrm{P}]$} & $\begin{array}{r}\tau_{1} \\
\mathrm{~ns}\end{array}$ & $\begin{array}{r}\tau_{2} \\
\mathrm{~ns}\end{array}$ & $\begin{array}{r}\tau_{3} \\
\mathrm{~ns}\end{array}$ & $a_{1}$ & $a_{2}$ & $a_{3}$ & $\chi^{2}$ \\
\hline \multirow[t]{6}{*}{0.30} & 0 & 0.00 & 22.5 & 12.0 & & 0.95 & 0.05 & & 0.97 \\
\hline & 10 & 0.02 & 21.7 & 7.21 & & 0.87 & 0.13 & & 1.02 \\
\hline & 22 & 0.04 & 21.1 & 8.96 & 1.60 & 0.74 & 0.19 & 0.08 & 1.24 \\
\hline & 31 & 0.05 & 20.5 & 8.95 & 1.36 & 0.64 & 0.23 & 0.13 & 1.04 \\
\hline & 40 & 0.07 & 20.3 & 8.87 & 1.23 & 0.59 & 0.26 & 0.15 & 1.10 \\
\hline & 54 & 0.09 & 19.4 & 8.50 & 1.63 & 0.54 & 0.29 & 0.18 & 1.20 \\
\hline
\end{tabular}




\begin{tabular}{|c|c|c|c|c|c|c|c|c|c|}
\hline & 60 & 0.10 & 19.1 & 8.67 & 1.97 & 0.51 & 0.30 & 0.19 & 1.27 \\
\hline & 77 & 0.13 & 18.6 & 8.40 & 1.66 & 0.45 & 0.34 & 0.21 & 1.25 \\
\hline & 101 & 0.17 & 17.3 & 7.18 & 1.35 & 0.42 & 0.34 & 0.24 & 1.18 \\
\hline & 112 & 0.19 & 17.2 & 7.43 & 1.38 & 0.38 & 0.37 & 0.25 & 1.30 \\
\hline \multirow[t]{10}{*}{0.45} & 0 & 0.00 & 22.8 & 9.51 & & 0.95 & 0.05 & & 1.12 \\
\hline & 15 & 0.02 & 23.3 & 19.5 & 7.67 & 0.55 & 0.36 & 0.09 & 1.10 \\
\hline & 32 & 0.04 & 22.2 & 12.9 & 2.66 & 0.67 & 0.23 & 0.10 & 1.17 \\
\hline & 46 & 0.05 & 20.8 & 7.78 & 0.79 & 0.65 & 0.23 & 0.13 & 1.28 \\
\hline & 58 & 0.06 & 20.6 & 8.76 & 1.37 & 0.60 & 0.27 & 0.14 & 1.26 \\
\hline & 80 & 0.09 & 19.7 & 8.83 & 1.93 & 0.52 & 0.31 & 0.17 & 1.08 \\
\hline & 90 & 0.10 & 19.7 & 9.71 & 1.96 & 0.47 & 0.32 & 0.21 & 1.16 \\
\hline & 118 & 0.13 & 18.5 & 8.36 & 1.82 & 0.43 & 0.34 & 0.22 & 1.11 \\
\hline & 153 & 0.17 & 17.5 & 8.06 & 1.77 & 0.36 & 0.38 & 0.27 & 1.21 \\
\hline & 166 & 0.18 & 16.7 & 7.21 & 1.56 & 0.36 & 0.36 & 0.28 & 1.14 \\
\hline \multirow[t]{10}{*}{0.76} & 0 & 0.00 & 23.2 & 12.0 & & 0.94 & 0.06 & & 1.03 \\
\hline & 29 & 0.02 & 22.8 & 22.4 & 8.84 & 0.47 & 0.39 & 0.14 & 1.04 \\
\hline & 51 & 0.03 & 22.7 & 16.2 & 5.04 & 0.65 & 0.22 & 0.13 & 1.15 \\
\hline & 77 & 0.05 & 21.9 & 10.3 & 1.74 & 0.66 & 0.22 & 0.11 & 1.00 \\
\hline & 99 & 0.07 & 21.3 & 9.46 & 1.79 & 0.61 & 0.27 & 0.12 & 1.14 \\
\hline & 135 & 0.09 & 20.3 & 9.02 & 1.64 & 0.52 & 0.30 & 0.18 & 1.06 \\
\hline & 148 & 0.10 & 19.9 & 8.82 & 1.72 & 0.50 & 0.31 & 0.19 & 1.03 \\
\hline & 192 & 0.13 & 18.8 & 8.20 & 1.64 & 0.43 & 0.35 & 0.22 & 1.10 \\
\hline & 252 & 0.17 & 17.5 & 8.16 & 1.81 & 0.34 & 0.37 & 0.29 & 1.19 \\
\hline & 278 & 0.18 & 16.4 & 7.62 & 1.86 & 0.33 & 0.38 & 0.29 & 1.27 \\
\hline \multirow[t]{10}{*}{1.06} & 0 & 0.00 & 23.3 & 12.2 & & 0.94 & 0.07 & & 1.09 \\
\hline & 36 & 0.02 & 24.1 & 19.3 & 4.97 & 0.52 & 0.40 & 0.08 & 1.15 \\
\hline & 70 & 0.03 & 22.4 & 10.6 & 1.64 & 0.73 & 0.18 & 0.09 & 1.13 \\
\hline & 110 & 0.05 & 21.6 & 9.77 & 1.26 & 0.61 & 0.26 & 0.13 & 1.11 \\
\hline & 138 & 0.07 & 21.2 & 9.96 & 2.11 & 0.59 & 0.27 & 0.14 & 1.11 \\
\hline & 185 & 0.09 & 20.0 & 8.58 & 1.36 & 0.49 & 0.33 & 0.18 & 1.15 \\
\hline & 210 & 0.10 & 19.4 & 8.95 & 2.06 & 0.46 & 0.33 & 0.21 & 1.12 \\
\hline & 274 & 0.13 & 18.0 & 8.17 & 1.69 & 0.38 & 0.37 & 0.25 & 1.25 \\
\hline & 356 & 0.17 & 16.0 & 6.90 & 1.39 & 0.31 & 0.39 & 0.30 & 1.24 \\
\hline & 391 & 0.18 & 15.6 & 7.08 & 1.53 & 0.28 & 0.41 & 0.31 & 1.26 \\
\hline
\end{tabular}




\begin{tabular}{|c|c|c|c|c|c|c|c|c|c|}
\hline \multirow[t]{10}{*}{1.36} & 0 & 0.00 & 23.2 & 9.81 & & 0.94 & 0.06 & & 1.14 \\
\hline & 43 & 0.02 & 23.1 & 22.6 & 9.02 & 0.48 & 0.40 & 0.12 & 1.06 \\
\hline & 89 & 0.03 & 23.2 & 14.5 & 4.12 & 0.67 & 0.25 & 0.09 & 1.02 \\
\hline & 139 & 0.05 & 21.9 & 10.0 & 1.73 & 0.66 & 0.23 & 0.11 & 1.16 \\
\hline & 179 & 0.07 & 21.6 & 9.97 & 1.88 & 0.61 & 0.26 & 0.13 & 1.17 \\
\hline & 243 & 0.09 & 20.4 & 9.40 & 1.86 & 0.51 & 0.30 & 0.19 & 1.01 \\
\hline & 271 & 0.10 & 19.9 & 8.91 & 1.73 & 0.49 & 0.32 & 0.20 & 1.04 \\
\hline & 349 & 0.13 & 18.4 & 7.95 & 1.29 & 0.39 & 0.36 & 0.25 & 1.21 \\
\hline & 455 & 0.17 & 16.2 & 6.91 & 1.31 & 0.33 & 0.37 & 0.29 & 1.20 \\
\hline & 503 & 0.18 & 15.3 & 6.66 & 1.51 & 0.32 & 0.38 & 0.30 & 1.19 \\
\hline
\end{tabular}

Table 1e: Decay times and pre-exponential factors retrieved by using Equation S1 to fit the fluorescence decays of DNA-EB quenched by $\mathrm{Cu}^{2+}$ cations with an $\mathrm{Na}_{2} \mathrm{SO}_{4}$ concentration of $2.1 \times 10^{-2} \mathrm{M}$.

\begin{tabular}{|c|c|c|c|c|c|c|c|c|c|}
\hline $\begin{array}{c}\text { [DNA] } \\
\text { mM of bp }\end{array}$ & {$\left[\mathrm{Cu}^{2+}\right](\mu \mathrm{M})$} & {$\left[\mathrm{Cu}^{2+}\right] /[\mathrm{P}]$} & $\begin{array}{r}\tau_{1} \\
\mathrm{~ns}\end{array}$ & $\begin{array}{r}\tau_{2} \\
\mathrm{~ns}\end{array}$ & $\begin{array}{r}\tau_{3} \\
\mathrm{~ns}\end{array}$ & $a_{1}$ & $a_{2}$ & $a_{3}$ & $\chi^{2}$ \\
\hline \multirow[t]{10}{*}{0.30} & 0 & 0.00 & 22.4 & 8.11 & & 0.97 & 0.03 & & 1.01 \\
\hline & 11 & 0.02 & 21.8 & 7.22 & & 0.89 & 0.10 & & 1.06 \\
\hline & 20 & 0.03 & 21.8 & 11.3 & 2.04 & 0.74 & 0.18 & 0.08 & 0.98 \\
\hline & 32 & 0.05 & 21.0 & 8.92 & 1.39 & 0.73 & 0.19 & 0.08 & 1.11 \\
\hline & 39 & 0.06 & 21.9 & 15.1 & 3.24 & 0.51 & 0.33 & 0.16 & 1.19 \\
\hline & 54 & 0.09 & 20.6 & 9.65 & 1.73 & 0.61 & 0.25 & 0.14 & 1.04 \\
\hline & 61 & 0.10 & 20.1 & 8.81 & 1.65 & 0.62 & 0.23 & 0.15 & 1.05 \\
\hline & 77 & 0.13 & 19.7 & 8.58 & 1.63 & 0.57 & 0.27 & 0.16 & 1.27 \\
\hline & 102 & 0.17 & 19.3 & 8.83 & 1.91 & 0.52 & 0.30 & 0.19 & 1.06 \\
\hline & 111 & 0.18 & 18.9 & 7.91 & 1.11 & 0.48 & 0.31 & 0.20 & 1.08 \\
\hline \multirow[t]{7}{*}{0.45} & 0 & 0.00 & 23.3 & 13.3 & & 0.92 & 0.08 & & 0.98 \\
\hline & 15 & 0.02 & 23.0 & 10.5 & & 0.90 & 0.10 & & 1.07 \\
\hline & 30 & 0.03 & 22.5 & 8.29 & & 0.84 & 0.16 & & 1.05 \\
\hline & 59 & 0.06 & 22.1 & 10.6 & 1.28 & 0.66 & 0.22 & 0.12 & 1.05 \\
\hline & 89 & 0.10 & 21.3 & 9.27 & 1.71 & 0.63 & 0.25 & 0.12 & 1.08 \\
\hline & 121 & 0.13 & 20.6 & 8.43 & 1.22 & 0.56 & 0.28 & 0.16 & 1.10 \\
\hline & 151 & 0.17 & 20.3 & 9.36 & 1.71 & 0.50 & 0.32 & 0.18 & 1.10 \\
\hline
\end{tabular}




\begin{tabular}{|c|c|c|c|c|c|c|c|c|c|}
\hline & 173 & 0.19 & 19.6 & 9.03 & 1.89 & 0.48 & 0.33 & 0.19 & 1.07 \\
\hline \multirow[t]{9}{*}{0.76} & 0 & 0.00 & 23.1 & 10.3 & & 0.96 & 0.04 & & 1.10 \\
\hline & 12 & 0.01 & 23.2 & 10.5 & & 0.92 & 0.08 & & 1.11 \\
\hline & 25 & 0.02 & 22.9 & 9.99 & & 0.91 & 0.09 & & 1.08 \\
\hline & 50 & 0.03 & 22.9 & 5.27 & & 0.95 & 0.05 & & 1.10 \\
\hline & 101 & 0.07 & 21.9 & 8.84 & 1.38 & 0.72 & 0.19 & 0.09 & 1.12 \\
\hline & 150 & 0.10 & 21.4 & 9.87 & 2.11 & 0.61 & 0.26 & 0.13 & 1.14 \\
\hline & 199 & 0.13 & 20.0 & 8.84 & 1.95 & 0.54 & 0.30 & 0.16 & 1.24 \\
\hline & 245 & 0.16 & 19.4 & 8.90 & 1.81 & 0.45 & 0.33 & 0.22 & 1.14 \\
\hline & 292 & 0.19 & 18.1 & 7.62 & 1.38 & 0.42 & 0.34 & 0.24 & 1.29 \\
\hline \multirow[t]{9}{*}{1.06} & 0 & 0.00 & 23.4 & 13.4 & & 0.91 & 0.09 & & 1.08 \\
\hline & 18 & 0.01 & 23.0 & 9.29 & & 0.92 & 0.08 & & 1.12 \\
\hline & 35 & 0.02 & 22.6 & 7.94 & & 0.91 & 0.09 & & 1.16 \\
\hline & 69 & 0.03 & 22.4 & 8.19 & & 0.83 & 0.17 & & 1.17 \\
\hline & 140 & 0.07 & 21.7 & 9.43 & 1.18 & 0.64 & 0.23 & 0.13 & 1.03 \\
\hline & 211 & 0.10 & 20.2 & 8.44 & 1.51 & 0.56 & 0.28 & 0.16 & 1.10 \\
\hline & 282 & 0.13 & 19.0 & 8.39 & 1.61 & 0.46 & 0.32 & 0.22 & 0.95 \\
\hline & 345 & 0.16 & 17.9 & 7.71 & 1.62 & 0.43 & 0.35 & 0.23 & 1.03 \\
\hline & 397 & 0.19 & 17.0 & 7.19 & 1.51 & 0.40 & 0.36 & 0.24 & 1.08 \\
\hline \multirow[t]{9}{*}{1.36} & 0 & 0.00 & 23.3 & 13.2 & & 0.93 & 0.07 & & 1.00 \\
\hline & 22 & 0.01 & 23.1 & 10.8 & & 0.93 & 0.07 & & 1.09 \\
\hline & 45 & 0.02 & 22.7 & 7.01 & & 0.92 & 0.08 & & 0.96 \\
\hline & 88 & 0.03 & 22.7 & 8.53 & & 0.85 & 0.15 & & 1.10 \\
\hline & 176 & 0.06 & 22.0 & 9.64 & 1.66 & 0.68 & 0.22 & 0.10 & 1.10 \\
\hline & 268 & 0.10 & 20.8 & 8.84 & 1.48 & 0.58 & 0.27 & 0.15 & 1.19 \\
\hline & 366 & 0.13 & 19.6 & 8.92 & 1.67 & 0.46 & 0.33 & 0.21 & 0.99 \\
\hline & 449 & 0.16 & 18.3 & 8.37 & 1.84 & 0.40 & 0.36 & 0.24 & 1.18 \\
\hline & 522 & 0.19 & 16.7 & 7.21 & 1.48 & 0.40 & 0.34 & 0.26 & 1.17 \\
\hline
\end{tabular}


Table 1f: Decay times and pre-exponential factors retrieved from by using Equation S1 to fit the fluorescence decays of DNA-EB quenched by $\mathrm{Cu}^{2+}$ cations with an $\mathrm{Na}_{2} \mathrm{SO}_{4}$ concentration of $3 \times 10^{-2} \mathrm{M}$.

\begin{tabular}{|c|c|c|c|c|c|c|c|c|c|}
\hline $\begin{array}{c}{[\mathrm{DNA}]} \\
\mathrm{mM} \text { of bp }\end{array}$ & {$\left[\mathrm{Cu}^{2+}\right](\mu \mathrm{M})$} & {$\left[\mathrm{Cu}^{2+}\right] /[\mathrm{P}]$} & $\begin{array}{r}\tau_{1} \\
\mathrm{~ns}\end{array}$ & $\begin{array}{c}\tau_{2} \\
\mathrm{~ns}\end{array}$ & $\begin{array}{r}\tau_{3} \\
\mathrm{~ns}\end{array}$ & $a_{1}$ & $a_{2}$ & $a_{3}$ & $\chi^{2}$ \\
\hline \multirow[t]{9}{*}{0.30} & 0 & 0.00 & 23.7 & 15.3 & & 0.88 & 0.12 & & 1.13 \\
\hline & 21 & 0.03 & 23.3 & 16.0 & 5.15 & 0.69 & 0.22 & 0.08 & 1.17 \\
\hline & 32 & 0.05 & 22.5 & 9.80 & 0.83 & 0.72 & 0.17 & 0.11 & 1.14 \\
\hline & 41 & 0.07 & 22.2 & 9.98 & 1.64 & 0.72 & 0.20 & 0.08 & 1.08 \\
\hline & 54 & 0.09 & 22.4 & 12.1 & 2.45 & 0.64 & 0.24 & 0.12 & 1.09 \\
\hline & 59 & 0.10 & 22.0 & 11.2 & 1.95 & 0.66 & 0.23 & 0.11 & 1.01 \\
\hline & 81 & 0.13 & 22.0 & 11.0 & 2.11 & 0.60 & 0.26 & 0.14 & 1.03 \\
\hline & 101 & 0.17 & 21.5 & 11.1 & 2.47 & 0.55 & 0.29 & 0.16 & 1.10 \\
\hline & 113 & 0.19 & 20.9 & 9.19 & 1.63 & 0.59 & 0.25 & 0.16 & 1.11 \\
\hline \multirow[t]{10}{*}{0.45} & 0 & 0.00 & 23.9 & 16.2 & & 0.84 & 0.16 & & 1.21 \\
\hline & 25 & 0.02 & 23.2 & 12.5 & 1.54 & 0.80 & 0.13 & 0.07 & 1.12 \\
\hline & 50 & 0.03 & 23.0 & 11.3 & 1.01 & 0.74 & 0.19 & 0.08 & 1.10 \\
\hline & 80 & 0.05 & 22.7 & 11.9 & 2.11 & 0.70 & 0.22 & 0.10 & 1.06 \\
\hline & 103 & 0.07 & 22.1 & 9.13 & 0.81 & 0.68 & 0.21 & 0.11 & 1.11 \\
\hline & 138 & 0.09 & 22.0 & 10.9 & 1.98 & 0.62 & 0.25 & 0.13 & 1.17 \\
\hline & 150 & 0.10 & 21.9 & 11.1 & 1.96 & 0.60 & 0.26 & 0.15 & 1.00 \\
\hline & 196 & 0.13 & 21.2 & 9.19 & 1.23 & 0.58 & 0.27 & 0.15 & 1.01 \\
\hline & 254 & 0.17 & 20.9 & 10.3 & 1.97 & 0.52 & 0.30 & 0.18 & 1.09 \\
\hline & 281 & 0.19 & 20.5 & 9.90 & 2.04 & 0.51 & 0.30 & 0.19 & 1.12 \\
\hline \multirow[t]{5}{*}{0.61} & 0 & 0.00 & 24.2 & 16.4 & & 0.80 & 0.20 & & 1.11 \\
\hline & 19 & 0.02 & 23.3 & 12.1 & 0.40 & 0.77 & 0.14 & 0.09 & 1.17 \\
\hline & 41 & 0.03 & 22.8 & 10.9 & 1.10 & 0.75 & 0.17 & 0.08 & 1.18 \\
\hline & 63 & 0.05 & 23.2 & 13.5 & 2.41 & 0.65 & 0.26 & 0.10 & 1.17 \\
\hline & 81 & 0.07 & 22.2 & 11.2 & 2.22 & 0.65 & 0.24 & 0.12 & 1.13 \\
\hline
\end{tabular}




\begin{tabular}{|c|c|c|c|c|c|c|c|c|c|}
\hline & 107 & 0.09 & 21.9 & 10.3 & 1.56 & 0.61 & 0.26 & 0.13 & 1.05 \\
\hline & 120 & 0.10 & 21.8 & 9.86 & 1.16 & 0.58 & 0.26 & 0.15 & 1.15 \\
\hline & 155 & 0.13 & 20.7 & 8.06 & 1.30 & 0.59 & 0.26 & 0.15 & 1.19 \\
\hline & 202 & 0.17 & 20.4 & 9.59 & 2.07 & 0.52 & 0.29 & 0.18 & 1.13 \\
\hline & 224 & 0.19 & 20.1 & 8.98 & 1.57 & 0.49 & 0.30 & 0.20 & 1.08 \\
\hline \multirow[t]{10}{*}{0.76} & 0 & 0.00 & 24.2 & 17.3 & & 0.78 & 0.22 & & 1.12 \\
\hline & 25 & 0.02 & 24.0 & 16.5 & 4.57 & 0.72 & 0.24 & 0.04 & 1.02 \\
\hline & 50 & 0.03 & 22.9 & 11.2 & 0.73 & 0.74 & 0.17 & 0.10 & 1.09 \\
\hline & 80 & 0.05 & 22.5 & 9.70 & 1.36 & 0.75 & 0.18 & 0.07 & 1.04 \\
\hline & 103 & 0.07 & 22.3 & 10.7 & 1.64 & 0.67 & 0.21 & 0.12 & 1.06 \\
\hline & 138 & 0.09 & 21.9 & 9.65 & 1.33 & 0.65 & 0.24 & 0.11 & 1.18 \\
\hline & 150 & 0.10 & 21.4 & 8.40 & 1.03 & 0.63 & 0.24 & 0.13 & 1.15 \\
\hline & 196 & 0.13 & 21.1 & 9.14 & 1.42 & 0.58 & 0.26 & 0.16 & 1.15 \\
\hline & 254 & 0.17 & 20.4 & 9.19 & 1.57 & 0.51 & 0.30 & 0.19 & 1.12 \\
\hline & 281 & 0.19 & 19.8 & 8.51 & 1.57 & 0.51 & 0.29 & 0.20 & 1.29 \\
\hline \multirow[t]{9}{*}{0.91} & 0 & 0.00 & 23.83 & 16.62 & & 0.86 & 0.14 & & 1.11 \\
\hline & 30 & 0.02 & 23.34 & 12.77 & 2.25 & 0.81 & 0.15 & 0.04 & 1.27 \\
\hline & 60 & 0.03 & 23.08 & 12.16 & 2.09 & 0.74 & 0.20 & 0.06 & 1.06 \\
\hline & 92 & 0.05 & 22.33 & 9.29 & 0.50 & 0.64 & 0.19 & 0.18 & 1.19 \\
\hline & 120 & 0.07 & 22.25 & 11.02 & 2.20 & 0.63 & 0.24 & 0.12 & 1.14 \\
\hline & 161 & 0.09 & 21.75 & 10.26 & 2.03 & 0.60 & 0.26 & 0.14 & 1.11 \\
\hline & 232 & 0.13 & 20.09 & 8.86 & 1.54 & 0.49 & 0.30 & 0.20 & 1.10 \\
\hline & 302 & 0.17 & 19.93 & 9.15 & 1.70 & 0.47 & 0.32 & 0.21 & 1.24 \\
\hline & 336 & 0.18 & 19.41 & 8.57 & 1.61 & 0.46 & 0.33 & 0.20 & 1.06 \\
\hline \multirow[t]{6}{*}{1.06} & 0 & 0.00 & 23.6 & 12.3 & & 0.92 & 0.08 & & 1.09 \\
\hline & 37 & 0.02 & 23.9 & 15.1 & 2.53 & 0.74 & 0.22 & 0.04 & 1.10 \\
\hline & 71 & 0.03 & 23.7 & 14.2 & 2.12 & 0.67 & 0.26 & 0.07 & 1.09 \\
\hline & 108 & 0.05 & 22.9 & 12.0 & 1.89 & 0.69 & 0.21 & 0.10 & 1.10 \\
\hline & 140 & 0.07 & 22.6 & 12.3 & 2.86 & 0.65 & 0.23 & 0.12 & 1.08 \\
\hline & 188 & 0.09 & 22.0 & 11.1 & 2.46 & 0.60 & 0.26 & 0.13 & 1.15 \\
\hline
\end{tabular}




\begin{tabular}{|c|c|c|c|c|c|c|c|c|c|}
\hline & 189 & 0.09 & 21.6 & 10.6 & 2.13 & 0.56 & 0.28 & 0.16 & 1.08 \\
\hline & 278 & 0.13 & 20.8 & 10.58 & 2.41 & 0.48 & 0.32 & 0.20 & 1.14 \\
\hline & 386 & 0.18 & 19.0 & 8.70 & 1.75 & 0.43 & 0.33 & 0.23 & 1.17 \\
\hline \multirow[t]{10}{*}{1.21} & 0 & 0.00 & 24.0 & 16.8 & & 0.84 & 0.16 & & 1.19 \\
\hline & 41 & 0.02 & 23.6 & 14.7 & 3.47 & 0.79 & 0.17 & 0.04 & 1.13 \\
\hline & 81 & 0.03 & 23.1 & 11.4 & 1.23 & 0.74 & 0.19 & 0.07 & 1.15 \\
\hline & 128 & 0.05 & 22.5 & 9.63 & 0.65 & 0.69 & 0.19 & 0.12 & 1.17 \\
\hline & 160 & 0.07 & 22.6 & 10.9 & 1.88 & 0.64 & 0.26 & 0.10 & 1.12 \\
\hline & 215 & 0.09 & 21.7 & 9.66 & 1.49 & 0.60 & 0.24 & 0.16 & 1.23 \\
\hline & 237 & 0.10 & 22.1 & 11.4 & 2.02 & 0.54 & 0.29 & 0.17 & 1.21 \\
\hline & 309 & 0.13 & 20.6 & 8.87 & 1.53 & 0.52 & 0.31 & 0.17 & 1.02 \\
\hline & 398 & 0.16 & 19.5 & 9.10 & 1.79 & 0.45 & 0.33 & 0.22 & 1.09 \\
\hline & 446 & 0.18 & 19.0 & 8.51 & 1.68 & 0.44 & 0.34 & 0.22 & 1.12 \\
\hline \multirow[t]{9}{*}{1.36} & 0 & 0.00 & 23.43 & 12.49 & & 0.92 & 0.08 & & 1.06 \\
\hline & 43 & 0.02 & 23.92 & 18.59 & 4.87 & 0.66 & 0.28 & 0.06 & 1.17 \\
\hline & 89 & 0.03 & 22.82 & 10.81 & 1.73 & 0.79 & 0.15 & 0.06 & 1.07 \\
\hline & 140 & 0.05 & 22.51 & 9.85 & 0.83 & 0.69 & 0.18 & 0.13 & 1.04 \\
\hline & 182 & 0.07 & 22.39 & 10.77 & 1.90 & 0.67 & 0.24 & 0.10 & 1.09 \\
\hline & 241 & 0.09 & 21.54 & 8.52 & 0.97 & 0.64 & 0.23 & 0.13 & 1.17 \\
\hline & 270 & 0.10 & 21.18 & 9.47 & 1.45 & 0.56 & 0.27 & 0.17 & 1.10 \\
\hline & 359 & 0.13 & 20.08 & 8.30 & 1.06 & 0.50 & 0.29 & 0.21 & 1.02 \\
\hline & 488 & 0.18 & 19.00 & 8.25 & 1.73 & 0.47 & 0.33 & 0.20 & 1.17 \\
\hline
\end{tabular}

Table 1g: Decay times and pre-exponential factors retrieved by using Equation S1 to fit the fluorescence decays of DNA-EB quenched by Cu ${ }^{2+}$ cations with an $\mathrm{Na}_{2} \mathrm{SO}_{4}$ concentration of $4 \times 10^{-2} \mathrm{M}$.

\begin{tabular}{|c|c|c|c|c|c|c|c|c|c|}
\hline $\begin{array}{c}{[\mathrm{DNA}]} \\
\mathrm{mM} \mathrm{of} \mathrm{bp}\end{array}$ & {$\left[\mathrm{Cu}^{2+}\right](\mu \mathrm{M})$} & {$\left[\mathrm{Cu}^{2+}\right] /[\mathrm{P}]$} & $\begin{array}{c}\tau_{1} \\
\mathrm{~ns}\end{array}$ & $\begin{array}{c}\tau_{2} \\
\mathrm{~ns}\end{array}$ & $\begin{array}{c}\tau_{3} \\
\mathrm{~ns}\end{array}$ & $a_{1}$ & $a_{2}$ \\
\hline 0.30 & 0 & 0.00 & 23.7 & 16.7 & & 0.84 & 0.16 & \\
\hline
\end{tabular}




\begin{tabular}{|c|c|c|c|c|c|c|c|c|c|}
\hline & 9 & 0.01 & 23.6 & 14.5 & & 0.88 & 0.12 & & 1.04 \\
\hline & 20 & 0.03 & 23.7 & 16.9 & & 0.82 & 0.18 & & 1.00 \\
\hline & 40 & 0.07 & 23.0 & 10.0 & & 0.92 & 0.08 & & 1.09 \\
\hline & 59 & 0.10 & 23.0 & 15.1 & 4.21 & 0.61 & 0.27 & 0.12 & 1.10 \\
\hline & 80 & 0.13 & 22.0 & 10.8 & 1.94 & 0.67 & 0.22 & 0.11 & 1.11 \\
\hline & 100 & 0.17 & 21.8 & 11.0 & 2.35 & 0.64 & 0.23 & 0.12 & 1.00 \\
\hline & 116 & 0.19 & 21.5 & 9.98 & 2.08 & 0.64 & 0.25 & 0.11 & 1.01 \\
\hline \multirow[t]{8}{*}{0.45} & 0 & 0.00 & 23.8 & 16.3 & & 0.84 & 0.16 & & 1.08 \\
\hline & 15 & 0.02 & 23.9 & 17.2 & & 0.80 & 0.20 & & 1.08 \\
\hline & 30 & 0.03 & 22.8 & 9.24 & & 0.87 & 0.13 & & 1.07 \\
\hline & 60 & 0.07 & 22.4 & 10.0 & 1.25 & 0.75 & 0.16 & 0.09 & 1.08 \\
\hline & 90 & 0.10 & 22.9 & 13.5 & 2.62 & 0.61 & 0.28 & 0.12 & 1.08 \\
\hline & 120 & 0.13 & 21.9 & 9.72 & 1.43 & 0.64 & 0.23 & 0.12 & 1.13 \\
\hline & 151 & 0.17 & 21.3 & 8.94 & 1.50 & 0.63 & 0.24 & 0.13 & 1.18 \\
\hline & 173 & 0.19 & 21.2 & 9.59 & 1.58 & 0.58 & 0.26 & 0.15 & 1.05 \\
\hline \multirow[t]{9}{*}{0.61} & 0 & 0.00 & 23.8 & 16.2 & & 0.85 & 0.15 & & 0.97 \\
\hline & 10 & 0.01 & 23.8 & 17.1 & & 0.84 & 0.16 & & 1.10 \\
\hline & 20 & 0.02 & 23.9 & 17.3 & & 0.82 & 0.18 & & 1.11 \\
\hline & 39 & 0.03 & 23.0 & 8.86 & & 0.89 & 0.11 & & 1.11 \\
\hline & 80 & 0.07 & 22.5 & 8.49 & & 0.84 & 0.16 & & 1.19 \\
\hline & 119 & 0.10 & 22.3 & 8.09 & & 0.79 & 0.21 & & 1.12 \\
\hline & 160 & 0.13 & 21.8 & 7.35 & & 0.77 & 0.23 & & 1.15 \\
\hline & 201 & 0.17 & 21.7 & 9.39 & 1.85 & 0.65 & 0.23 & 0.11 & 1.09 \\
\hline & 230 & 0.19 & 21.3 & 10.4 & 2.03 & 0.55 & 0.28 & 0.16 & 1.14 \\
\hline \multirow[t]{9}{*}{0.76} & 0 & 0.00 & 23.9 & 18.1 & & 0.81 & 0.19 & & 1.15 \\
\hline & 13 & 0.01 & 23.9 & 16.2 & & 0.84 & 0.16 & & 1.24 \\
\hline & 24 & 0.02 & 23.3 & 10.6 & & 0.89 & 0.11 & & 1.12 \\
\hline & 50 & 0.03 & 22.7 & 8.48 & & 0.87 & 0.13 & & 1.20 \\
\hline & 101 & 0.07 & 22.8 & 8.79 & & 0.85 & 0.15 & & 1.15 \\
\hline & 152 & 0.10 & 22.4 & 11.9 & 2.72 & 0.63 & 0.25 & 0.12 & 1.02 \\
\hline & 203 & 0.13 & 21.2 & 8.83 & 1.36 & 0.58 & 0.26 & 0.16 & 1.03 \\
\hline & 259 & 0.17 & 20.6 & 9.07 & 1.91 & 0.56 & 0.28 & 0.16 & 1.11 \\
\hline & 287 & 0.19 & 20.4 & 9.85 & 2.12 & 0.51 & 0.31 & 0.19 & 1.08 \\
\hline \multirow[t]{2}{*}{0.91} & 0 & 0.00 & 23.8 & 14.6 & & 0.88 & 0.12 & & 1.02 \\
\hline & 15 & 0.01 & 24.1 & 17.5 & & 0.80 & 0.20 & & 1.13 \\
\hline
\end{tabular}




\begin{tabular}{|c|c|c|c|c|c|c|c|c|c|}
\hline & 30 & 0.02 & 23.5 & 12.8 & & 0.86 & 0.14 & & 1.19 \\
\hline & 60 & 0.03 & 23.0 & 9.01 & & 0.86 & 0.14 & & 1.14 \\
\hline & 119 & 0.07 & 22.3 & 7.63 & & 0.81 & 0.19 & & 1.08 \\
\hline & 180 & 0.10 & 21.9 & 9.20 & 1.65 & 0.69 & 0.21 & 0.10 & 1.15 \\
\hline & 231 & 0.13 & 21.3 & 10.5 & 2.44 & 0.58 & 0.25 & 0.17 & 1.14 \\
\hline & 301 & 0.17 & 20.9 & 9.94 & 2.07 & 0.52 & 0.29 & 0.18 & 1.11 \\
\hline & 343 & 0.19 & 20.3 & 9.42 & 2.12 & 0.52 & 0.30 & 0.18 & 1.13 \\
\hline \multirow[t]{9}{*}{1.06} & 0 & 0.00 & 23.9 & 17.2 & & 0.82 & 0.19 & & 1.06 \\
\hline & 18 & 0.01 & 23.7 & 16.9 & & 0.85 & 0.15 & & 1.22 \\
\hline & 35 & 0.02 & 23.3 & 11.8 & & 0.88 & 0.12 & & 1.17 \\
\hline & 71 & 0.03 & 22.7 & 7.95 & & 0.88 & 0.12 & & 1.11 \\
\hline & 140 & 0.07 & 22.6 & 11.4 & 1.45 & 0.73 & 0.19 & 0.08 & 1.15 \\
\hline & 210 & 0.10 & 22.2 & 10.1 & 1.39 & 0.65 & 0.23 & 0.12 & 1.08 \\
\hline & 280 & 0.13 & 20.8 & 9.41 & 1.66 & 0.56 & 0.27 & 0.17 & 1.10 \\
\hline & 357 & 0.17 & 20.1 & 8.30 & 1.55 & 0.55 & 0.28 & 0.16 & 1.05 \\
\hline & 398 & 0.19 & 19.7 & 8.30 & 1.45 & 0.51 & 0.30 & 0.18 & 1.13 \\
\hline \multirow[t]{9}{*}{1.21} & 0 & 0.00 & 24.4 & 18.3 & & 0.85 & 0.15 & & 1.06 \\
\hline & 20 & 0.01 & 24.1 & 17.5 & & 0.20 & 0.80 & & 1.22 \\
\hline & 40 & 0.02 & 23.5 & 12.0 & & 0.14 & 0.86 & & 1.17 \\
\hline & 79 & 0.03 & 24.5 & 18.8 & 5.38 & 0.53 & 0.38 & 0.09 & 1.07 \\
\hline & 160 & 0.07 & 23.0 & 12.3 & 2.31 & 0.66 & 0.24 & 0.10 & 1.07 \\
\hline & 239 & 0.10 & 22.1 & 10.1 & 1.80 & 0.64 & 0.25 & 0.12 & 1.01 \\
\hline & 320 & 0.13 & 20.6 & 8.94 & 1.70 & 0.55 & 0.28 & 0.17 & 1.16 \\
\hline & 404 & 0.17 & 20.0 & 9.10 & 1.97 & 0.50 & 0.32 & 0.19 & 1.01 \\
\hline & 468 & 0.19 & 19.3 & 8.86 & 1.92 & 0.48 & 0.31 & 0.21 & 1.12 \\
\hline \multirow[t]{8}{*}{1.36} & 0 & 0.00 & 24.1 & 12.2 & & 0.93 & 0.07 & & 1.07 \\
\hline & 45 & 0.02 & 23.3 & 11.4 & & 0.90 & 0.10 & & 1.08 \\
\hline & 90 & 0.03 & 23.1 & 9.57 & & 0.90 & 0.10 & & 1.09 \\
\hline & 181 & 0.07 & 23.0 & 13.9 & 3.03 & 0.69 & 0.22 & 0.09 & 1.01 \\
\hline & 279 & 0.10 & 21.7 & 9.63 & 1.41 & 0.64 & 0.23 & 0.14 & 1.24 \\
\hline & 362 & 0.13 & 21.1 & 10.2 & 2.07 & 0.54 & 0.29 & 0.16 & 1.02 \\
\hline & 446 & 0.16 & 20.1 & 8.95 & 1.65 & 0.51 & 0.29 & 0.19 & 1.04 \\
\hline & 515 & 0.19 & 19.6 & 8.73 & 1.65 & 0.49 & 0.31 & 0.20 & 1.10 \\
\hline
\end{tabular}


Table 2a: Decay times and pre-exponential factors retrieved by using Equation $\mathrm{S} 1$ to fit the fluorescence decays of DNA-EB quenched by Ni ${ }^{2+}$ cations with an $\mathrm{Na}_{2} \mathrm{SO}_{4}$ concentration of $5 \times 10^{-4} \mathrm{M}$.

\begin{tabular}{|c|c|c|c|c|c|c|c|c|c|}
\hline $\begin{array}{c}{[\mathrm{DNA}]} \\
\mathrm{mM} \text { of } \mathrm{bp}\end{array}$ & {$\left[\mathrm{Ni}^{2+}\right](\mu \mathrm{M})$} & {$\left[\mathrm{Ni}^{2+}\right] /[\mathrm{P}]$} & $\begin{array}{c}\tau_{1} \\
\mathrm{~ns}\end{array}$ & $\begin{array}{r}\tau_{2} \\
\mathrm{~ns}\end{array}$ & $\begin{array}{r}\tau_{3} \\
\mathrm{~ns}\end{array}$ & $a_{1}$ & $a_{2}$ & $a_{3}$ & $\chi^{2}$ \\
\hline \multirow[t]{10}{*}{0.30} & 0 & 0.00 & 23.2 & 12.1 & & 0.93 & 0.07 & & 1.04 \\
\hline & 12 & 0.02 & 22.7 & 11.5 & 1.20 & 0.79 & 0.13 & 0.09 & 1.09 \\
\hline & 20 & 0.03 & 22.1 & 10.9 & 2.07 & 0.75 & 0.15 & 0.10 & 1.09 \\
\hline & 25 & 0.04 & 22.3 & 13.6 & 2.70 & 0.64 & 0.23 & 0.14 & 1.22 \\
\hline & 34 & 0.06 & 21.4 & 11.1 & 1.89 & 0.57 & 0.24 & 0.19 & 1.10 \\
\hline & 40 & 0.07 & 21.1 & 11.7 & 2.13 & 0.50 & 0.29 & 0.21 & 1.18 \\
\hline & 54 & 0.09 & 19.4 & 10.2 & 1.83 & 0.44 & 0.28 & 0.28 & 1.10 \\
\hline & 60 & 0.10 & 17.9 & 8.66 & 1.70 & 0.44 & 0.27 & 0.30 & 1.15 \\
\hline & 85 & 0.14 & 15.5 & 7.55 & 1.59 & 0.33 & 0.32 & 0.35 & 1.00 \\
\hline & 112 & 0.18 & 13.1 & 6.41 & 1.55 & 0.32 & 0.31 & 0.38 & 1.07 \\
\hline \multirow[t]{11}{*}{0.45} & 0 & 0.00 & 23.2 & 11.7 & & 0.89 & 0.11 & & 1.14 \\
\hline & 19 & 0.02 & 23.0 & 13.7 & 1.62 & 0.76 & 0.17 & 0.07 & 1.05 \\
\hline & 30 & 0.03 & 22.9 & 14.9 & 2.43 & 0.64 & 0.24 & 0.12 & 1.10 \\
\hline & 36 & 0.04 & 22.7 & 14.2 & 2.77 & 0.62 & 0.25 & 0.13 & 1.07 \\
\hline & 50 & 0.05 & 21.2 & 9.89 & 1.62 & 0.63 & 0.20 & 0.17 & 1.07 \\
\hline & 60 & 0.07 & 20.7 & 10.7 & 1.80 & 0.54 & 0.24 & 0.22 & 1.24 \\
\hline & 73 & 0.08 & 19.6 & 9.56 & 1.77 & 0.50 & 0.25 & 0.25 & 1.14 \\
\hline & 80 & 0.09 & 19.4 & 9.66 & 1.82 & 0.47 & 0.26 & 0.26 & 1.08 \\
\hline & 90 & 0.10 & 18.2 & 8.65 & 1.60 & 0.45 & 0.25 & 0.29 & 1.12 \\
\hline & 128 & 0.14 & 16.0 & 8.39 & 1.73 & 0.33 & 0.31 & 0.36 & 1.20 \\
\hline & 168 & 0.18 & 13.0 & 6.46 & 1.63 & 0.33 & 0.28 & 0.39 & 1.18 \\
\hline \multirow[t]{3}{*}{0.76} & 0 & 0.00 & 23.2 & 10.9 & & 0.94 & 0.06 & & 0.99 \\
\hline & 30 & 0.02 & 24.0 & 18.5 & 4.63 & 0.58 & 0.35 & 0.08 & 1.07 \\
\hline & 50 & 0.03 & 23.0 & 14.8 & 2.44 & 0.66 & 0.23 & 0.11 & 1.07 \\
\hline
\end{tabular}




\begin{tabular}{|c|c|c|c|c|c|c|c|c|c|}
\hline & 61 & 0.04 & 22.4 & 13.8 & 2.37 & 0.68 & 0.20 & 0.13 & 1.29 \\
\hline & 83 & 0.05 & 21.5 & 10.7 & 1.95 & 0.64 & 0.20 & 0.17 & 0.98 \\
\hline & 100 & 0.07 & 21.3 & 11.2 & 1.91 & 0.55 & 0.26 & 0.19 & 1.16 \\
\hline & 121 & 0.08 & 20.1 & 9.69 & 1.80 & 0.52 & 0.25 & 0.23 & 1.17 \\
\hline & 150 & 0.10 & 18.3 & 8.17 & 1.69 & 0.49 & 0.23 & 0.28 & 1.10 \\
\hline & 190 & 0.13 & 15.5 & 7.40 & 1.60 & 0.33 & 0.32 & 0.35 & 1.07 \\
\hline & 217 & 0.14 & 14.6 & 7.03 & 1.61 & 0.35 & 0.27 & 0.38 & 1.04 \\
\hline & 283 & 0.19 & 12.6 & 6.92 & 1.70 & 0.27 & 0.29 & 0.43 & 1.15 \\
\hline \multirow[t]{12}{*}{1.06} & 0 & 0.00 & 23.7 & 15.6 & & 0.88 & 0.12 & & 1.16 \\
\hline & 43 & 0.02 & 23.7 & 17.8 & 3.53 & 0.60 & 0.33 & 0.07 & 1.09 \\
\hline & 70 & 0.03 & 22.6 & 13.9 & 2.21 & 0.65 & 0.22 & 0.13 & 1.09 \\
\hline & 85 & 0.04 & 22.3 & 12.4 & 2.08 & 0.58 & 0.26 & 0.16 & 1.05 \\
\hline & 116 & 0.05 & 22.3 & 12.5 & 2.09 & 0.57 & 0.26 & 0.16 & 1.05 \\
\hline & 141 & 0.07 & 21.2 & 10.8 & 1.71 & 0.55 & 0.24 & 0.21 & 1.14 \\
\hline & 169 & 0.08 & 20.1 & 9.40 & 1.66 & 0.53 & 0.24 & 0.24 & 1.23 \\
\hline & 187 & 0.09 & 19.7 & 9.62 & 1.80 & 0.49 & 0.24 & 0.27 & 1.08 \\
\hline & 211 & 0.10 & 19.1 & 9.59 & 1.81 & 0.43 & 0.28 & 0.29 & 1.16 \\
\hline & 270 & 0.13 & 15.5 & 7.50 & 1.58 & 0.34 & 0.32 & 0.34 & 1.07 \\
\hline & 301 & 0.14 & 15.4 & 8.27 & 1.79 & 0.31 & 0.30 & 0.38 & 1.02 \\
\hline & 385 & 0.18 & 12.5 & 6.46 & 1.65 & 0.33 & 0.26 & 0.42 & 1.16 \\
\hline \multirow[t]{9}{*}{1.36} & 0 & 0.00 & 23.5 & 12.2 & & 0.92 & 0.08 & & 1.04 \\
\hline & 59 & 0.02 & 23.9 & 16.4 & 2.45 & 0.64 & 0.29 & 0.07 & 1.12 \\
\hline & 93 & 0.03 & 23.5 & 15.5 & 2.81 & 0.62 & 0.28 & 0.10 & 1.10 \\
\hline & 110 & 0.04 & 23.0 & 13.2 & 1.67 & 0.61 & 0.26 & 0.13 & 0.98 \\
\hline & 153 & 0.06 & 21.6 & 10.7 & 1.71 & 0.61 & 0.22 & 0.18 & 1.04 \\
\hline & 174 & 0.06 & 20.9 & 9.38 & 1.55 & 0.59 & 0.21 & 0.20 & 1.10 \\
\hline & 222 & 0.08 & 20.4 & 10.7 & 1.90 & 0.48 & 0.26 & 0.26 & 1.10 \\
\hline & 244 & 0.09 & 19.6 & 9.69 & 1.96 & 0.48 & 0.26 & 0.26 & 1.07 \\
\hline & 266 & 0.10 & 18.8 & 8.95 & 1.72 & 0.46 & 0.25 & 0.29 & 1.16 \\
\hline
\end{tabular}




\begin{tabular}{|l|l|l|l|l|l|l|l|l|l|l|}
\hline & 350 & 0.13 & 15.5 & 7.50 & 1.59 & 0.33 & 0.32 & 0.34 & 1.08 \\
\cline { 2 - 11 } & 381 & 0.14 & 15.1 & 7.55 & 1.65 & 0.35 & 0.27 & 0.38 & 1.18 \\
\cline { 2 - 11 } & 509 & 0.19 & 12.4 & 6.21 & 1.59 & 0.32 & 0.27 & 0.41 & 1.01 \\
\hline
\end{tabular}

Table 2b: Decay times and pre-exponential factors retrieved by using Equation $\mathrm{S} 1$ to fit the fluorescence decays of DNA-EB quenched by Ni ${ }^{2+}$ cations with an $\mathrm{Na}_{2} \mathrm{SO}_{4}$ concentration of $5 \times 10^{-3} \mathrm{M}$.

\begin{tabular}{|c|c|c|c|c|c|c|c|c|c|}
\hline $\begin{array}{c}{[\mathrm{DNA}]} \\
\mathrm{mM} \text { of } \mathrm{bp}\end{array}$ & {$\left[\mathrm{Ni}^{2+}\right](\mu \mathrm{M})$} & {$\left[\mathrm{Ni}^{2+}\right] /[\mathrm{P}]$} & $\begin{array}{r}\tau_{1} \\
\mathrm{~ns}\end{array}$ & $\begin{array}{r}\tau_{2} \\
\mathrm{~ns}\end{array}$ & $\begin{array}{r}\tau_{3} \\
\mathrm{~ns}\end{array}$ & $a_{1}$ & $a_{2}$ & $a_{3}$ & $\chi^{2}$ \\
\hline \multirow[t]{12}{*}{0.30} & 0 & 0.00 & 23.4 & 13.2 & & 0.90 & 0.10 & & 1.10 \\
\hline & 6 & 0.01 & 23.2 & 13.2 & 2.04 & 0.82 & 0.14 & 0.04 & 1.05 \\
\hline & 12 & 0.02 & 23.1 & 13.9 & 1.66 & 0.74 & 0.19 & 0.07 & 1.06 \\
\hline & 19 & 0.03 & 22.2 & 11.3 & 2.07 & 0.72 & 0.17 & 0.11 & 1.11 \\
\hline & 24 & 0.04 & 22.0 & 10.6 & 1.69 & 0.70 & 0.18 & 0.12 & 1.10 \\
\hline & 30 & 0.05 & 21.6 & 10.8 & 1.82 & 0.64 & 0.20 & 0.16 & 1.09 \\
\hline & 42 & 0.07 & 21.3 & 11.1 & 1.96 & 0.56 & 0.25 & 0.19 & 1.16 \\
\hline & 48 & 0.08 & 20.4 & 9.57 & 1.79 & 0.56 & 0.24 & 0.20 & 1.13 \\
\hline & 55 & 0.09 & 19.5 & 8.92 & 1.57 & 0.52 & 0.24 & 0.24 & 1.18 \\
\hline & 60 & 0.10 & 19.9 & 10.3 & 1.90 & 0.47 & 0.27 & 0.26 & 1.16 \\
\hline & 89 & 0.15 & 17.5 & 8.08 & 1.66 & 0.42 & 0.28 & 0.30 & 1.12 \\
\hline & 118 & 0.20 & 15.5 & 7.58 & 1.63 & 0.42 & 0.38 & 0.20 & 0.96 \\
\hline \multirow[t]{8}{*}{0.45} & 0 & 0.00 & 23.4 & 13.9 & & 0.90 & 0.10 & & 1.07 \\
\hline & 9 & 0.01 & 22.5 & 10.2 & 1.19 & 0.81 & 0.12 & 0.07 & 1.00 \\
\hline & 16 & 0.02 & 22.8 & 11.7 & 1.59 & 0.80 & 0.14 & 0.07 & 1.15 \\
\hline & 30 & 0.03 & 22.4 & 11.7 & 2.00 & 0.72 & 0.18 & 0.10 & 1.21 \\
\hline & 37 & 0.04 & 22.2 & 11.1 & 1.37 & 0.67 & 0.19 & 0.14 & 1.17 \\
\hline & 52 & 0.06 & 22.1 & 11.1 & 1.55 & 0.62 & 0.22 & 0.16 & 1.08 \\
\hline & 60 & 0.07 & 21.0 & 10.6 & 1.73 & 0.58 & 0.23 & 0.19 & 1.21 \\
\hline & 73 & 0.08 & 20.1 & 9.46 & 1.74 & 0.55 & 0.23 & 0.22 & 1.17 \\
\hline
\end{tabular}




\begin{tabular}{|c|c|c|c|c|c|c|c|c|c|}
\hline & 80 & 0.09 & 20.0 & 10.8 & 1.93 & 0.46 & 0.28 & 0.26 & 1.02 \\
\hline & 92 & 0.10 & 19.2 & 9.79 & 1.77 & 0.44 & 0.27 & 0.29 & 1.07 \\
\hline & 129 & 0.14 & 16.9 & 7.76 & 1.74 & 0.43 & 0.26 & 0.31 & 1.17 \\
\hline & 171 & 0.19 & 16.0 & 8.33 & 1.90 & 0.34 & 0.28 & 0.38 & 1.20 \\
\hline \multirow[t]{12}{*}{0.76} & 0 & 0.00 & 25.4 & 19.2 & & 0.56 & 0.44 & & 1.13 \\
\hline & 16 & 0.01 & 23.5 & 13.4 & 0.12 & 0.37 & 0.55 & 0.07 & 1.04 \\
\hline & 30 & 0.02 & 23.1 & 12.7 & 1.42 & 0.77 & 0.16 & 0.07 & 1.09 \\
\hline & 51 & 0.03 & 22.9 & 12.8 & 2.15 & 0.69 & 0.21 & 0.10 & 1.01 \\
\hline & 61 & 0.04 & 22.1 & 10.6 & 1.83 & 0.71 & 0.17 & 0.12 & 1.02 \\
\hline & 77 & 0.05 & 21.8 & 11.2 & 1.87 & 0.64 & 0.21 & 0.16 & 1.06 \\
\hline & 103 & 0.07 & 21.1 & 10.2 & 1.66 & 0.57 & 0.23 & 0.19 & 1.12 \\
\hline & 122 & 0.08 & 20.3 & 8.93 & 1.51 & 0.54 & 0.24 & 0.22 & 1.14 \\
\hline & 131 & 0.09 & 20.1 & 10.0 & 1.96 & 0.51 & 0.24 & 0.25 & 1.09 \\
\hline & 152 & 0.10 & 19.8 & 10.6 & 1.93 & 0.43 & 0.28 & 0.29 & 1.03 \\
\hline & 220 & 0.15 & 16.7 & 8.26 & 1.73 & 0.38 & 0.26 & 0.36 & 1.27 \\
\hline & 280 & 0.19 & 15.9 & 6.86 & 1.10 & 0.24 & 0.26 & 0.50 & 1.20 \\
\hline \multirow[t]{12}{*}{1.06} & 0 & 0.00 & 23.5 & 12.9 & & 0.92 & 0.08 & & 1.03 \\
\hline & 23 & 0.01 & 23.0 & 11.0 & 1.43 & 0.88 & 0.09 & 0.03 & 1.12 \\
\hline & 44 & 0.02 & 22.4 & 10.4 & 1.68 & 0.75 & 0.15 & 0.10 & 1.14 \\
\hline & 71 & 0.03 & 22.7 & 11.7 & 1.80 & 0.73 & 0.16 & 0.11 & 1.09 \\
\hline & 82 & 0.04 & 22.3 & 10.7 & 1.88 & 0.74 & 0.16 & 0.10 & 1.12 \\
\hline & 102 & 0.05 & 22.0 & 10.8 & 1.83 & 0.65 & 0.20 & 0.15 & 1.13 \\
\hline & 141 & 0.07 & 21.9 & 11.2 & 1.87 & 0.58 & 0.24 & 0.18 & 1.22 \\
\hline & 174 & 0.08 & 20.4 & 9.67 & 1.71 & 0.52 & 0.25 & 0.23 & 1.11 \\
\hline & 191 & 0.09 & 19.9 & 9.33 & 1.69 & 0.50 & 0.24 & 0.26 & 1.11 \\
\hline & 210 & 0.10 & 19.8 & 10.3 & 1.90 & 0.45 & 0.27 & 0.28 & 1.04 \\
\hline & 301 & 0.14 & 16.9 & 8.60 & 1.72 & 0.36 & 0.28 & 0.36 & 1.16 \\
\hline & 389 & 0.18 & 14.9 & 7.44 & 1.65 & 0.34 & 0.28 & 0.39 & 1.03 \\
\hline 1.36 & 0 & 0.00 & 23.3 & 11.1 & & 0.94 & 0.06 & & 1.13 \\
\hline
\end{tabular}




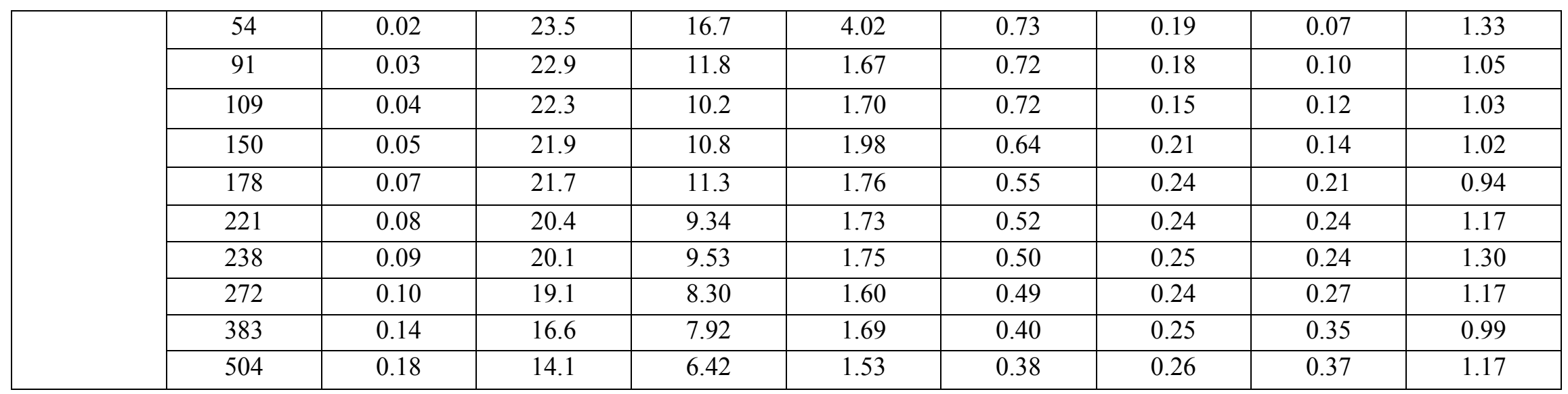

Table 2c: Decay times and pre-exponential factors retrieved by using Equation $\mathrm{S} 1$ to fit the fluorescence decays of DNA-EB quenched by Ni ${ }^{2+}$ cations with an $\mathrm{Na}_{2} \mathrm{SO}_{4}$ concentration of $1.25 \times 10^{-2} \mathrm{M}$.

\begin{tabular}{|c|c|c|c|c|c|c|c|c|c|}
\hline $\begin{array}{c}{[\text { DNA] }} \\
\text { mM of bp }\end{array}$ & {$\left[\mathrm{Ni}^{2+}\right](\mu \mathrm{M})$} & {$\left[\mathrm{Ni}^{2+}\right] /[\mathrm{P}]$} & $\begin{array}{c}\tau_{1} \\
\mathrm{~ns}\end{array}$ & $\begin{array}{c}\tau_{2} \\
\mathrm{~ns}\end{array}$ & $\begin{array}{l}\tau_{3} \\
\mathrm{~ns}\end{array}$ & $a_{1}$ & $a_{2}$ & $a_{3}$ & $\chi^{2}$ \\
\hline \multirow[t]{10}{*}{0.30} & 0 & 0.00 & 23.5 & 13.5 & & 0.90 & 0.10 & & 0.98 \\
\hline & 13 & 0.02 & 23.3 & 16.4 & 4.58 & 0.76 & 0.19 & 0.05 & 1.11 \\
\hline & 21 & 0.03 & 23.7 & 16.9 & 3.27 & 0.61 & 0.30 & 0.09 & 1.29 \\
\hline & 24 & 0.04 & 23.2 & 15.3 & 2.57 & 0.66 & 0.25 & 0.09 & 1.13 \\
\hline & 33 & 0.05 & 23.1 & 14.3 & 2.26 & 0.63 & 0.27 & 0.12 & 1.23 \\
\hline & 40 & 0.07 & 23.2 & 15.9 & 2.65 & 0.53 & 0.33 & 0.14 & 1.18 \\
\hline & 53 & 0.09 & 21.8 & 11.2 & 1.74 & 0.62 & 0.21 & 0.16 & 1.09 \\
\hline & 61 & 0.10 & 21.8 & 11.8 & 2.02 & 0.59 & 0.23 & 0.18 & 1.15 \\
\hline & 85 & 0.14 & 20.8 & 10.9 & 2.00 & 0.51 & 0.26 & 0.22 & 1.04 \\
\hline & 111 & 0.18 & 19.8 & 9.15 & 1.77 & 0.55 & 0.24 & 0.22 & 1.13 \\
\hline \multirow[t]{2}{*}{0.45} & 0 & 0.00 & 23.6 & 14.6 & & 0.89 & 0.11 & & 1.13 \\
\hline & 18 & 0.02 & 23.3 & 14.3 & 2.55 & 0.80 & 0.15 & 0.05 & 0.98 \\
\hline
\end{tabular}




\begin{tabular}{|c|c|c|c|c|c|c|c|c|c|}
\hline & 30 & 0.03 & 22.9 & 12.5 & 0.25 & 0.78 & 0.15 & 0.07 & 1.19 \\
\hline & 37 & 0.04 & 23.4 & 16.2 & 3.11 & 0.63 & 0.27 & 0.10 & 1.28 \\
\hline & 50 & 0.05 & 22.9 & 15.1 & 2.63 & 0.61 & 0.25 & 0.13 & 1.08 \\
\hline & 60 & 0.07 & 22.6 & 12.9 & 2.29 & 0.64 & 0.23 & 0.14 & 1.11 \\
\hline & 72 & 0.08 & 21.7 & 10.2 & 1.64 & 0.65 & 0.20 & 0.15 & 1.30 \\
\hline & 80 & 0.09 & 21.2 & 9.64 & 1.88 & 0.66 & 0.18 & 0.16 & 1.25 \\
\hline & 91 & 0.10 & 21.6 & 11.2 & 1.77 & 0.57 & 0.25 & 0.18 & 1.28 \\
\hline & 127 & 0.14 & 20.6 & 10.4 & 1.97 & 0.53 & 0.25 & 0.22 & 1.03 \\
\hline & 167 & 0.18 & 19.6 & 9.70 & 1.82 & 0.49 & 0.26 & 0.25 & 1.11 \\
\hline \multirow[t]{11}{*}{0.76} & 0 & 0.00 & 23.9 & 15.9 & & 0.85 & 0.15 & & 1.05 \\
\hline & 30 & 0.02 & 23.6 & 18.4 & 4.42 & 0.70 & 0.23 & 0.07 & 1.16 \\
\hline & 49 & 0.03 & 23.7 & 15.8 & 1.98 & 0.63 & 0.27 & 0.09 & 1.20 \\
\hline & 61 & 0.04 & 23.7 & 17.1 & 3.34 & 0.57 & 0.32 & 0.11 & 1.18 \\
\hline & 84 & 0.06 & 22.2 & 9.83 & 1.64 & 0.71 & 0.17 & 0.11 & 1.06 \\
\hline & 100 & 0.07 & 22.1 & 10.9 & 1.80 & 0.66 & 0.20 & 0.14 & 1.13 \\
\hline & 121 & 0.08 & 21.9 & 11.5 & 1.95 & 0.59 & 0.23 & 0.18 & 1.04 \\
\hline & 134 & 0.09 & 21.5 & 11.1 & 1.86 & 0.58 & 0.21 & 0.20 & 1.06 \\
\hline & 150 & 0.10 & 21.7 & 11.6 & 1.80 & 0.52 & 0.27 & 0.21 & 1.04 \\
\hline & 217 & 0.14 & 19.1 & 9.20 & 1.81 & 0.48 & 0.24 & 0.28 & 1.04 \\
\hline & 284 & 0.19 & 18.0 & 8.93 & 1.70 & 0.42 & 0.27 & 0.31 & 1.04 \\
\hline \multirow[t]{9}{*}{1.06} & 0 & 0.00 & 24.5 & 17.9 & & 0.75 & 0.25 & & 1.15 \\
\hline & 42 & 0.02 & 23.8 & 17.3 & 3.84 & 0.71 & 0.23 & 0.06 & 1.07 \\
\hline & 70 & 0.03 & 22.7 & 10.9 & 1.77 & 0.80 & 0.12 & 0.08 & 1.22 \\
\hline & 85 & 0.04 & 23.6 & 16.9 & 3.72 & 0.61 & 0.29 & 0.11 & 1.16 \\
\hline & 117 & 0.05 & 23.9 & 17.1 & 3.00 & 0.46 & 0.40 & 0.14 & 1.15 \\
\hline & 139 & 0.07 & 21.9 & 9.03 & 1.50 & 0.68 & 0.18 & 0.14 & 1.21 \\
\hline & 170 & 0.08 & 21.5 & 10.1 & 1.85 & 0.63 & 0.20 & 0.17 & 1.07 \\
\hline & 186 & 0.09 & 21.7 & 11.0 & 1.64 & 0.56 & 0.24 & 0.20 & 1.19 \\
\hline & 210 & 0.10 & 21.0 & 9.73 & 1.82 & 0.58 & 0.23 & 0.20 & 1.18 \\
\hline
\end{tabular}




\begin{tabular}{|c|c|c|c|c|c|c|c|c|c|}
\hline & 300 & 0.14 & 18.4 & 7.80 & 1.46 & 0.48 & 0.24 & 0.27 & 1.08 \\
\hline & 384 & 0.18 & 17.7 & 8.77 & 1.77 & 0.41 & 0.27 & 0.32 & 1.17 \\
\hline \multirow[t]{10}{*}{1.36} & 0 & 0.00 & 23.7 & 15.1 & & 0.87 & 0.13 & & 1.00 \\
\hline & 57 & 0.02 & 23.4 & 14.9 & 1.98 & 0.74 & 0.20 & 0.06 & 1.15 \\
\hline & 94 & 0.03 & 23.2 & 15.4 & 2.46 & 0.64 & 0.24 & 0.12 & 1.10 \\
\hline & 111 & 0.04 & 22.8 & 14.5 & 2.27 & 0.64 & 0.23 & 0.14 & 1.13 \\
\hline & 156 & 0.06 & 21.8 & 10.1 & 1.47 & 0.65 & 0.19 & 0.16 & 1.09 \\
\hline & 219 & 0.08 & 20.9 & 10.3 & 1.96 & 0.58 & 0.22 & 0.20 & 0.96 \\
\hline & 242 & 0.09 & 20.9 & 10.7 & 1.92 & 0.52 & 0.26 & 0.22 & 1.11 \\
\hline & 272 & 0.10 & 20.3 & 10.1 & 1.72 & 0.50 & 0.26 & 0.24 & 1.00 \\
\hline & 382 & 0.14 & 19.0 & 9.66 & 1.73 & 0.42 & 0.28 & 0.30 & 1.11 \\
\hline & 504 & 0.18 & 17.2 & 8.61 & 1.75 & 0.39 & 0.27 & 0.33 & 1.26 \\
\hline
\end{tabular}

Table 2d: Decay times and pre-exponential factors retrieved by using Equation $\mathrm{S} 1$ to fit the fluorescence decays of DNA-EB quenched by Ni ${ }^{2+}$ cations with an $\mathrm{Na}_{2} \mathrm{SO}_{4}$ concentration of $2.1 \times 10^{-2} \mathrm{M}$.

\begin{tabular}{|c|c|c|c|c|c|c|c|c|c|}
\hline $\begin{array}{l}{[\mathrm{DNA}]} \\
\mathrm{mM} \text { of } \mathrm{bp}\end{array}$ & {$\left[\mathrm{Ni}^{2+}\right](\mu \mathrm{M})$} & {$\left[\mathrm{Ni}^{2+}\right] /[\mathrm{P}]$} & $\begin{array}{r}\tau_{1} \\
\mathrm{~ns}\end{array}$ & $\begin{array}{l}\tau_{2} \\
\mathrm{~ns}\end{array}$ & $\begin{array}{c}\tau_{3} \\
\mathrm{~ns}\end{array}$ & $a_{1}$ & $a_{2}$ & $a_{3}$ & $\chi^{2}$ \\
\hline \multirow[t]{7}{*}{0.30} & 0 & 0.00 & 23.4 & 13.2 & & 0.90 & 0.10 & & 1.07 \\
\hline & 12 & 0.02 & 23.5 & 20.2 & 7.09 & 0.66 & 0.27 & 0.08 & 1.17 \\
\hline & 20 & 0.03 & 23.5 & 16.8 & 3.25 & 0.68 & 0.25 & 0.07 & 1.09 \\
\hline & 40 & 0.07 & 23.2 & 15.4 & 2.62 & 0.62 & 0.27 & 0.11 & 1.13 \\
\hline & 60 & 0.10 & 21.8 & 9.81 & 1.52 & 0.71 & 0.16 & 0.13 & 1.26 \\
\hline & 85 & 0.14 & 21.5 & 9.93 & 1.88 & 0.67 & 0.19 & 0.14 & 1.11 \\
\hline & 112 & 0.18 & 21.1 & 9.54 & 1.51 & 0.62 & 0.22 & 0.16 & 1.07 \\
\hline \multirow[t]{4}{*}{0.45} & 0 & 0.00 & 23.5 & 13.7 & & 0.91 & 0.09 & & 1.11 \\
\hline & 36 & 0.04 & 23.8 & 17.9 & 4.02 & 0.58 & 0.34 & 0.09 & 1.12 \\
\hline & 50 & 0.06 & 23.8 & 18.8 & 4.21 & 0.47 & 0.41 & 0.13 & 1.21 \\
\hline & 60 & 0.07 & 22.7 & 12.8 & 1.80 & 0.68 & 0.21 & 0.11 & 1.04 \\
\hline
\end{tabular}




\begin{tabular}{|c|c|c|c|c|c|c|c|c|c|}
\hline & 80 & 0.09 & 22.4 & 12.8 & 2.14 & 0.64 & 0.22 & 0.14 & 1.09 \\
\hline & 90 & 0.10 & 22.1 & 11.4 & 1.84 & 0.64 & 0.22 & 0.14 & 1.15 \\
\hline & 135 & 0.15 & 20.9 & 8.77 & 1.36 & 0.61 & 0.20 & 0.18 & 1.26 \\
\hline & 167 & 0.18 & 20.7 & 9.96 & 1.78 & 0.57 & 0.23 & 0.20 & 1.10 \\
\hline \multirow[t]{11}{*}{0.76} & 0 & 0.00 & 24.1 & 16.3 & & 0.83 & 0.17 & & 1.07 \\
\hline & 30 & 0.02 & 23.9 & 17.6 & 4.18 & 0.69 & 0.25 & 0.06 & 1.03 \\
\hline & 50 & 0.03 & 23.0 & 11.6 & 1.61 & 0.78 & 0.14 & 0.08 & 1.05 \\
\hline & 61 & 0.04 & 23.0 & 12.7 & 1.56 & 0.72 & 0.17 & 0.11 & 1.05 \\
\hline & 83 & 0.05 & 23.1 & 14.3 & 2.19 & 0.65 & 0.23 & 0.12 & 1.13 \\
\hline & 100 & 0.07 & 22.5 & 11.3 & 1.68 & 0.68 & 0.19 & 0.13 & 1.10 \\
\hline & 121 & 0.08 & 22.0 & 11.7 & 2.07 & 0.66 & 0.18 & 0.16 & 1.20 \\
\hline & 134 & 0.09 & 22.2 & 11.5 & 2.02 & 0.62 & 0.22 & 0.16 & 1.06 \\
\hline & 150 & 0.10 & 21.7 & 10.2 & 1.92 & 0.63 & 0.20 & 0.16 & 1.21 \\
\hline & 215 & 0.14 & 21.0 & 11.0 & 1.88 & 0.50 & 0.27 & 0.23 & 1.06 \\
\hline & 275 & 0.18 & 19.7 & 8.89 & 1.70 & 0.52 & 0.23 & 0.24 & 1.21 \\
\hline \multirow[t]{7}{*}{1.06} & 0 & 0.00 & 23.9 & 13.7 & & 0.88 & 0.12 & & 1.04 \\
\hline & 35 & 0.02 & 23.6 & 14.1 & 2.26 & 0.81 & 0.16 & 0.03 & 1.08 \\
\hline & 70 & 0.03 & 24.1 & 16.3 & 1.78 & 0.61 & 0.30 & 0.09 & 1.07 \\
\hline & 140 & 0.07 & 22.8 & 14.1 & 2.48 & 0.62 & 0.24 & 0.14 & 1.30 \\
\hline & 211 & 0.10 & 21.2 & 9.27 & 1.68 & 0.61 & 0.22 & 0.17 & 1.08 \\
\hline & 348 & 0.16 & 19.8 & 9.33 & 1.54 & 0.49 & 0.26 & 0.25 & 1.08 \\
\hline & 399 & 0.19 & 19.2 & 9.26 & 1.68 & 0.48 & 0.24 & 0.28 & 1.24 \\
\hline \multirow[t]{8}{*}{1.36} & 0 & 0.00 & 23.5 & 11.9 & & 0.93 & 0.07 & & 1.21 \\
\hline & 57 & 0.02 & 23.7 & 14.5 & 1.72 & 0.73 & 0.21 & 0.07 & 1.04 \\
\hline & 99 & 0.04 & 23.0 & 13.0 & 1.64 & 0.71 & 0.19 & 0.10 & 1.14 \\
\hline & 113 & 0.04 & 23.4 & 16.0 & 3.16 & 0.61 & 0.29 & 0.10 & 1.25 \\
\hline & 152 & 0.06 & 22.3 & 10.9 & 1.72 & 0.68 & 0.19 & 0.14 & 1.19 \\
\hline & 246 & 0.09 & 21.1 & 9.12 & 1.60 & 0.62 & 0.20 & 0.18 & 1.13 \\
\hline & 272 & 0.10 & 20.9 & 8.85 & 1.48 & 0.60 & 0.20 & 0.20 & 1.19 \\
\hline & 378 & 0.14 & 19.7 & 8.72 & 1.75 & 0.55 & 0.23 & 0.23 & 1.13 \\
\hline
\end{tabular}




\begin{tabular}{|l|l|l|l|l|l|l|l|l|l|}
\hline & 506 & 0.19 & 18.8 & 8.73 & 1.74 & 0.48 & 0.25 & 0.28 & 1.24 \\
\hline
\end{tabular}

Table 2e: Decay times and pre-exponential factors retrieved by using Equation $\mathrm{S} 1$ to fit the fluorescence decays of DNA-EB quenched by $\mathrm{Ni}^{2+}$ cations with an $\mathrm{Na}_{2} \mathrm{SO}_{4}$ concentration of $3 \times 10^{-2} \mathrm{M}$.

\begin{tabular}{|c|c|c|c|c|c|c|c|c|c|}
\hline $\begin{array}{c}\text { [DNA] } \\
\mathrm{mM} \text { of bp }\end{array}$ & {$\left[\mathrm{Ni}^{2+}\right](\mu \mathrm{M})$} & {$\left[\mathrm{Ni}^{2+}\right] /[\mathrm{P}]$} & $\begin{array}{r}\tau_{1} \\
\mathrm{~ns} \\
\end{array}$ & $\begin{array}{r}\tau_{2} \\
\mathrm{~ns}\end{array}$ & $\begin{array}{r}\tau_{3} \\
\mathrm{~ns}\end{array}$ & $a_{1}$ & $a_{2}$ & $a_{3}$ & $\chi^{2}$ \\
\hline \multirow[t]{8}{*}{0.30} & 0 & 0.00 & 23.00 & 9.35 & & 0.95 & 0.05 & & 1.07 \\
\hline & 13 & 0.02 & 22.90 & 10.90 & & 0.90 & 0.10 & & 1.19 \\
\hline & 20 & 0.03 & 23.10 & 22.70 & 11.5 & 0.47 & 0.38 & 0.15 & 1.13 \\
\hline & 24 & 0.04 & 23.80 & 19.60 & 6.40 & 0.53 & 0.39 & 0.08 & 1.18 \\
\hline & 41 & 0.07 & 23.90 & 16.90 & 2.74 & 0.56 & 0.35 & 0.09 & 1.08 \\
\hline & 60 & 0.10 & 22.10 & 10.80 & 1.48 & 0.74 & 0.17 & 0.09 & 1.03 \\
\hline & 76 & 0.13 & 22.50 & 15.30 & 3.62 & 0.60 & 0.25 & 0.14 & 1.11 \\
\hline & 112 & 0.18 & 21.50 & 10.50 & 1.68 & 0.66 & 0.19 & 0.15 & 1.07 \\
\hline \multirow[t]{11}{*}{0.45} & 0 & 0.00 & 23.9 & 14.9 & & 0.87 & 0.13 & & 1.12 \\
\hline & 18 & 0.02 & 23.9 & 17.2 & 2.04 & 0.69 & 0.27 & 0.04 & 1.11 \\
\hline & 30 & 0.03 & 23.2 & 13.6 & 2.29 & 0.79 & 0.15 & 0.06 & 0.99 \\
\hline & 37 & 0.04 & 23.0 & 13.4 & 2.57 & 0.78 & 0.15 & 0.07 & 1.11 \\
\hline & 50 & 0.06 & 22.7 & 11.5 & 2.27 & 0.78 & 0.15 & 0.07 & 0.96 \\
\hline & 60 & 0.07 & 23.3 & 15.6 & 3.08 & 0.63 & 0.27 & 0.10 & 1.03 \\
\hline & 73 & 0.08 & 22.1 & 9.80 & 1.41 & 0.74 & 0.15 & 0.10 & 1.08 \\
\hline & 80 & 0.09 & 22.8 & 15.0 & 2.88 & 0.63 & 0.25 & 0.18 & 1.04 \\
\hline & 90 & 0.10 & 22.4 & 13.4 & 2.58 & 0.67 & 0.21 & 0.12 & 1.08 \\
\hline & 127 & 0.14 & 21.7 & 10.2 & 1.82 & 0.68 & 0.19 & 0.13 & 1.26 \\
\hline & 168 & 0.18 & 21.3 & 10.0 & 1.86 & 0.64 & 0.21 & 0.16 & 1.10 \\
\hline \multirow[t]{3}{*}{0.76} & 0 & 0.00 & 23.6 & 13.9 & & 0.90 & 0.10 & & 1.17 \\
\hline & 31 & 0.02 & 23.0 & 9.43 & & 0.89 & 0.11 & & 1.11 \\
\hline & 50 & 0.03 & 24.5 & 19.9 & 4.57 & 0.43 & 0.48 & 0.09 & 1.08 \\
\hline
\end{tabular}




\begin{tabular}{|c|c|c|c|c|c|c|c|c|c|}
\hline & 61 & 0.04 & 22.7 & 10.6 & 1.89 & 0.81 & 0.13 & 0.06 & 1.10 \\
\hline & 83 & 0.06 & 23.4 & 15.3 & 2.82 & 0.63 & 0.27 & 0.10 & 1.11 \\
\hline & 100 & 0.07 & 23.3 & 14.9 & 2.49 & 0.61 & 0.27 & 0.12 & 1.05 \\
\hline & 133 & 0.09 & 22.2 & 12.0 & 2.57 & 0.68 & 0.20 & 0.12 & 1.05 \\
\hline & 217 & 0.14 & 21.4 & 10.3 & 1.80 & 0.61 & 0.20 & 0.18 & 0.92 \\
\hline & 283 & 0.19 & 21.0 & 10.2 & 1.73 & 0.56 & 0.24 & 0.20 & 1.08 \\
\hline \multirow[t]{10}{*}{1.06} & 0 & 0.00 & 23.9 & 16.2 & & 0.82 & 0.18 & & 1.07 \\
\hline & 43 & 0.02 & 22.9 & 8.05 & & 0.89 & 0.11 & & 1.11 \\
\hline & 70 & 0.03 & 23.3 & 13.5 & 1.54 & 0.73 & 0.19 & 0.08 & 1.06 \\
\hline & 85 & 0.04 & 22.6 & 9.57 & 1.01 & 0.78 & 0.13 & 0.09 & 1.10 \\
\hline & 117 & 0.05 & 23.4 & 16.0 & 2.78 & 0.59 & 0.30 & 0.12 & 1.11 \\
\hline & 141 & 0.07 & 24.2 & 17.7 & 3.02 & 0.40 & 0.45 & 0.14 & 1.15 \\
\hline & 169 & 0.08 & 22.1 & 11.0 & 1.69 & 0.65 & 0.21 & 0.14 & 1.13 \\
\hline & 210 & 0.10 & 23.0 & 15.2 & 2.35 & 0.53 & 0.31 & 0.16 & 1.13 \\
\hline & 299 & 0.14 & 21.6 & 11.6 & 2.03 & 0.54 & 0.26 & 0.20 & 1.17 \\
\hline & 390 & 0.18 & 21.4 & 13.3 & 2.69 & 0.45 & 0.30 & 0.24 & 1.24 \\
\hline \multirow[t]{9}{*}{1.36} & 0 & 0.00 & 23.6 & 12.9 & & 0.91 & 0.09 & & 1.04 \\
\hline & 33 & 0.01 & 23.2 & 9.45 & & 0.89 & 0.11 & & 1.00 \\
\hline & 94 & 0.03 & 25.5 & 19.0 & 3.37 & 0.37 & 0.53 & 0.10 & 1.05 \\
\hline & 111 & 0.04 & 23.4 & 14.9 & 2.13 & 0.65 & 0.25 & 0.10 & 1.16 \\
\hline & 184 & 0.07 & 22.8 & 13.9 & 2.56 & 0.61 & 0.25 & 0.14 & 1.09 \\
\hline & 219 & 0.08 & 21.9 & 10.7 & 1.74 & 0.66 & 0.20 & 0.15 & 1.24 \\
\hline & 271 & 0.10 & 21.6 & 10.8 & 1.77 & 0.61 & 0.21 & 0.18 & 1.05 \\
\hline & 379 & 0.14 & 21.4 & 12.1 & 2.09 & 0.51 & 0.26 & 0.23 & 1.13 \\
\hline & 510 & 0.19 & 20.2 & 9.76 & 1.76 & 0.51 & 0.24 & 0.25 & 1.12 \\
\hline
\end{tabular}


Table 3a: Decay times and pre-exponential factors retrieved from by using Equation S1 to fit the fluorescence decays of DNA-EB quenched by $\mathrm{Cu}^{2+}$ cations with an $\mathrm{NaCl}$ concentration of $1 \times 10^{-2} \mathrm{M}$.

\begin{tabular}{|c|c|c|c|c|c|c|c|c|c|}
\hline $\begin{array}{c}\text { [DNA] } \\
\mathrm{mM} \text { of } \mathrm{bp}\end{array}$ & {$\left[\mathrm{Cu}^{2+}\right](\mu \mathrm{M})$} & {$\left[\mathrm{Cu}^{2+}\right] /[\mathrm{P}]$} & $\begin{array}{r}\tau_{1} \\
\mathrm{~ns}\end{array}$ & $\begin{array}{r}\tau_{2} \\
\mathrm{~ns}\end{array}$ & $\begin{array}{r}\tau_{3} \\
\mathrm{~ns}\end{array}$ & $a_{1}$ & $a_{2}$ & $a_{3}$ & $\chi^{2}$ \\
\hline \multirow[t]{13}{*}{0.33} & 0 & 0.00 & 23.3 & 11.9 & & 0.89 & 0.11 & & 1.14 \\
\hline & 11 & 0.02 & 22.8 & 12.5 & 2.20 & 0.79 & 0.16 & 0.05 & 0.95 \\
\hline & 18 & 0.03 & 22.1 & 9.40 & 1.06 & 0.76 & 0.16 & 0.08 & 1.11 \\
\hline & 30 & 0.05 & 21.7 & 9.06 & 1.24 & 0.65 & 0.23 & 0.11 & 0.95 \\
\hline & 42 & 0.07 & 21.6 & 12.6 & 3.41 & 0.51 & 0.30 & 0.18 & 1.13 \\
\hline & 52 & 0.09 & 20.0 & 7.52 & 1.08 & 0.57 & 0.28 & 0.15 & 1.04 \\
\hline & 59 & 0.10 & 19.9 & 8.94 & 1.57 & 0.49 & 0.32 & 0.19 & 1.07 \\
\hline & 67 & 0.11 & 19.2 & 8.32 & 1.62 & 0.46 & 0.33 & 0.21 & 1.13 \\
\hline & 80 & 0.13 & 18.1 & 7.47 & 1.42 & 0.42 & 0.34 & 0.24 & 1.21 \\
\hline & 87 & 0.14 & 17.3 & 7.36 & 1.54 & 0.38 & 0.35 & 0.27 & 1.09 \\
\hline & 97 & 0.16 & 17.9 & 8.33 & 1.85 & 0.35 & 0.37 & 0.27 & 1.10 \\
\hline & 102 & 0.17 & 17.1 & 7.98 & 1.74 & 0.31 & 0.39 & 0.31 & 1.12 \\
\hline & 118 & 0.19 & 16.0 & 6.69 & 1.31 & 0.31 & 0.39 & 0.30 & 1.12 \\
\hline \multirow[t]{13}{*}{0.50} & 0 & 0.00 & 23.7 & 15.4 & & 0.85 & 0.15 & & 1.07 \\
\hline & 18 & 0.02 & 23.6 & 14.1 & 2.67 & 0.71 & 0.24 & 0.05 & 1.04 \\
\hline & 26 & 0.03 & 22.2 & 8.69 & 0.35 & 0.67 & 0.14 & 0.19 & 0.95 \\
\hline & 44 & 0.05 & 21.9 & 9.24 & 1.26 & 0.69 & 0.21 & 0.10 & 0.95 \\
\hline & 64 & 0.07 & 21.1 & 9.50 & 1.74 & 0.59 & 0.26 & 0.15 & 1.07 \\
\hline & 82 & 0.09 & 22.1 & 12.5 & 2.49 & 0.39 & 0.40 & 0.21 & 1.04 \\
\hline & 92 & 0.10 & 19.9 & 8.94 & 1.46 & 0.47 & 0.33 & 0.20 & 1.02 \\
\hline & 108 & 0.12 & 19.0 & 8.34 & 1.74 & 0.46 & 0.32 & 0.22 & 1.13 \\
\hline & 118 & 0.13 & 18.6 & 8.07 & 1.59 & 0.42 & 0.35 & 0.23 & 1.01 \\
\hline & 133 & 0.15 & 18.0 & 7.93 & 1.72 & 0.39 & 0.35 & 0.26 & 1.03 \\
\hline & 149 & 0.16 & 17.3 & 7.98 & 1.80 & 0.31 & 0.39 & 0.30 & 1.20 \\
\hline & 159 & 0.17 & 16.7 & 7.38 & 1.50 & 0.32 & 0.39 & 0.29 & 1.19 \\
\hline & 174 & 0.19 & 14.4 & 6.46 & 1.48 & 0.26 & 0.39 & 0.34 & 1.18 \\
\hline
\end{tabular}




\begin{tabular}{|c|c|c|c|c|c|c|c|c|c|}
\hline \multirow[t]{13}{*}{0.62} & 0 & 0.00 & 23.4 & 11.9 & & 0.92 & 0.08 & & 1.13 \\
\hline & 23 & 0.02 & 22.5 & 9.24 & 0.55 & 0.75 & 0.11 & 0.14 & 1.11 \\
\hline & 35 & 0.03 & 22.5 & 10.4 & 1.29 & 0.73 & 0.19 & 0.08 & 1.05 \\
\hline & 58 & 0.05 & 21.5 & 8.12 & 0.54 & 0.61 & 0.22 & 0.18 & 0.94 \\
\hline & 80 & 0.07 & 21.0 & 9.18 & 1.69 & 0.60 & 0.27 & 0.14 & 1.07 \\
\hline & 101 & 0.09 & 20.5 & 8.97 & 1.48 & 0.51 & 0.31 & 0.18 & 1.01 \\
\hline & 116 & 0.10 & 19.8 & 8.52 & 1.64 & 0.50 & 0.32 & 0.19 & 1.05 \\
\hline & 132 & 0.12 & 18.7 & 7.50 & 1.58 & 0.48 & 0.32 & 0.20 & 1.12 \\
\hline & 151 & 0.13 & 18.1 & 7.95 & 1.66 & 0.39 & 0.36 & 0.25 & 1.09 \\
\hline & 166 & 0.15 & 17.2 & 7.21 & 1.47 & 0.36 & 0.38 & 0.26 & 1.13 \\
\hline & 183 & 0.16 & 16.5 & 7.55 & 1.69 & 0.31 & 0.38 & 0.30 & 1.08 \\
\hline & 195 & 0.17 & 15.9 & 6.92 & 1.48 & 0.33 & 0.38 & 0.30 & 1.04 \\
\hline & 218 & 0.19 & 15.1 & 6.90 & 1.65 & 0.28 & 0.38 & 0.34 & 1.08 \\
\hline \multirow[t]{13}{*}{0.73} & 0 & 0.00 & 25.8 & 19.7 & & 0.45 & 0.55 & & 1.07 \\
\hline & 27 & 0.02 & 22.5 & 9.01 & 0.20 & 0.57 & 0.09 & 0.34 & 1.05 \\
\hline & 42 & 0.03 & 22.7 & 11.5 & 1.62 & 0.73 & 0.19 & 0.08 & 0.95 \\
\hline & 69 & 0.05 & 22.0 & 10.4 & 1.72 & 0.65 & 0.24 & 0.11 & 0.94 \\
\hline & 92 & 0.07 & 21.2 & 9.30 & 1.94 & 0.61 & 0.26 & 0.13 & 1.14 \\
\hline & 124 & 0.09 & 20.6 & 9.61 & 1.84 & 0.50 & 0.31 & 0.19 & 0.93 \\
\hline & 138 & 0.10 & 19.9 & 8.46 & 1.46 & 0.51 & 0.30 & 0.19 & 1.16 \\
\hline & 159 & 0.12 & 19.6 & 8.55 & 1.64 & 0.46 & 0.34 & 0.20 & 1.08 \\
\hline & 176 & 0.13 & 17.7 & 7.66 & 1.36 & 0.37 & 0.37 & 0.26 & 0.89 \\
\hline & 195 & 0.14 & 17.4 & 7.57 & 1.53 & 0.37 & 0.38 & 0.25 & 1.15 \\
\hline & 219 & 0.16 & 17.0 & 7.60 & 1.61 & 0.33 & 0.38 & 0.29 & 1.07 \\
\hline & 230 & 0.17 & 16.8 & 7.46 & 1.52 & 0.33 & 0.39 & 0.29 & 1.13 \\
\hline & 264 & 0.19 & 15.3 & 7.33 & 1.72 & 0.24 & 0.40 & 0.35 & 1.22 \\
\hline \multirow[t]{5}{*}{0.98} & 0 & 0.00 & 23.4 & 11.3 & & 0.92 & 0.07 & & 0.97 \\
\hline & 36 & 0.02 & 22.8 & 10.5 & 1.14 & 0.81 & 0.12 & 0.07 & 1.07 \\
\hline & 61 & 0.03 & 24.6 & 16.7 & 3.88 & 0.48 & 0.42 & 0.10 & 1.03 \\
\hline & 94 & 0.05 & 22.3 & 10.6 & 1.83 & 0.64 & 0.25 & 0.10 & 0.99 \\
\hline & 130 & 0.07 & 22.7 & 12.8 & 2.55 & 0.50 & 0.34 & 0.16 & 0.91 \\
\hline
\end{tabular}




\begin{tabular}{|c|c|c|c|c|c|c|c|c|c|}
\hline & 166 & 0.09 & 21.0 & 10.6 & 2.51 & 0.48 & 0.33 & 0.19 & 1.00 \\
\hline & 181 & 0.10 & 20.9 & 9.97 & 1.93 & 0.46 & 0.35 & 0.19 & 1.09 \\
\hline & 237 & 0.13 & 18.8 & 9.12 & 1.99 & 0.36 & 0.37 & 0.27 & 1.10 \\
\hline & 310 & 0.17 & 15.4 & 6.96 & 1.56 & 0.30 & 0.38 & 0.32 & 1.16 \\
\hline & 344 & 0.19 & 15.1 & 7.02 & 1.69 & 0.26 & 0.42 & 0.33 & 1.01 \\
\hline \multirow[t]{13}{*}{1.11} & 0 & 0.00 & 23.2 & 11.2 & & 0.93 & 0.07 & & 1.27 \\
\hline & 43 & 0.02 & 22.6 & 8.67 & 0.46 & 0.76 & 0.12 & 0.12 & 1.15 \\
\hline & 62 & 0.03 & 22.4 & 9.76 & 1.83 & 0.77 & 0.17 & 0.05 & 1.05 \\
\hline & 103 & 0.05 & 22.0 & 10.7 & 1.88 & 0.64 & 0.25 & 0.12 & 1.10 \\
\hline & 146 & 0.07 & 20.6 & 8.08 & 1.33 & 0.59 & 0.27 & 0.14 & 1.07 \\
\hline & 186 & 0.09 & 20.0 & 8.86 & 1.72 & 0.49 & 0.32 & 0.19 & 0.99 \\
\hline & 204 & 0.10 & 19.7 & 8.52 & 1.66 & 0.49 & 0.31 & 0.20 & 1.06 \\
\hline & 236 & 0.12 & 18.9 & 8.38 & 1.77 & 0.43 & 0.34 & 0.22 & 1.01 \\
\hline & 264 & 0.13 & 17.9 & 7.74 & 1.54 & 0.37 & 0.37 & 0.25 & 1.01 \\
\hline & 293 & 0.14 & 16.6 & 7.50 & 1.73 & 0.34 & 0.37 & 0.29 & 0.97 \\
\hline & 327 & 0.16 & 15.7 & 7.97 & 2.43 & 0.29 & 0.38 & 0.33 & 1.07 \\
\hline & 352 & 0.17 & 14.7 & 6.49 & 1.57 & 0.29 & 0.39 & 0.31 & 1.14 \\
\hline & 391 & 0.19 & 13.8 & 6.32 & 1.49 & 0.25 & 0.34 & 0.41 & 1.05 \\
\hline \multirow[t]{13}{*}{1.23} & 0 & 0.00 & 23.1 & 10.3 & & 0.95 & 0.05 & & 1.06 \\
\hline & 46 & 0.02 & 22.8 & 10.5 & 1.08 & 0.81 & 0.14 & 0.05 & 1.01 \\
\hline & 69 & 0.03 & 22.7 & 11.1 & 1.03 & 0.73 & 0.18 & 0.10 & 1.08 \\
\hline & 114 & 0.05 & 22.2 & 10.9 & 1.49 & 0.65 & 0.23 & 0.12 & 1.07 \\
\hline & 162 & 0.07 & 21.2 & 9.11 & 1.48 & 0.60 & 0.26 & 0.13 & 1.09 \\
\hline & 204 & 0.09 & 20.6 & 9.53 & 2.11 & 0.52 & 0.31 & 0.17 & 0.99 \\
\hline & 227 & 0.10 & 19.8 & 9.32 & 2.05 & 0.48 & 0.32 & 0.20 & 0.96 \\
\hline & 263 & 0.12 & 18.7 & 8.20 & 1.69 & 0.43 & 0.33 & 0.24 & 0.99 \\
\hline & 298 & 0.13 & 18.7 & 8.70 & 1.82 & 0.38 & 0.37 & 0.25 & 1.00 \\
\hline & 325 & 0.14 & 17.3 & 7.62 & 1.70 & 0.37 & 0.36 & 0.27 & 1.07 \\
\hline & 363 & 0.16 & 16.5 & 7.49 & 1.67 & 0.33 & 0.39 & 0.28 & 1.10 \\
\hline & 387 & 0.17 & 14.9 & 6.78 & 1.63 & 0.30 & 0.38 & 0.33 & 1.09 \\
\hline & 435 & 0.19 & 13.9 & 6.17 & 1.44 & 0.27 & 0.39 & 0.34 & 1.05 \\
\hline
\end{tabular}


Table 3b: Decay times and pre-exponential factors retrieved from by using Equation S1 to fit the fluorescence decays of DNA-EB quenched by $\mathrm{Cu}^{2+}$ cations with an $\mathrm{NaCl}$ concentration of $2.5 \times 10^{-2} \mathrm{M}$.

\begin{tabular}{|c|c|c|c|c|c|c|c|c|c|}
\hline wt \% DNA & {$\left[\mathrm{Cu}^{2+}\right](\mu \mathrm{M})$} & {$\left[\mathrm{Cu}^{2+}\right] /[\mathrm{P}]$} & $\begin{array}{c}\tau_{1} \\
\mathrm{~ns}\end{array}$ & $\begin{array}{r}\tau_{2} \\
\mathrm{~ns}\end{array}$ & $\begin{array}{r}\tau_{3} \\
\mathrm{~ns}\end{array}$ & $a_{1}$ & $a_{2}$ & $a_{3}$ & $\chi^{2}$ \\
\hline \multirow[t]{13}{*}{0.30} & 0 & 0.00 & 23.1 & 11.3 & & 0.91 & 0.09 & & 1.04 \\
\hline & 12 & 0.02 & 22.4 & 8.89 & 1.39 & 0.85 & 0.11 & 0.04 & 1.12 \\
\hline & 17 & 0.03 & 22.3 & 9.84 & 1.67 & 0.78 & 0.17 & 0.06 & 1.08 \\
\hline & 28 & 0.05 & 22.9 & 14.0 & 3.59 & 0.59 & 0.30 & 0.12 & 0.95 \\
\hline & 42 & 0.07 & 21.7 & 10.7 & 2.39 & 0.63 & 0.25 & 0.12 & 1.09 \\
\hline & 54 & 0.09 & 21.0 & 9.54 & 1.89 & 0.58 & 0.27 & 0.15 & 1.05 \\
\hline & 61 & 0.10 & 20.7 & 9.80 & 2.18 & 0.54 & 0.28 & 0.17 & 1.02 \\
\hline & 69 & 0.11 & 20.3 & 9.39 & 1.94 & 0.49 & 0.31 & 0.19 & 1.07 \\
\hline & 79 & 0.13 & 20.4 & 9.79 & 1.93 & 0.42 & 0.36 & 0.22 & 1.08 \\
\hline & 86 & 0.14 & 19.3 & 8.53 & 1.81 & 0.48 & 0.31 & 0.21 & 1.00 \\
\hline & 96 & 0.16 & 18.7 & 7.78 & 1.51 & 0.45 & 0.34 & 0.21 & 1.11 \\
\hline & 101 & 0.17 & 18.8 & 8.36 & 1.65 & 0.42 & 0.35 & 0.23 & 1.05 \\
\hline & 123 & 0.20 & 18.1 & 8.71 & 2.11 & 0.36 & 0.36 & 0.28 & 1.12 \\
\hline \multirow[t]{11}{*}{0.45} & 0 & 0.00 & 23.3 & 11.3 & & 0.93 & 0.07 & & 1.06 \\
\hline & 17 & 0.02 & 23.6 & 14.4 & 1.72 & 0.74 & 0.21 & 0.05 & 1.13 \\
\hline & 27 & 0.03 & 22.6 & 9.95 & 1.29 & 0.77 & 0.17 & 0.07 & 1.14 \\
\hline & 44 & 0.05 & 22.7 & 12.3 & 2.36 & 0.66 & 0.24 & 0.10 & 0.93 \\
\hline & 63 & 0.07 & 21.9 & 10.6 & 2.22 & 0.62 & 0.24 & 0.13 & 1.08 \\
\hline & 81 & 0.09 & 21.2 & 9.81 & 1.92 & 0.58 & 0.27 & 0.15 & 0.93 \\
\hline & 89 & 0.10 & 21.2 & 10.1 & 1.69 & 0.52 & 0.30 & 0.18 & 1.03 \\
\hline & 104 & 0.11 & 20.4 & 9.31 & 1.98 & 0.51 & 0.30 & 0.19 & 1.05 \\
\hline & 133 & 0.15 & 20.0 & 9.04 & 2.08 & 0.49 & 0.32 & 0.19 & 0.91 \\
\hline & 153 & 0.17 & 18.5 & 8.06 & 1.73 & 0.42 & 0.33 & 0.25 & 1.14 \\
\hline & 176 & 0.19 & 17.6 & 7.50 & 1.53 & 0.37 & 0.36 & 0.27 & 1.09 \\
\hline
\end{tabular}




\begin{tabular}{|c|c|c|c|c|c|c|c|c|c|}
\hline \multirow[t]{12}{*}{0.61} & 0 & 0.00 & 24.3 & 16.7 & & 0.77 & 0.23 & & 1.07 \\
\hline & 23 & 0.02 & 23.0 & 10.1 & 0.56 & 0.83 & 0.11 & 0.06 & 1.02 \\
\hline & 35 & 0.03 & 23.0 & 10.4 & 1.15 & 0.80 & 0.14 & 0.06 & 0.97 \\
\hline & 84 & 0.07 & 22.1 & 10.1 & 1.69 & 0.67 & 0.21 & 0.12 & 1.01 \\
\hline & 112 & 0.09 & 21.6 & 9.05 & 1.20 & 0.62 & 0.24 & 0.14 & 1.11 \\
\hline & 123 & 0.10 & 21.7 & 10.2 & 1.85 & 0.59 & 0.27 & 0.14 & 0.95 \\
\hline & 145 & 0.12 & 21.1 & 9.62 & 2.16 & 0.58 & 0.28 & 0.28 & 1.12 \\
\hline & 162 & 0.13 & 20.4 & 9.29 & 1.83 & 0.50 & 0.31 & 0.20 & 0.97 \\
\hline & 177 & 0.15 & 18.5 & 7.52 & 1.41 & 0.44 & 0.34 & 0.22 & 1.09 \\
\hline & 195 & 0.16 & 19.7 & 9.97 & 2.62 & 0.42 & 0.33 & 0.25 & 1.14 \\
\hline & 206 & 0.17 & 19.7 & 9.36 & 1.98 & 0.41 & 0.35 & 0.25 & 0.99 \\
\hline & 229 & 0.19 & 18.6 & 8.49 & 1.97 & 0.38 & 0.37 & 0.25 & 0.97 \\
\hline \multirow[t]{10}{*}{0.76} & 0 & 0.00 & 23.2 & 11.4 & & 0.93 & 0.07 & & 1.09 \\
\hline & 108 & 0.07 & 21.6 & 9.90 & 1.85 & 0.62 & 0.25 & 0.14 & 1.18 \\
\hline & 137 & 0.09 & 21.3 & 10.5 & 2.07 & 0.55 & 0.29 & 0.17 & 1.13 \\
\hline & 151 & 0.10 & 20.7 & 9.00 & 1.56 & 0.55 & 0.29 & 0.16 & 1.01 \\
\hline & 175 & 0.12 & 20.6 & 9.70 & 1.76 & 0.46 & 0.34 & 0.20 & 1.16 \\
\hline & 198 & 0.13 & 19.6 & 9.16 & 2.00 & 0.44 & 0.34 & 0.22 & 1.11 \\
\hline & 219 & 0.14 & 19.4 & 9.59 & 2.13 & 0.40 & 0.36 & 0.24 & 1.02 \\
\hline & 247 & 0.16 & 17.8 & 8.27 & 1.85 & 0.37 & 0.35 & 0.28 & 1.08 \\
\hline & 254 & 0.17 & 18.4 & 8.96 & 2.01 & 0.33 & 0.39 & 0.28 & 1.19 \\
\hline & 280 & 0.18 & 16.5 & 7.31 & 1.75 & 0.35 & 0.37 & 0.28 & 1.07 \\
\hline \multirow[t]{9}{*}{0.91} & 0 & 0.00 & 23.0 & 7.76 & & 0.96 & 0.04 & & 0.99 \\
\hline & 24 & 0.01 & 23.0 & 10.5 & 0.00 & 0.88 & 0.12 & 0.00 & 0.99 \\
\hline & 53 & 0.03 & 22.7 & 10.1 & 0.94 & 0.78 & 0.14 & 0.08 & 1.17 \\
\hline & 76 & 0.04 & 22.2 & 9.29 & 1.03 & 0.73 & 0.18 & 0.09 & 1.06 \\
\hline & 104 & 0.06 & 21.9 & 9.27 & 1.33 & 0.69 & 0.22 & 0.10 & 1.17 \\
\hline & 130 & 0.07 & 21.7 & 10.5 & 1.86 & 0.59 & 0.27 & 0.14 & 1.05 \\
\hline & 153 & 0.08 & 21.1 & 9.67 & 2.00 & 0.59 & 0.26 & 0.15 & 1.01 \\
\hline & 180 & 0.10 & 20.7 & 9.83 & 2.43 & 0.53 & 0.29 & 0.18 & 1.11 \\
\hline & 204 & 0.11 & 19.6 & 8.69 & 2.01 & 0.52 & 0.30 & 0.18 & 1.09 \\
\hline
\end{tabular}




\begin{tabular}{|c|c|c|c|c|c|c|c|c|c|}
\hline & 233 & 0.13 & 19.1 & 8.27 & 1.58 & 0.45 & 0.33 & 0.22 & 1.11 \\
\hline & 258 & 0.14 & 18.4 & 7.81 & 1.60 & 0.43 & 0.34 & 0.23 & 1.06 \\
\hline & 284 & 0.16 & 18.1 & 8.38 & 1.83 & 0.37 & 0.37 & 0.26 & 1.11 \\
\hline & 308 & 0.17 & 17.0 & 7.59 & 1.57 & 0.34 & 0.38 & 0.28 & 1.20 \\
\hline \multirow[t]{13}{*}{1.06} & 0 & 0.00 & 23.2 & 9.55 & & 0.94 & 0.06 & & 1.10 \\
\hline & 42 & 0.02 & 23.9 & 14.4 & 1.43 & 0.69 & 0.26 & 0.05 & 1.03 \\
\hline & 64 & 0.03 & 22.6 & 10.5 & 1.63 & 0.77 & 0.17 & 0.06 & 1.12 \\
\hline & 109 & 0.05 & 23.0 & 13.1 & 2.31 & 0.60 & 0.28 & 0.11 & 1.06 \\
\hline & 149 & 0.07 & 21.9 & 10.5 & 2.20 & 0.63 & 0.25 & 0.12 & 1.01 \\
\hline & 192 & 0.09 & 20.7 & 8.65 & 1.43 & 0.57 & 0.26 & 0.17 & 1.19 \\
\hline & 214 & 0.10 & 20.5 & 9.32 & 1.79 & 0.54 & 0.29 & 0.17 & 1.16 \\
\hline & 245 & 0.12 & 20.3 & 9.57 & 1.99 & 0.46 & 0.34 & 0.20 & 1.09 \\
\hline & 274 & 0.13 & 19.7 & 8.67 & 1.47 & 0.44 & 0.35 & 0.21 & 1.17 \\
\hline & 305 & 0.14 & 18.9 & 8.98 & 1.98 & 0.40 & 0.35 & 0.25 & 1.00 \\
\hline & 338 & 0.16 & 17.5 & 7.65 & 1.57 & 0.38 & 0.36 & 0.26 & 1.05 \\
\hline & 358 & 0.17 & 17.5 & 8.41 & 1.83 & 0.31 & 0.40 & 0.29 & 1.08 \\
\hline & 400 & 0.19 & 15.6 & 7.22 & 1.64 & 0.30 & 0.38 & 0.32 & 1.05 \\
\hline
\end{tabular}

Table 3c: Decay times and pre-exponential factors retrieved from by using Equation S1 to fit the fluorescence decays of DNA-EB quenched by $\mathrm{Cu}^{2+}$ cations with an $\mathrm{NaCl}$ concentration of $4.1 \times 10^{-2} \mathrm{M}$.

\begin{tabular}{|c|c|c|c|c|c|c|c|c|c|}
\hline $\begin{array}{c}{[\mathrm{DNA}]} \\
\text { mM of bp }\end{array}$ & {$\left[\mathrm{Cu}^{2+}\right](\mu \mathrm{M})$} & {$\left[\mathrm{Cu}^{2+}\right] /[\mathrm{P}]$} & $\begin{array}{c}\tau_{1} \\
\mathrm{~ns}\end{array}$ & $\begin{array}{c}\tau_{2} \\
\mathrm{~ns}\end{array}$ & $\begin{array}{c}\tau_{3} \\
\mathrm{~ns}\end{array}$ & $a_{1}$ & $a_{2}$ & $a_{3}$ & $\chi^{2}$ \\
\hline \multirow{6}{*}{0.30} & 0 & 0.00 & 23.1 & 10.7 & & 0.94 & 0.06 & & 1.08 \\
\cline { 2 - 10 } & 8 & 0.01 & 22.9 & 10.5 & & 0.90 & 0.10 & \\
\cline { 2 - 10 } & 17 & 0.03 & 22.5 & 8.96 & 0.69 & 0.80 & 0.11 & 0.09 & 1.23 \\
\cline { 2 - 11 } & 34 & 0.06 & 22.4 & 10.8 & 1.47 & 0.71 & 0.20 & 0.09 & 1.02 \\
\cline { 2 - 10 } & 41 & 0.07 & 22.0 & 9.99 & 1.75 & 0.71 & 0.21 & 0.08 & 1.01 \\
\cline { 2 - 10 } & 52 & 0.09 & 22.0 & 11.20 & 2.51 & 0.58 & 0.29 & 0.13 & 1.17 \\
\cline { 2 - 10 } & 61 & 0.10 & 21.4 & 9.74 & 1.87 & 0.64 & 0.24 & 0.12 & 1.08 \\
\hline
\end{tabular}




\begin{tabular}{|c|c|c|c|c|c|c|c|c|c|}
\hline & 70 & 0.12 & 21.5 & 10.3 & 1.95 & 0.60 & 0.26 & 0.14 & 1.02 \\
\hline & 76 & 0.13 & 20.9 & 8.83 & 1.45 & 0.59 & 0.26 & 0.15 & 1.03 \\
\hline & 87 & 0.14 & 20.5 & 8.61 & 1.58 & 0.56 & 0.26 & 0.17 & 1.11 \\
\hline & 95 & 0.16 & 20.2 & 9.16 & 1.86 & 0.52 & 0.31 & 0.18 & 0.97 \\
\hline \multirow[t]{11}{*}{0.45} & 0 & 0.00 & 23.2 & 10.4 & & 0.92 & 0.08 & & 0.99 \\
\hline & 18 & 0.02 & 22.8 & 9.81 & 0.12 & 0.38 & 0.06 & 0.56 & 1.10 \\
\hline & 26 & 0.03 & 22.6 & 10.2 & 1.86 & 0.81 & 0.15 & 0.04 & 1.08 \\
\hline & 46 & 0.05 & 22.2 & 10.3 & 1.68 & 0.71 & 0.20 & 0.09 & 1.12 \\
\hline & 63 & 0.07 & 22.6 & 12.0 & 1.70 & 0.58 & 0.30 & 0.12 & 1.11 \\
\hline & 81 & 0.09 & 21.0 & 9.13 & 1.78 & 0.63 & 0.24 & 0.13 & 1.07 \\
\hline & 92 & 0.10 & 21.2 & 10.1 & 1.93 & 0.56 & 0.28 & 0.15 & 1.03 \\
\hline & 104 & 0.11 & 20.4 & 9.15 & 1.81 & 0.54 & 0.28 & 0.17 & 1.14 \\
\hline & 134 & 0.15 & 19.6 & 8.69 & 1.62 & 0.49 & 0.31 & 0.21 & 1.07 \\
\hline & 154 & 0.17 & 19.0 & 8.65 & 1.91 & 0.44 & 0.33 & 0.33 & 1.14 \\
\hline & 173 & 0.19 & 18.3 & 8.08 & 1.55 & 0.41 & 0.35 & 0.25 & 1.13 \\
\hline \multirow[t]{13}{*}{0.61} & 0 & 0.00 & 23.3 & 10.4 & & 0.93 & 0.07 & & 1.18 \\
\hline & 17 & 0.01 & 23.1 & 10.2 & & 0.89 & 0.11 & & 1.02 \\
\hline & 33 & 0.03 & 22.9 & 9.22 & & 0.87 & 0.13 & & 1.12 \\
\hline & 53 & 0.04 & 22.7 & 10.3 & 1.45 & 0.76 & 0.18 & 0.05 & 1.12 \\
\hline & 69 & 0.06 & 22.6 & 10.9 & 1.88 & 0.72 & 0.19 & 0.09 & 1.14 \\
\hline & 86 & 0.07 & 21.9 & 9.13 & 1.60 & 0.71 & 0.20 & 0.09 & 1.07 \\
\hline & 103 & 0.08 & 21.9 & 10.5 & 1.95 & 0.65 & 0.24 & 0.12 & 1.05 \\
\hline & 120 & 0.10 & 21.6 & 10.7 & 2.76 & 0.60 & 0.26 & 0.14 & 1.05 \\
\hline & 136 & 0.11 & 21.1 & 8.78 & 1.28 & 0.59 & 0.26 & 0.15 & 1.09 \\
\hline & 155 & 0.13 & 20.8 & 9.62 & 2.07 & 0.54 & 0.29 & 0.17 & 1.11 \\
\hline & 172 & 0.14 & 21.0 & 10.9 & 2.20 & 0.47 & 0.32 & 0.21 & 1.00 \\
\hline & 188 & 0.16 & 20.4 & 10.0 & 2.06 & 0.44 & 0.34 & 0.21 & 1.04 \\
\hline & 204 & 0.17 & 19.3 & 3.59 & 1.67 & 0.45 & 0.34 & 0.21 & 0.97 \\
\hline \multirow[t]{3}{*}{0.76} & 0 & 0.00 & 23.1 & 10.8 & & 0.94 & 0.06 & & 1.03 \\
\hline & 29 & 0.02 & 22.8 & 9.44 & 0.12 & 0.36 & 0.05 & 0.59 & 1.01 \\
\hline & 45 & 0.03 & 22.7 & 9.84 & 1.11 & 0.82 & 0.13 & 0.05 & 1.13 \\
\hline
\end{tabular}




\begin{tabular}{|c|c|c|c|c|c|c|c|c|c|}
\hline & 75 & 0.05 & 22.5 & 10.9 & 1.84 & 0.71 & 0.21 & 0.08 & 1.00 \\
\hline & 106 & 0.07 & 21.9 & 9.79 & 1.67 & 0.70 & 0.20 & 0.09 & 1.04 \\
\hline & 137 & 0.09 & 22.3 & 12.0 & 2.37 & 0.57 & 0.29 & 0.14 & 1.03 \\
\hline & 154 & 0.10 & 21.1 & 9.62 & 1.61 & 0.56 & 0.28 & 0.16 & 0.98 \\
\hline & 173 & 0.11 & 20.5 & 9.21 & 1.99 & 0.56 & 0.28 & 0.16 & 1.05 \\
\hline & 197 & 0.13 & 20.1 & 9.29 & 1.94 & 0.48 & 0.33 & 0.19 & 1.04 \\
\hline & 221 & 0.15 & 19.7 & 8.99 & 1.84 & 0.47 & 0.33 & 0.21 & 0.98 \\
\hline & 289 & 0.19 & 18.9 & 9.55 & 2.34 & 0.34 & 0.39 & 0.27 & 1.15 \\
\hline 0.91 & 0 & 0.00 & 23.2 & 10.1 & & 0.94 & 0.06 & & 1.10 \\
\hline & 25 & 0.01 & 22.9 & 9.28 & & 0.90 & 0.10 & & 1.09 \\
\hline & 51 & 0.03 & 23.1 & 12.9 & 3.19 & 0.77 & 0.17 & 0.06 & 1.03 \\
\hline & 76 & 0.04 & 22.1 & 7.71 & 0.15 & 0.47 & 0.10 & 0.43 & 0.99 \\
\hline & 102 & 0.06 & 22.1 & 9.67 & 1.20 & 0.69 & 0.19 & 0.11 & 1.10 \\
\hline & 128 & 0.07 & 21.8 & 9.70 & 1.52 & 0.65 & 0.23 & 0.12 & 1.01 \\
\hline & 156 & 0.09 & 22.1 & 11.3 & 2.01 & 0.54 & 0.31 & 0.15 & 1.00 \\
\hline & 181 & 0.10 & 21.0 & 9.55 & 1.55 & 0.55 & 0.28 & 0.17 & 1.16 \\
\hline & 208 & 0.11 & 20.0 & 8.26 & 1.46 & 0.54 & 0.28 & 0.18 & 1.20 \\
\hline & 232 & 0.13 & 19.7 & 8.55 & 1.78 & 0.50 & 0.31 & 0.19 & 1.09 \\
\hline & 258 & 0.14 & 19.2 & 8.35 & 1.52 & 0.46 & 0.33 & 0.21 & 1.08 \\
\hline & 281 & 0.15 & 18.6 & 8.21 & 1.50 & 0.42 & 0.34 & 0.24 & 1.03 \\
\hline & 309 & 0.17 & 18.0 & 7.63 & 1.45 & 0.42 & 0.35 & 0.23 & 0.95 \\
\hline 1.06 & 0 & 0.00 & 23.1 & 10.3 & & 0.95 & 0.05 & & 1.01 \\
\hline & 43 & 0.02 & 22.7 & 8.77 & 0.14 & 0.59 & 0.08 & 0.03 & 1.03 \\
\hline & 64 & 0.03 & 22.6 & 9.33 & 0.83 & 0.81 & 0.13 & 0.06 & 1.02 \\
\hline & 105 & 0.05 & 22.6 & 10.7 & 1.56 & 0.75 & 0.18 & 0.07 & 1.04 \\
\hline & 149 & 0.07 & 21.8 & 8.92 & 1.44 & 0.73 & 0.19 & 0.09 & 0.99 \\
\hline & 198 & 0.09 & 21.4 & 8.68 & 1.16 & 0.63 & 0.24 & 0.12 & 1.11 \\
\hline & 211 & 0.10 & 21.5 & 9.91 & 1.87 & 0.59 & 0.28 & 0.14 & 1.01 \\
\hline & 243 & 0.11 & 21.0 & 10.1 & 1.94 & 0.53 & 0.31 & 0.17 & 1.05 \\
\hline & 276 & 0.13 & 20.2 & 9.37 & 1.75 & 0.49 & 0.31 & 0.21 & 1.04 \\
\hline & 309 & 0.15 & 19.3 & 7.89 & 1.32 & 0.50 & 0.30 & 0.20 & 1.17 \\
\hline
\end{tabular}




\begin{tabular}{|l|l|l|l|l|l|l|l|l|l|} 
& 339 & 0.16 & 18.6 & 8.32 & 1.89 & 0.44 & 0.24 & 0.33 & 1.11 \\
\cline { 2 - 11 } & 358 & 0.17 & 18.5 & 8.20 & 1.60 & 0.40 & 0.36 & 0.23 & 0.96 \\
\hline & 404 & 0.19 & 18.6 & 9.47 & 2.18 & 0.30 & 0.40 & 0.29 & 1.13 \\
\hline
\end{tabular}

Table 4a: FBM parameters retrieved by using Equation $\mathrm{S} 2$ to fit the fluorescence decays of DNA-EB quenched by $\mathrm{Cu}^{2+}$ cations with an $\mathrm{Na}_{2} \mathrm{SO}_{4}$ concentration of $5 \times 10^{-4} \mathrm{M}$.

\begin{tabular}{|c|c|c|c|c|c|c|c|c|c|}
\hline $\begin{array}{c}{[\mathrm{DNA}]} \\
\mathrm{mM} \text { of bp }\end{array}$ & {$\left[\mathrm{Cu}^{2+}\right](\mu \mathrm{M})$} & {$\left[\mathrm{Cu}^{2+}\right] /[\mathrm{P}]$} & $\begin{array}{l}k_{\mathrm{e}}[b l o b] \\
\times 10^{7} \mathrm{~s}^{-1}\end{array}$ & $\begin{aligned} & k_{\text {blob }} \\
& \times 10^{7} \mathrm{~s}^{-1} \\
&\end{aligned}$ & $<\mathrm{n}>$ & $\tau_{\text {fast }}$ & $a_{\text {fast }}$ & $a_{2}$ & $\chi^{2}$ \\
\hline \multirow[t]{6}{*}{0.30} & 20 & 0.03 & 0.00 & 3.10 & 0.16 & 1.27 & 0.04 & 0.96 & 1.08 \\
\hline & 40 & 0.07 & 0.51 & 3.75 & 0.35 & 1.28 & 0.09 & 0.91 & 1.04 \\
\hline & 59 & 0.10 & 1.08 & 3.62 & 1.30 & 1.69 & 0.24 & 0.76 & 1.20 \\
\hline & 79 & 0.13 & 0.87 & 2.93 & 2.05 & 1.78 & 0.31 & 0.69 & 1.18 \\
\hline & 100 & 0.17 & 1.68 & 4.18 & 2.45 & 1.40 & 0.32 & 0.68 & 1.07 \\
\hline & 115 & 0.19 & 1.71 & 4.14 & 2.75 & 1.34 & 0.33 & 0.67 & 1.08 \\
\hline \multirow[t]{5}{*}{0.45} & 30 & 0.03 & 0.37 & 4.38 & 0.19 & 0.00 & 0.39 & 0.61 & 1.33 \\
\hline & 60 & 0.07 & 0.81 & 4.32 & 0.60 & 1.67 & 0.17 & 0.83 & 1.08 \\
\hline & 90 & 0.10 & 1.05 & 3.95 & 1.24 & 1.26 & 0.24 & 0.76 & 1.13 \\
\hline & 119 & 0.13 & 1.44 & 4.09 & 1.91 & 1.40 & 0.29 & 0.71 & 1.15 \\
\hline & 151 & 0.17 & 1.77 & 4.45 & 2.40 & 1.27 & 0.31 & 0.69 & 1.19 \\
\hline \multirow[t]{6}{*}{0.76} & 50 & 0.03 & 0.12 & 2.74 & 0.21 & 1.29 & 0.07 & 0.93 & 1.13 \\
\hline & 99 & 0.07 & 0.96 & 4.12 & 0.65 & 1.40 & 0.16 & 0.84 & 1.06 \\
\hline & 147 & 0.10 & 1.23 & 4.25 & 1.09 & 1.57 & 0.21 & 0.79 & 1.22 \\
\hline & 201 & 0.13 & 1.45 & 4.12 & 1.79 & 1.32 & 0.29 & 0.71 & 1.15 \\
\hline & 251 & 0.17 & 1.67 & 4.21 & 2.25 & 1.29 & 0.31 & 0.69 & 1.14 \\
\hline & 287 & 0.19 & 2.18 & 4.90 & 2.59 & 1.17 & 0.31 & 0.69 & 1.23 \\
\hline \multirow[t]{3}{*}{1.06} & 139 & 0.07 & 0.62 & 3.22 & 0.68 & 2.08 & 0.15 & 0.85 & 1.15 \\
\hline & 208 & 0.10 & 1.12 & 3.84 & 1.09 & 1.60 & 0.22 & 0.78 & 1.08 \\
\hline & 280 & 0.13 & 1.44 & 3.95 & 1.72 & 1.51 & 0.28 & 0.72 & 1.13 \\
\hline
\end{tabular}




\begin{tabular}{|c|c|c|c|c|c|c|c|c|c|}
\hline & 352 & 0.17 & 1.82 & 4.33 & 2.35 & 1.33 & 0.31 & 0.69 & 1.09 \\
\hline \multirow[t]{4}{*}{1.36} & 180 & 0.07 & 1.03 & 4.64 & 0.64 & 1.41 & 0.16 & 0.84 & 1.11 \\
\hline & 271 & 0.10 & 1.30 & 4.54 & 1.11 & 1.42 & 0.21 & 0.79 & 1.17 \\
\hline & 361 & 0.13 & 1.65 & 4.57 & 1.61 & 1.41 & 0.26 & 0.74 & 1.13 \\
\hline & 447 & 0.16 & 1.96 & 4.69 & 2.51 & 1.19 & 0.32 & 0.68 & 1.12 \\
\hline
\end{tabular}

Table 4b: FBM parameters retrieved by using Equation $\mathrm{S} 2$ to fit the fluorescence decays of DNA-EB quenched by $\mathrm{Cu}^{2+}$ cations with an $\mathrm{Na}_{2} \mathrm{SO}_{4}$ concentration of $5 \times 10^{-3} \mathrm{M}$.

\begin{tabular}{|c|c|c|c|c|c|c|c|c|c|}
\hline $\begin{array}{c}{[\mathrm{DNA}]} \\
\mathrm{mM} \text { of } b p\end{array}$ & {$\left[\mathrm{Cu}^{2+}\right](\mu \mathrm{M})$} & {$\left[\mathrm{Cu}^{2+}\right] /[\mathrm{P}]$} & $\begin{array}{l}k_{\mathrm{e}}[\text { blob] } \\
\times 10^{7} \mathrm{~s}^{-1}\end{array}$ & $\begin{aligned} & k_{\text {blob }} \\
& \times 10^{7} \mathrm{~s}^{-1}\end{aligned}$ & $<\mathrm{n}>$ & $\tau_{\text {fast }}$ & $a_{\text {fast }}$ & $a_{2}$ & $\chi^{2}$ \\
\hline \multirow[t]{6}{*}{0.30} & 20 & 0.03 & 0.00 & 2.39 & 0.16 & 1.30 & 0.07 & 0.93 & 1.13 \\
\hline & 40 & 0.07 & 0.85 & 4.87 & 0.51 & 0.80 & 0.17 & 0.83 & 1.13 \\
\hline & 60 & 0.10 & 0.80 & 3.51 & 0.96 & 1.64 & 0.21 & 0.79 & 1.17 \\
\hline & 79 & 0.13 & 1.31 & 4.07 & 1.20 & 1.07 & 0.24 & 0.76 & 1.18 \\
\hline & 99 & 0.16 & 1.48 & 4.04 & 1.60 & 1.60 & 0.27 & 0.73 & 1.17 \\
\hline & 115 & 0.19 & 1.49 & 4.12 & 1.91 & 1.41 & 0.29 & 0.71 & 1.22 \\
\hline \multirow[t]{6}{*}{0.45} & 30 & 0.03 & 0.63 & 4.70 & 0.16 & 1.90 & 0.05 & 0.95 & 1.01 \\
\hline & 59 & 0.06 & 1.04 & 4.16 & 0.57 & 1.81 & 0.15 & 0.85 & 1.20 \\
\hline & 90 & 0.10 & 1.22 & 4.40 & 0.92 & 1.60 & 0.20 & 0.80 & 1.08 \\
\hline & 120 & 0.13 & 1.60 & 4.43 & 1.35 & 1.57 & 0.25 & 0.75 & 1.12 \\
\hline & 149 & 0.16 & 1.54 & 4.01 & 1.90 & 1.58 & 0.28 & 0.72 & 1.19 \\
\hline & 172 & 0.19 & 1.66 & 4.09 & 2.18 & 1.54 & 0.31 & 0.69 & 1.09 \\
\hline \multirow[t]{6}{*}{0.76} & 50 & 0.03 & 1.02 & 5.98 & 0.21 & 1.35 & 0.06 & 0.94 & 1.20 \\
\hline & 100 & 0.07 & 1.08 & 4.37 & 0.50 & 1.73 & 0.13 & 0.87 & 1.10 \\
\hline & 148 & 0.10 & 0.84 & 3.61 & 0.91 & 1.93 & 0.20 & 0.80 & 1.11 \\
\hline & 200 & 0.13 & 1.30 & 4.36 & 1.34 & 1.38 & 0.26 & 0.74 & 1.15 \\
\hline & 249 & 0.16 & 1.09 & 3.11 & 2.13 & 1.84 & 0.30 & 0.70 & 1.03 \\
\hline & 287 & 0.19 & 1.84 & 4.38 & 2.03 & 1.54 & 0.29 & 0.71 & 1.15 \\
\hline 1.06 & 138 & 0.07 & 0.63 & 2.83 & 0.63 & 2.66 & 0.14 & 0.86 & 1.19 \\
\hline
\end{tabular}




\begin{tabular}{|c|c|c|c|c|c|c|c|c|c|}
\hline & 208 & 0.10 & 1.06 & 4.32 & 0.93 & 1.35 & 0.19 & 0.81 & 1.26 \\
\hline & 280 & 0.13 & 1.39 & 4.00 & 1.52 & 1.59 & 0.26 & 0.74 & 1.14 \\
\hline & 347 & 0.16 & 1.73 & 4.05 & 2.27 & 1.51 & 0.33 & 0.67 & 1.18 \\
\hline & 399 & 0.19 & 2.04 & 4.45 & 2.70 & 1.30 & 0.33 & 0.67 & 1.25 \\
\hline \multirow[t]{5}{*}{1.36} & 179 & 0.07 & 0.83 & 4.78 & 0.49 & 1.59 & 0.13 & 0.87 & 1.10 \\
\hline & 269 & 0.10 & 1.08 & 4.81 & 0.86 & 1.24 & 0.20 & 0.80 & 1.14 \\
\hline & 349 & 0.13 & 1.29 & 3.81 & 1.58 & 1.68 & 0.28 & 0.72 & 1.11 \\
\hline & 455 & 0.17 & 1.43 & 3.90 & 2.20 & 1.47 & 0.31 & 0.69 & 1.12 \\
\hline & 505 & 0.19 & 2.01 & 4.26 & 2.44 & 1.57 & 0.32 & 0.68 & 1.15 \\
\hline
\end{tabular}

Table 4c: FBM parameters retrieved by using Equation $\mathrm{S} 2$ to fit the fluorescence decays of DNA-EB quenched by $\mathrm{Cu}^{2+}$ cations with an $\mathrm{Na}_{2} \mathrm{SO}_{4}$ concentration of $7.5 \times 10^{-3} \mathrm{M}$.

\begin{tabular}{|c|c|c|c|c|c|c|c|c|c|}
\hline $\begin{array}{c}{[\text { DNA] }} \\
\mathrm{mM} \text { of bp }\end{array}$ & {$\left[\mathrm{Cu}^{2+}\right](\mu \mathrm{M})$} & {$\left[\mathrm{Cu}^{2+}\right] /[\mathrm{P}]$} & $\begin{array}{l}k_{\mathrm{e}}[\text { blob }] \\
\times 10^{7} \mathrm{~s}^{-1}\end{array}$ & $\begin{aligned} & k_{\text {blob }} \\
\times & 10^{7} \mathrm{~s}^{-1}\end{aligned}$ & $<\mathrm{n}>$ & $\tau_{\text {fast }}$ & $a_{\text {fast }}$ & $a_{2}$ & $\chi^{2}$ \\
\hline \multirow{9}{*}{0.30} & 10 & 0.02 & 0.96 & 5.37 & 0.15 & 0.58 & 0.09 & 0.91 & 1.00 \\
\hline & 22 & 0.04 & 1.07 & 4.32 & 0.33 & 1.34 & 0.10 & 0.90 & 1.17 \\
\hline & 31 & 0.05 & 0.95 & 3.50 & 0.53 & 1.76 & 0.14 & 0.86 & 1.12 \\
\hline & 40 & 0.07 & 1.10 & 4.63 & 0.63 & 1.40 & 0.15 & 0.85 & 1.03 \\
\hline & 54 & 0.09 & 1.01 & 3.60 & 1.03 & 1.71 & 0.22 & 0.78 & 0.96 \\
\hline & 60 & 0.10 & 1.45 & 5.11 & 0.90 & 1.09 & 0.23 & 0.77 & 1.22 \\
\hline & 77 & 0.13 & 1.18 & 3.94 & 1.12 & 1.72 & 0.23 & 0.77 & 0.97 \\
\hline & 101 & 0.17 & 1.32 & 4.55 & 1.55 & 1.18 & 0.28 & 0.72 & 1.41 \\
\hline & 112 & 0.19 & 1.27 & 3.66 & 1.79 & 1.90 & 0.27 & 0.73 & 1.13 \\
\hline \multirow[t]{4}{*}{0.45} & 16 & 0.01 & 0.58 & 3.08 & 0.16 & 1.35 & 0.05 & 0.95 & 1.11 \\
\hline & 30 & 0.03 & 0.33 & 2.63 & 0.33 & 2.64 & 0.08 & 0.92 & 0.97 \\
\hline & 48 & 0.02 & 0.26 & 1.95 & 0.63 & 0.31 & 0.15 & 0.85 & 1.10 \\
\hline & 62 & 0.07 & 0.79 & 3.65 & 0.62 & 1.85 & 0.15 & 0.85 & 1.12 \\
\hline
\end{tabular}




\begin{tabular}{|c|c|c|c|c|c|c|c|c|c|}
\hline & 79 & 0.03 & 0.88 & 3.67 & 0.78 & 1.83 & 0.18 & 0.82 & 0.93 \\
\hline & 89 & 0.10 & 1.17 & 4.45 & 0.83 & 1.35 & 0.21 & 0.79 & 1.06 \\
\hline & 118 & 0.05 & 1.03 & 3.63 & 1.24 & 1.65 & 0.24 & 0.76 & 1.10 \\
\hline & 155 & 0.17 & 1.28 & 3.91 & 1.63 & 1.76 & 0.26 & 0.74 & 1.09 \\
\hline & 171 & 0.19 & 1.36 & 3.92 & 1.80 & 1.50 & 0.31 & 0.69 & 1.16 \\
\hline \multirow[t]{9}{*}{0.76} & 27 & 0.02 & 0.15 & 2.43 & 0.15 & 1.09 & 0.05 & 0.95 & 1.23 \\
\hline & 50 & 0.03 & 0.56 & 3.56 & 0.28 & 1.64 & 0.07 & 0.93 & 1.14 \\
\hline & 78 & 0.05 & 1.13 & 6.05 & 0.38 & 0.46 & 0.22 & 0.78 & 1.15 \\
\hline & 99 & 0.07 & 0.77 & 2.98 & 0.65 & 2.05 & 0.17 & 0.83 & 1.05 \\
\hline & 134 & 0.09 & 0.99 & 4.02 & 0.84 & 1.52 & 0.18 & 0.82 & 1.04 \\
\hline & 154 & 0.10 & 1.04 & 3.79 & 0.97 & 1.67 & 0.21 & 0.79 & 1.08 \\
\hline & 195 & 0.13 & 1.56 & 4.67 & 1.20 & 1.40 & 0.23 & 0.77 & 1.09 \\
\hline & 257 & 0.17 & 1.56 & 4.16 & 1.84 & 1.68 & 0.28 & 0.72 & 1.04 \\
\hline & 280 & 0.19 & 1.83 & 4.94 & 1.87 & 1.24 & 0.27 & 0.73 & 1.18 \\
\hline \multirow[t]{9}{*}{1.06} & 36 & 0.02 & 0.00 & 3.26 & 0.15 & 0.33 & 0.06 & 0.94 & 1.14 \\
\hline & 72 & 0.03 & 0.59 & 4.21 & 0.16 & 2.62 & 0.06 & 0.94 & 1.09 \\
\hline & 110 & 0.05 & 0.52 & 3.12 & 0.46 & 2.33 & 0.11 & 0.89 & 1.09 \\
\hline & 141 & 0.07 & 0.47 & 3.24 & 0.57 & 1.78 & 0.14 & 0.86 & 1.06 \\
\hline & 187 & 0.09 & 0.97 & 4.26 & 0.76 & 1.45 & 0.17 & 0.83 & 1.14 \\
\hline & 211 & 0.10 & 1.11 & 3.94 & 0.95 & 1.91 & 0.20 & 0.80 & 1.15 \\
\hline & 274 & 0.13 & 1.19 & 4.01 & 1.37 & 1.49 & 0.25 & 0.75 & 1.12 \\
\hline & 352 & 0.17 & 1.52 & 4.02 & 1.93 & 1.58 & 0.28 & 0.72 & 1.18 \\
\hline & 392 & 0.18 & 1.60 & 4.00 & 2.17 & 1.62 & 0.29 & 0.71 & 1.26 \\
\hline \multirow[t]{6}{*}{1.36} & 44 & 0.02 & 0.44 & 4.72 & 0.11 & 0.77 & 0.06 & 0.94 & 1.16 \\
\hline & 89 & 0.03 & 0.58 & 3.78 & 0.24 & 1.31 & 0.09 & 0.91 & 1.21 \\
\hline & 138 & 0.05 & 0.73 & 3.76 & 0.39 & 1.75 & 0.11 & 0.89 & 1.07 \\
\hline & 180 & 0.07 & 0.47 & 2.80 & 0.65 & 2.45 & 0.15 & 0.85 & 1.06 \\
\hline & 243 & 0.09 & 0.96 & 3.91 & 0.79 & 1.60 & 0.19 & 0.81 & 1.19 \\
\hline & 272 & 0.10 & 0.76 & 3.03 & 1.03 & 1.96 & 0.22 & 0.78 & 1.23 \\
\hline
\end{tabular}




\begin{tabular}{|l|l|l|l|l|l|l|l|l|l|l|l|l|l|l|}
\hline & 354 & 0.13 & 1.32 & 4.21 & 1.31 & 1.66 & 0.23 & 0.77 & 1.18 & 1.48 & 0.30 & 0.70 & 1.12 \\
\cline { 2 - 8 } & 455 & 0.17 & 1.66 & 4.17 & 1.94 & 1.48 & \\
\hline
\end{tabular}

Table 4d: FBM parameters retrieved by using Equation $\mathrm{S} 2$ to fit the fluorescence decays of DNA-EB quenched by $\mathrm{Cu}^{2+}$ cations with an $\mathrm{Na}_{2} \mathrm{SO}_{4}$ concentration of $1.25 \times 10^{-2} \mathrm{M}$.

\begin{tabular}{|c|c|c|c|c|c|c|c|c|c|}
\hline $\begin{array}{c}\text { [DNA] } \\
\mathrm{mM} \text { of } \mathrm{bp}\end{array}$ & {$\left[\mathrm{Cu}^{2+}\right](\mu \mathrm{M})$} & {$\left[\mathrm{Cu}^{2+}\right] /[\mathrm{P}]$} & $\begin{array}{l}k_{\mathrm{e}}[b l o b] \\
\times 10^{7} \mathrm{~s}^{-1}\end{array}$ & $\begin{aligned} & k_{\text {blob }} \\
\times & 10^{7} \mathrm{~s}^{-1}\end{aligned}$ & $<\mathrm{n}>$ & $\tau_{\text {fast }}$ & $a_{f a s t}$ & $a_{2}$ & $\chi^{2}$ \\
\hline \multirow[t]{9}{*}{0.30} & 10 & 0.02 & 0.79 & 5.94 & 0.15 & 0.37 & 0.16 & 0.84 & 0.93 \\
\hline & 22 & 0.04 & 0.92 & 4.25 & 0.31 & 1.64 & 0.09 & 0.91 & 1.29 \\
\hline & 31 & 0.05 & 0.91 & 3.86 & 0.48 & 1.50 & 0.13 & 0.87 & 1.05 \\
\hline & 40 & 0.07 & 0.79 & 3.80 & 0.58 & 1.40 & 0.15 & 0.85 & 1.07 \\
\hline & 54 & 0.09 & 1.00 & 3.81 & 0.74 & 1.68 & 0.18 & 0.82 & 1.15 \\
\hline & 60 & 0.10 & 1.17 & 3.80 & 0.81 & 1.96 & 0.18 & 0.82 & 1.23 \\
\hline & 77 & 0.13 & 1.24 & 4.42 & 0.92 & 1.47 & 0.20 & 0.80 & 1.26 \\
\hline & 101 & 0.17 & 1.56 & 5.15 & 1.05 & 1.23 & 0.22 & 0.78 & 1.16 \\
\hline & 112 & 0.19 & 1.37 & 4.74 & 1.21 & 1.21 & 0.24 & 0.76 & 1.29 \\
\hline \multirow[t]{9}{*}{0.45} & 15 & 0.02 & 0.25 & 2.49 & 0.18 & 0.39 & 0.13 & 0.87 & 1.04 \\
\hline & 32 & 0.04 & 0.57 & 3.09 & 0.32 & 1.63 & 0.09 & 0.91 & 1.15 \\
\hline & 46 & 0.05 & 1.08 & 5.19 & 0.41 & 0.93 & 0.12 & 0.88 & 1.18 \\
\hline & 58 & 0.06 & 0.93 & 4.06 & 0.55 & 1.41 & 0.14 & 0.86 & 1.14 \\
\hline & 80 & 0.09 & 1.02 & 3.67 & 0.78 & 1.90 & 0.17 & 0.83 & 1.10 \\
\hline & 90 & 0.10 & 1.03 & 3.34 & 0.87 & 1.75 & 0.19 & 0.81 & 1.14 \\
\hline & 118 & 0.13 & 1.07 & 3.62 & 1.08 & 1.76 & 0.21 & 0.79 & 1.11 \\
\hline & 153 & 0.17 & 1.23 & 3.85 & 1.34 & 1.59 & 0.24 & 0.76 & 1.20 \\
\hline & 166 & 0.18 & 1.55 & 4.58 & 1.32 & 1.41 & 0.26 & 0.74 & 1.12 \\
\hline \multirow[t]{2}{*}{0.76} & 51 & 0.03 & 0.57 & 3.21 & 0.27 & 2.37 & 0.07 & 0.93 & 1.06 \\
\hline & 77 & 0.05 & 0.72 & 3.63 & 0.37 & 1.75 & 0.11 & 0.89 & 0.99 \\
\hline
\end{tabular}




\begin{tabular}{|c|c|c|c|c|c|c|c|c|c|}
\hline & 99 & 0.07 & 0.82 & 4.17 & 0.50 & 1.74 & 0.12 & 0.88 & 1.17 \\
\hline & 135 & 0.09 & 1.14 & 4.50 & 0.68 & 1.45 & 0.17 & 0.83 & 1.03 \\
\hline & 148 & 0.10 & 1.02 & 3.98 & 0.77 & 1.68 & 0.19 & 0.81 & 0.99 \\
\hline & 192 & 0.13 & 1.06 & 4.04 & 1.30 & 1.59 & 0.21 & 0.79 & 1.10 \\
\hline & 252 & 0.17 & 1.24 & 3.70 & 1.42 & 1.69 & 0.27 & 0.73 & 1.19 \\
\hline & 278 & 0.18 & 1.28 & 3.59 & 1.65 & 1.78 & 0.27 & 0.73 & 1.22 \\
\hline \multirow[t]{9}{*}{1.06} & 36 & 0.02 & 0.51 & 3.07 & 0.13 & 1.52 & 0.06 & 0.94 & 1.11 \\
\hline & 70 & 0.03 & 0.60 & 3.43 & 0.26 & 1.70 & 0.10 & 0.91 & 1.11 \\
\hline & 110 & 0.05 & 0.65 & 3.70 & 0.47 & 1.29 & 0.13 & 0.87 & 1.08 \\
\hline & 138 & 0.07 & 0.87 & 3.56 & 0.54 & 2.08 & 0.13 & 0.87 & 1.09 \\
\hline & 185 & 0.09 & 1.07 & 4.66 & 0.75 & 1.18 & 0.17 & 0.83 & 1.18 \\
\hline & 210 & 0.10 & 1.11 & 3.77 & 0.93 & 1.93 & 0.20 & 0.80 & 1.20 \\
\hline & 274 & 0.13 & 1.20 & 3.90 & 1.25 & 1.56 & 0.23 & 0.77 & 1.20 \\
\hline & 356 & 0.17 & 1.71 & 5.13 & 1.49 & 1.10 & 0.27 & 0.73 & 1.26 \\
\hline & 391 & 0.18 & 1.55 & 4.37 & 1.76 & 1.29 & 0.28 & 0.72 & 1.24 \\
\hline \multirow[t]{9}{*}{1.36} & 43 & 0.02 & 0.01 & 3.24 & 0.11 & 0.74 & 0.05 & 0.95 & 1.02 \\
\hline & 89 & 0.03 & 0.26 & 3.64 & 0.25 & 0.87 & 0.08 & 0.92 & 0.99 \\
\hline & 139 & 0.05 & 0.58 & 3.37 & 0.39 & 1.77 & 0.11 & 0.89 & 1.14 \\
\hline & 179 & 0.07 & 0.77 & 3.66 & 0.48 & 1.79 & 0.12 & 0.88 & 1.14 \\
\hline & 243 & 0.09 & 0.73 & 3.23 & 0.77 & 1.86 & 0.18 & 0.82 & 1.03 \\
\hline & 271 & 0.10 & 1.02 & 3.80 & 0.80 & 1.65 & 0.19 & 0.81 & 1.03 \\
\hline & 349 & 0.13 & 1.25 & 4.43 & 1.10 & 1.13 & 0.24 & 0.76 & 1.19 \\
\hline & 455 & 0.17 & 1.44 & 4.23 & 1.53 & 1.25 & 0.28 & 0.72 & 1.19 \\
\hline & 503 & 0.18 & 1.80 & 4.71 & 1.62 & 1.35 & 0.27 & 0.73 & 1.15 \\
\hline
\end{tabular}

Table 4e: FBM parameters retrieved by using Equation $\mathrm{S} 2$ to fit the fluorescence decays of DNA-EB quenched by $\mathrm{Cu}^{2+}$ cations with an $\mathrm{Na}_{2} \mathrm{SO}_{4}$ concentration of $2.1 \times 10^{-2} \mathrm{M}$. 


\begin{tabular}{|c|c|c|c|c|c|c|c|c|c|}
\hline $\begin{array}{c}\text { [DNA] } \\
\mathrm{mM} \text { of bp }\end{array}$ & {$\left[\mathrm{Cu}^{2+}\right](\mu \mathrm{M})$} & {$\left[\mathrm{Cu}^{2+}\right] /[\mathrm{P}]$} & $\begin{array}{l}k_{\mathrm{e}}[b l o b] \\
\times 10^{7} \mathrm{~s}^{-1}\end{array}$ & $\begin{aligned} & k_{\text {blob }} \\
\times & 10^{7} \mathrm{~s}^{-1}\end{aligned}$ & $<\mathrm{n}>$ & $\tau_{\text {fast }}$ & $a_{\text {fast }}$ & $a_{2}$ & $\chi^{2}$ \\
\hline \multirow[t]{7}{*}{0.30} & 11 & 0.02 & 0.64 & 4.56 & 0.13 & 0.49 & 0.06 & 0.94 & 1.01 \\
\hline & 32 & 0.05 & 0.81 & 3.76 & 0.35 & 1.55 & 0.08 & 0.92 & 1.08 \\
\hline & 39 & 0.06 & 0.71 & 3.43 & 0.42 & 1.54 & 0.12 & 0.88 & 1.10 \\
\hline & 54 & 0.09 & 0.75 & 3.45 & 0.54 & 1.70 & 0.14 & 0.86 & 1.03 \\
\hline & 61 & 0.10 & 0.97 & 3.63 & 0.56 & 1.69 & 0.15 & 0.85 & 1.03 \\
\hline & 77 & 0.13 & 0.94 & 3.72 & 0.67 & 1.68 & 0.16 & 0.84 & 1.21 \\
\hline & 111 & 0.18 & 1.12 & 4.57 & 0.82 & 1.04 & 0.20 & 0.80 & 1.06 \\
\hline \multirow{6}{*}{0.45} & 30 & 0.03 & 0.75 & 6.29 & 0.16 & 0.36 & 0.11 & 0.89 & 1.12 \\
\hline & 59 & 0.06 & 0.53 & 3.46 & 0.34 & 1.31 & 0.12 & 0.88 & 1.12 \\
\hline & 89 & 0.10 & 0.61 & 3.37 & 0.51 & 2.09 & 0.13 & 0.87 & 1.08 \\
\hline & 121 & 0.13 & 1.06 & 5.24 & 0.55 & 1.09 & 0.15 & 0.85 & 1.08 \\
\hline & 151 & 0.17 & 0.62 & 3.26 & 0.83 & 1.74 & 0.18 & 0.82 & 1.15 \\
\hline & 173 & 0.19 & 0.92 & 3.53 & 0.90 & 1.85 & 0.19 & 0.81 & 1.12 \\
\hline \multirow[t]{6}{*}{0.76} & 25 & 0.02 & 0.00 & 3.52 & 0.08 & 1.55 & 0.02 & 0.98 & 1.13 \\
\hline & 50 & 0.03 & 0.00 & 2.31 & 0.02 & 0.11 & 0.27 & 0.73 & 1.15 \\
\hline & 101 & 0.07 & 0.57 & 4.98 & 0.31 & 0.87 & 0.11 & 0.89 & 1.19 \\
\hline & 150 & 0.10 & 0.46 & 3.05 & 0.55 & 2.29 & 0.14 & 0.86 & 1.17 \\
\hline & 199 & 0.13 & 0.77 & 3.43 & 0.79 & 2.06 & 0.16 & 0.84 & 1.20 \\
\hline & 245 & 0.16 & 0.76 & 3.17 & 1.04 & 1.83 & 0.22 & 0.78 & 1.15 \\
\hline \multirow[t]{7}{*}{1.06} & 35 & 0.02 & 0.85 & 3.77 & 0.09 & 0.59 & 0.09 & 0.91 & 1.03 \\
\hline & 69 & 0.03 & 0.70 & 5.78 & 0.18 & 0.19 & 0.39 & 0.61 & 1.04 \\
\hline & 140 & 0.07 & 0.79 & 4.42 & 0.38 & 1.18 & 0.13 & 0.87 & 0.96 \\
\hline & 211 & 0.10 & 1.05 & 4.34 & 0.63 & 1.56 & 0.16 & 0.84 & 1.00 \\
\hline & 282 & 0.13 & 1.20 & 4.08 & 0.91 & 1.55 & 0.21 & 0.79 & 0.98 \\
\hline & 345 & 0.16 & 1.32 & 4.19 & 1.12 & 1.55 & 0.22 & 0.78 & 1.02 \\
\hline & 397 & 0.19 & 1.40 & 4.10 & 1.27 & 1.49 & 0.23 & 0.77 & 1.15 \\
\hline \multirow[t]{3}{*}{1.36} & 22 & 0.01 & 0.00 & 3.51 & 0.03 & 0.09 & 0.64 & 0.36 & 1.02 \\
\hline & 45 & 0.02 & 0.64 & 8.00 & 0.07 & 1.74 & 0.00 & 1.00 & 0.94 \\
\hline & 88 & 0.03 & 0.37 & 5.00 & 0.15 & 0.55 & 0.11 & 0.89 & 1.01 \\
\hline
\end{tabular}




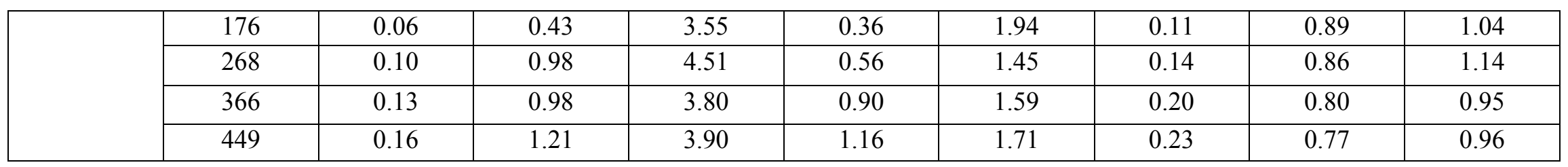

Table 4f: FBM parameters retrieved by using Equation $\mathrm{S} 2$ to fit the fluorescence decays of DNA-EB quenched by $\mathrm{Cu}^{2+}$ cations with an $\mathrm{Na}_{2} \mathrm{SO}_{4}$ concentration of $3 \times 10^{-2} \mathrm{M}$.

\begin{tabular}{|c|c|c|c|c|c|c|c|c|c|}
\hline $\begin{array}{c}{[\mathrm{DNA}]} \\
\mathrm{mM} \text { of bp }\end{array}$ & {$\left[\mathrm{Cu}^{2+}\right](\mu \mathrm{M})$} & {$\left[\mathrm{Cu}^{2+}\right] /[\mathrm{P}]$} & $\begin{array}{l}k_{\mathrm{e}}[b l o b] \\
\times 10^{7} \mathrm{~s}^{-1}\end{array}$ & $\begin{aligned} & k_{\text {blob }} \\
& \times 10^{7} \mathrm{~s}^{-1}\end{aligned}$ & $<\mathrm{n}>$ & $\tau_{\text {fast }}$ & $a_{\text {fast }}$ & $a_{2}$ & $\chi^{2}$ \\
\hline \multirow[t]{7}{*}{0.30} & 21 & 0.03 & 0.80 & 6.20 & 0.16 & 0.17 & 0.36 & 0.64 & 1.12 \\
\hline & 32 & 0.05 & 0.85 & 5.40 & 0.22 & 0.79 & 0.11 & 0.89 & 1.08 \\
\hline & 41 & 0.07 & 0.92 & 4.95 & 0.26 & 1.58 & 0.08 & 0.92 & 1.08 \\
\hline & 54 & 0.09 & 0.25 & 2.17 & 0.41 & 2.69 & 0.13 & 0.87 & 1.15 \\
\hline & 59 & 0.10 & 0.66 & 2.98 & 0.39 & 2.03 & 0.11 & 0.89 & 1.03 \\
\hline & 81 & 0.13 & 0.63 & 3.30 & 0.45 & 2.10 & 0.13 & 0.87 & 1.04 \\
\hline & 101 & 0.17 & 0.81 & 3.15 & 0.58 & 2.37 & 0.16 & 0.84 & 0.96 \\
\hline \multirow[t]{8}{*}{0.45} & 16 & 0.02 & 0.80 & 3.59 & 0.09 & 1.65 & 0.04 & 0.96 & 1.15 \\
\hline & 32 & 0.03 & 0.71 & 5.20 & 0.17 & 0.81 & 0.07 & 0.93 & 1.26 \\
\hline & 46 & 0.05 & 0.26 & 2.51 & 0.30 & 2.36 & 0.09 & 0.91 & 1.06 \\
\hline & 56 & 0.06 & 0.93 & 5.96 & 0.28 & 0.68 & 0.12 & 0.88 & 1.22 \\
\hline & 80 & 0.09 & 0.93 & 3.99 & 0.38 & 1.85 & 0.12 & 0.88 & 1.12 \\
\hline & 91 & 0.10 & 0.42 & 2.72 & 0.51 & 1.82 & 0.16 & 0.84 & 1.12 \\
\hline & 151 & 0.17 & 0.93 & 3.31 & 0.67 & 1.92 & 0.18 & 0.82 & 1.08 \\
\hline & 172 & 0.19 & 0.93 & 3.34 & 0.74 & 1.99 & 0.18 & 0.82 & 1.15 \\
\hline \multirow[t]{4}{*}{0.61} & 19 & 0.02 & 0.18 & 1.95 & 0.12 & 2.00 & 0.03 & 0.97 & 1.13 \\
\hline & 41 & 0.03 & 0.92 & 3.91 & 0.18 & 1.19 & 0.08 & 0.92 & 1.15 \\
\hline & 63 & 0.05 & 0.39 & 2.14 & 0.32 & 2.44 & 0.10 & 0.90 & 1.19 \\
\hline & 81 & 0.07 & 0.90 & 3.32 & 0.36 & 2.23 & 0.11 & 0.89 & 1.02 \\
\hline
\end{tabular}




\begin{tabular}{|c|c|c|c|c|c|c|c|c|c|}
\hline & 107 & 0.09 & 0.95 & 4.08 & 0.41 & 1.47 & 0.13 & 0.87 & 0.94 \\
\hline & 120 & 0.10 & 1.09 & 5.28 & 0.41 & 0.80 & 0.17 & 0.83 & 1.01 \\
\hline & 155 & 0.13 & 1.49 & 6.92 & 0.47 & 0.95 & 0.15 & 0.85 & 1.04 \\
\hline & 202 & 0.17 & 1.30 & 4.02 & 0.66 & 1.94 & 0.17 & 0.83 & 1.14 \\
\hline & 224 & 0.19 & 1.37 & 4.80 & 0.69 & 1.36 & 0.19 & 0.81 & 1.23 \\
\hline \multirow[t]{9}{*}{0.76} & 25 & 0.02 & 0.00 & 1.55 & 0.11 & 4.39 & 0.04 & 0.96 & 1.03 \\
\hline & 50 & 0.03 & 0.41 & 3.72 & 0.18 & 0.81 & 0.09 & 0.91 & 1.04 \\
\hline & 80 & 0.05 & 1.08 & 6.86 & 0.20 & 0.87 & 0.08 & 0.92 & 0.96 \\
\hline & 103 & 0.07 & 1.41 & 9.44 & 0.25 & 0.07 & 0.91 & 0.09 & 1.00 \\
\hline & 138 & 0.09 & 1.07 & 5.30 & 0.35 & 1.15 & 0.11 & 0.89 & 1.02 \\
\hline & 150 & 0.10 & 1.26 & 6.82 & 0.38 & 0.74 & 0.14 & 0.86 & 0.94 \\
\hline & 196 & 0.13 & 1.15 & 4.93 & 0.50 & 1.28 & 0.15 & 0.85 & 1.01 \\
\hline & 254 & 0.17 & 1.22 & 4.62 & 0.65 & 1.39 & 0.18 & 0.82 & 1.04 \\
\hline & 281 & 0.19 & 1.24 & 4.38 & 0.72 & 1.54 & 0.19 & 0.81 & 1.21 \\
\hline \multirow[t]{8}{*}{0.91} & 30 & 0.02 & 0.16 & 3.03 & 0.12 & 2.47 & 0.05 & 0.95 & 1.01 \\
\hline & 60 & 0.03 & 0.31 & 3.21 & 0.21 & 2.22 & 0.06 & 0.94 & 0.96 \\
\hline & 92 & 0.05 & 0.94 & 6.13 & 0.27 & 0.41 & 0.20 & 0.80 & 1.08 \\
\hline & 120 & 0.07 & 0.70 & 3.66 & 0.39 & 2.12 & 0.12 & 0.88 & 1.10 \\
\hline & 161 & 0.09 & 0.91 & 4.45 & 0.46 & 1.73 & 0.13 & 0.87 & 1.03 \\
\hline & 232 & 0.13 & 1.25 & 4.82 & 0.71 & 1.31 & 0.19 & 0.81 & 1.04 \\
\hline & 302 & 0.17 & 1.14 & 4.18 & 0.82 & 1.49 & 0.20 & 0.80 & 1.14 \\
\hline & 336 & 0.18 & 1.35 & 4.96 & 0.84 & 1.25 & 0.19 & 0.81 & 0.95 \\
\hline \multirow[t]{8}{*}{1.06} & 37 & 0.02 & 0.00 & 3.36 & 0.10 & 0.80 & 0.06 & 0.94 & 1.12 \\
\hline & 71 & 0.03 & 0.11 & 2.95 & 0.23 & 0.51 & 0.14 & 0.86 & 1.05 \\
\hline & 108 & 0.05 & 0.00 & 2.05 & 0.33 & 2.20 & 0.10 & 0.90 & 1.11 \\
\hline & 140 & 0.07 & 0.62 & 2.50 & 0.36 & 2.77 & 0.12 & 0.88 & 1.09 \\
\hline & 188 & 0.09 & 0.60 & 2.79 & 0.50 & 2.48 & 0.13 & 0.87 & 1.01 \\
\hline & 189 & 0.09 & 0.50 & 2.58 & 0.63 & 2.24 & 0.16 & 0.84 & 1.21 \\
\hline & 278 & 0.13 & 0.96 & 3.16 & 0.76 & 2.20 & 0.19 & 0.81 & 1.04 \\
\hline & 386 & 0.18 & 1.16 & 3.53 & 1.05 & 1.70 & 0.23 & 0.77 & 1.20 \\
\hline
\end{tabular}




\begin{tabular}{|c|c|c|c|c|c|c|c|c|c|}
\hline \multirow[t]{8}{*}{1.21} & 41 & 0.02 & 0.00 & 2.01 & 0.12 & 3.34 & 0.04 & 0.96 & 1.01 \\
\hline & 81 & 0.03 & 0.49 & 4.02 & 0.20 & 1.22 & 0.07 & 0.93 & 1.06 \\
\hline & 128 & 0.05 & 1.05 & 6.48 & 0.24 & 0.38 & 0.17 & 0.83 & 1.15 \\
\hline & 160 & 0.07 & 0.51 & 3.75 & 0.37 & 1.87 & 0.10 & 0.90 & 1.03 \\
\hline & 215 & 0.09 & 1.08 & 5.24 & 0.40 & 1.24 & 0.16 & 0.84 & 1.17 \\
\hline & 237 & 0.10 & 0.51 & 3.03 & 0.55 & 1.97 & 0.16 & 0.84 & 1.21 \\
\hline & 309 & 0.13 & 1.20 & 5.32 & 0.64 & 1.20 & 0.16 & 0.84 & 1.19 \\
\hline & 398 & 0.16 & 0.94 & 3.34 & 0.99 & 1.78 & 0.22 & 0.78 & 1.15 \\
\hline \multirow[t]{8}{*}{1.36} & 43 & 0.02 & 0.24 & 4.38 & 0.08 & 0.19 & 0.33 & 0.67 & 1.01 \\
\hline & 89 & 0.03 & 0.00 & 2.27 & 0.19 & 2.29 & 0.07 & 0.93 & 0.96 \\
\hline & 140 & 0.05 & 0.59 & 4.36 & 0.24 & 0.88 & 0.13 & 0.87 & 1.08 \\
\hline & 182 & 0.07 & 0.52 & 3.49 & 0.34 & 1.91 & 0.10 & 0.90 & 1.06 \\
\hline & 241 & 0.09 & 0.88 & 5.04 & 0.39 & 1.04 & 0.13 & 0.87 & 1.15 \\
\hline & 270 & 0.10 & 1.01 & 4.40 & 0.52 & 1.30 & 0.16 & 0.84 & 1.19 \\
\hline & 359 & 0.13 & 1.19 & 4.94 & 0.67 & 0.97 & 0.20 & 0.80 & 1.15 \\
\hline & 488 & 0.18 & 1.52 & 5.06 & 0.84 & 1.41 & 0.18 & 0.82 & 1.18 \\
\hline
\end{tabular}

Table 4g: FBM parameters retrieved by using Equation $\mathrm{S} 2$ to fit the fluorescence decays of DNA-EB quenched by $\mathrm{Cu}^{2+}$ cations with an $\mathrm{Na}_{2} \mathrm{SO}_{4}$ concentration of $4 \times 10^{-2} \mathrm{M}$.

\begin{tabular}{|c|c|c|c|c|c|c|c|c|c|}
\hline $\begin{array}{c}{[\mathrm{DNA}]} \\
\mathrm{mM} \text { of } \mathrm{bp}\end{array}$ & {$\left[\mathrm{Cu}^{2+}\right](\mu \mathrm{M})$} & {$\left[\mathrm{Cu}^{2+}\right] /[\mathrm{P}]$} & $\begin{array}{l}k_{\mathrm{e}}[\text { blob }] \\
\times 10^{7} \mathrm{~s}^{-1}\end{array}$ & $\begin{aligned} & k_{\text {blob }} \\
\times & 10^{7} \mathrm{~s}^{-1}\end{aligned}$ & $<\mathrm{n}>$ & $\tau_{\text {fast }}$ & $a_{\text {fast }}$ & $a_{2}$ & $\chi^{2}$ \\
\hline \multirow{5}{*}{0.30} & 20 & 0.03 & 0.00 & 3.49 & 0.01 & 0.15 & 0.31 & 0.69 & 1.03 \\
\hline & 59 & 0.10 & 0.60 & 3.70 & 0.27 & 2.28 & 0.08 & 0.92 & 1.12 \\
\hline & 80 & 0.13 & 0.49 & 2.78 & 0.39 & 2.17 & 0.11 & 0.89 & 1.14 \\
\hline & 100 & 0.17 & 0.50 & 2.43 & 0.47 & 2.57 & 0.13 & 0.87 & 1.04 \\
\hline & 116 & 0.19 & 0.93 & 3.99 & 0.43 & 2.01 & 0.11 & 0.89 & 1.10 \\
\hline \multirow[t]{4}{*}{0.45} & 30 & 0.03 & 0.67 & 5.12 & 0.10 & 1.25 & 0.05 & 0.95 & 1.09 \\
\hline & 60 & 0.07 & 0.94 & 5.37 & 0.18 & 1.17 & 0.09 & 0.91 & 1.13 \\
\hline & 90 & 0.10 & 0.00 & 1.99 & 0.40 & 2.72 & 0.12 & 0.88 & 1.09 \\
\hline & 120 & 0.13 & 0.82 & 4.80 & 0.36 & 1.37 & 0.12 & 0.88 & 1.13 \\
\hline
\end{tabular}




\begin{tabular}{|c|c|c|c|c|c|c|c|c|c|}
\hline & 151 & 0.17 & 1.15 & 6.33 & 0.40 & 1.00 & 0.13 & 0.87 & 1.19 \\
\hline & 173 & 0.19 & 0.65 & 3.50 & 0.54 & 1.74 & 0.16 & 0.84 & 1.09 \\
\hline \multirow[t]{6}{*}{0.61} & 39 & 0.03 & 0.23 & 3.48 & 0.07 & 2.59 & 0.04 & 0.96 & 1.09 \\
\hline & 80 & 0.07 & 0.73 & 5.76 & 0.17 & 0.40 & 0.13 & 0.87 & 1.19 \\
\hline & 119 & 0.10 & 0.69 & 5.55 & 0.24 & 0.83 & 0.09 & 0.91 & 1.06 \\
\hline & 160 & 0.13 & 1.07 & 7.06 & 0.29 & 0.22 & 0.37 & 0.63 & 1.02 \\
\hline & 201 & 0.17 & 0.96 & 4.83 & 0.38 & 1.79 & 0.11 & 0.89 & 1.13 \\
\hline & 230 & 0.19 & 0.82 & 3.57 & 0.57 & 1.92 & 0.16 & 0.84 & 1.18 \\
\hline \multirow[t]{6}{*}{0.76} & 24 & 0.02 & 0.00 & 4.94 & 0.08 & 1.33 & 0.01 & 0.99 & 1.14 \\
\hline & 101 & 0.07 & 0.32 & 4.36 & 0.16 & 0.87 & 0.08 & 0.92 & 1.10 \\
\hline & 152 & 0.10 & 0.66 & 4.20 & 0.36 & 2.05 & 0.09 & 0.91 & 1.16 \\
\hline & 203 & 0.13 & 0.99 & 5.45 & 0.49 & 1.14 & 0.16 & 0.84 & 1.07 \\
\hline & 259 & 0.17 & 1.00 & 4.27 & 0.61 & 1.88 & 0.15 & 0.85 & 1.11 \\
\hline & 287 & 0.19 & 0.93 & 3.62 & 0.75 & 1.94 & 0.18 & 0.82 & 1.06 \\
\hline \multirow[t]{7}{*}{0.91} & 30 & 0.02 & 0.00 & 3.36 & 0.06 & 0.25 & 0.18 & 0.82 & 1.21 \\
\hline & 60 & 0.03 & 0.33 & 3.10 & 0.13 & 1.27 & 0.07 & 0.93 & 1.10 \\
\hline & 119 & 0.07 & 1.03 & 4.54 & 0.21 & 1.89 & 0.08 & 0.92 & 0.97 \\
\hline & 180 & 0.10 & 1.28 & 6.17 & 0.30 & 1.25 & 0.09 & 0.91 & 1.19 \\
\hline & 231 & 0.13 & 0.88 & 2.91 & 0.56 & 2.47 & 0.17 & 0.83 & 1.17 \\
\hline & 301 & 0.17 & 0.93 & 3.45 & 0.67 & 2.03 & 0.18 & 0.82 & 1.12 \\
\hline & 343 & 0.19 & 0.88 & 3.10 & 0.80 & 2.23 & 0.19 & 0.81 & 1.11 \\
\hline \multirow[t]{7}{*}{1.06} & 35 & 0.02 & 0.00 & 3.70 & 0.06 & 0.10 & 0.37 & 0.63 & 1.17 \\
\hline & 71 & 0.03 & 0.64 & 7.58 & 0.10 & 0.17 & 0.39 & 0.61 & 1.08 \\
\hline & 140 & 0.07 & 0.47 & 3.54 & 0.23 & 1.48 & 0.08 & 0.92 & 1.15 \\
\hline & 210 & 0.10 & 0.67 & 4.75 & 0.34 & 1.28 & 0.12 & 0.88 & 1.06 \\
\hline & 280 & 0.13 & 1.06 & 4.42 & 0.56 & 1.52 & 0.16 & 0.84 & 1.09 \\
\hline & 357 & 0.17 & 1.35 & 5.38 & 0.60 & 1.41 & 0.15 & 0.85 & 1.06 \\
\hline & 398 & 0.19 & 1.26 & 4.94 & 0.72 & 1.33 & 0.18 & 0.82 & 1.09 \\
\hline \multirow[t]{3}{*}{1.21} & 79 & 0.03 & 0.52 & 5.40 & 0.14 & 0.87 & 0.07 & 0.93 & 1.10 \\
\hline & 160 & 0.07 & 0.13 & 2.47 & 0.34 & 2.56 & 0.10 & 0.90 & 1.12 \\
\hline & 239 & 0.10 & 0.79 & 4.31 & 0.40 & 1.75 & 0.11 & 0.89 & 0.99 \\
\hline
\end{tabular}




\begin{tabular}{|c|c|c|c|c|c|c|c|c|c|}
\hline & 320 & 0.13 & 1.10 & 4.50 & 0.61 & 1.65 & 0.17 & 0.83 & 1.15 \\
\hline & 404 & 0.17 & 1.32 & 5.15 & 0.73 & 1.37 & 0.16 & 0.84 & 1.00 \\
\hline & 468 & 0.19 & 1.41 & 4.31 & 0.84 & 1.73 & 0.20 & 0.80 & 1.15 \\
\hline \multirow[t]{6}{*}{1.36} & 45 & 0.02 & 0.00 & 3.81 & 0.06 & 0.52 & 0.06 & 0.94 & 1.00 \\
\hline & 181 & 0.07 & 0.00 & 1.67 & 0.32 & 3.15 & 0.09 & 0.91 & 1.01 \\
\hline & 279 & 0.10 & 0.92 & 4.47 & 0.39 & 1.39 & 0.13 & 0.87 & 1.22 \\
\hline & 362 & 0.13 & 1.02 & 4.06 & 0.60 & 1.77 & 0.15 & 0.85 & 1.01 \\
\hline & 446 & 0.16 & 1.38 & 4.92 & 0.67 & 1.37 & 0.18 & 0.82 & 1.06 \\
\hline & 515 & 0.19 & 1.25 & 4.31 & 0.79 & 1.53 & 0.20 & 0.80 & 1.06 \\
\hline
\end{tabular}

Table 5a: FBM parameters retrieved by using Equation $\mathrm{S} 2$ to fit the fluorescence decays of DNA-EB quenched by $\mathrm{Ni}^{2+}$ cations with an $\mathrm{Na}_{2} \mathrm{SO}_{4}$ concentration of $5 \times 10^{-4} \mathrm{M}$.

\begin{tabular}{|c|c|c|c|c|c|c|c|c|c|}
\hline $\begin{array}{c}{[\mathrm{DNA}]} \\
\mathrm{mM} \text { of } \mathrm{bp}\end{array}$ & {$\left[\mathrm{Ni}^{2+}\right](\mu \mathrm{M})$} & {$\left[\mathrm{Ni}^{2+}\right] /[\mathrm{P}]$} & $\begin{array}{l}k_{\mathrm{e}}[b l o b] \\
\times 10^{7} \mathrm{~s}^{-1} \\
\end{array}$ & $\begin{aligned} & k_{\text {blob }} \\
& \times 10^{7} \mathrm{~s}^{-1} \\
&\end{aligned}$ & $<\mathrm{n}>$ & $\tau_{\text {fast }}$ & $a_{\text {fast }}$ & $a_{2}$ & $\chi^{2}$ \\
\hline \multirow[t]{9}{*}{0.30} & 12 & 0.02 & 0.11 & 1.97 & 0.19 & 1.38 & 0.08 & 0.92 & 1.08 \\
\hline & 20 & 0.03 & 0.47 & 2.00 & 0.31 & 2.27 & 0.10 & 0.90 & 1.02 \\
\hline & 25 & 0.04 & 0.84 & 3.00 & 0.32 & 2.00 & 0.12 & 0.88 & 1.14 \\
\hline & 34 & 0.06 & 0.60 & 2.35 & 0.59 & 1.92 & 0.19 & 0.81 & 1.08 \\
\hline & 40 & 0.07 & 0.32 & 1.81 & 0.86 & 2.16 & 0.21 & 0.79 & 1.13 \\
\hline & 54 & 0.09 & 1.03 & 2.40 & 1.06 & 1.79 & 0.27 & 0.73 & 1.09 \\
\hline & 60 & 0.10 & 1.29 & 2.67 & 1.24 & 1.72 & 0.30 & 0.70 & 1.10 \\
\hline & 85 & 0.14 & 1.70 & 3.16 & 1.76 & 1.55 & 0.34 & 0.66 & 1.04 \\
\hline & 112 & 0.18 & 2.35 & 3.32 & 2.25 & 1.51 & 0.37 & 0.63 & 1.07 \\
\hline \multirow[t]{8}{*}{0.45} & 19 & 0.02 & 0.00 & 0.93 & 0.20 & 1.34 & 0.07 & 0.93 & 1.04 \\
\hline & 30 & 0.03 & 0.00 & 1.07 & 0.36 & 2.10 & 0.11 & 0.83 & 1.08 \\
\hline & 36 & 0.04 & 0.00 & 1.15 & 0.45 & 2.55 & 0.12 & 0.88 & 1.03 \\
\hline & 50 & 0.05 & 1.07 & 2.83 & 0.41 & 1.69 & 0.18 & 0.82 & 1.05 \\
\hline & 60 & 0.07 & 0.70 & 1.86 & 0.74 & 1.85 & 0.22 & 0.78 & 1.13 \\
\hline & 73 & 0.08 & 0.63 & 1.99 & 0.98 & 1.86 & 0.25 & 0.75 & 0.98 \\
\hline & 80 & 0.09 & 1.27 & 2.56 & 0.91 & 1.82 & 0.26 & 0.74 & 1.02 \\
\hline & 90 & 0.10 & 1.48 & 2.73 & 1.06 & 1.63 & 0.29 & 0.71 & 1.06 \\
\hline
\end{tabular}




\begin{tabular}{|c|c|c|c|c|c|c|c|c|c|}
\hline & 128 & 0.14 & 1.30 & 2.20 & 2.03 & 1.70 & 0.36 & 0.64 & 1.11 \\
\hline & 168 & 0.18 & 1.96 & 2.44 & 2.65 & 1.67 & 0.39 & 0.61 & 1.10 \\
\hline \multirow[t]{8}{*}{0.76} & 61 & 0.04 & 0.96 & 3.35 & 0.27 & 1.49 & 0.13 & 0.87 & 1.18 \\
\hline & 83 & 0.05 & 0.56 & 2.17 & 0.51 & 2.05 & 0.17 & 0.83 & 0.95 \\
\hline & 100 & 0.07 & 0.39 & 2.02 & 0.70 & 1.97 & 0.19 & 0.81 & 1.08 \\
\hline & 121 & 0.08 & 0.73 & 2.39 & 0.84 & 1.87 & 0.24 & 0.76 & 1.00 \\
\hline & 150 & 0.10 & 1.53 & 2.99 & 0.98 & 1.76 & 0.28 & 0.72 & 1.03 \\
\hline & 190 & 0.13 & 1.72 & 3.39 & 1.62 & 1.54 & 0.34 & 0.66 & 1.06 \\
\hline & 217 & 0.14 & 1.85 & 2.76 & 2.03 & 1.63 & 0.38 & 0.62 & 1.02 \\
\hline & 283 & 0.19 & 1.44 & 1.86 & 3.14 & 1.70 & 0.33 & 0.57 & 1.16 \\
\hline \multirow[t]{10}{*}{1.06} & 70 & 0.03 & 0.76 & 3.48 & 0.22 & 1.36 & 0.10 & 0.90 & 0.96 \\
\hline & 85 & 0.04 & 0.39 & 1.90 & 0.37 & 2.08 & 0.13 & 0.87 & 1.03 \\
\hline & 116 & 0.05 & 0.62 & 2.55 & 0.46 & 2.03 & 0.16 & 0.84 & 1.02 \\
\hline & 141 & 0.07 & 1.03 & 3.03 & 0.56 & 1.69 & 0.20 & 0.80 & 1.10 \\
\hline & 169 & 0.08 & 1.31 & 3.40 & 0.68 & 1.67 & 0.24 & 0.76 & 1.15 \\
\hline & 187 & 0.09 & 1.43 & 3.20 & 0.79 & 1.77 & 0.26 & 0.74 & 1.14 \\
\hline & 211 & 0.10 & 1.24 & 2.87 & 1.02 & 1.79 & 0.29 & 0.71 & 1.15 \\
\hline & 270 & 0.13 & 1.73 & 3.41 & 1.61 & 1.54 & 0.34 & 0.66 & 1.12 \\
\hline & 301 & 0.14 & 1.46 & 2.26 & 2.22 & 1.76 & 0.38 & 0.62 & 0.97 \\
\hline & 385 & 0.18 & 2.46 & 2.54 & 2.76 & 1.65 & 0.41 & 0.59 & 1.13 \\
\hline \multirow[t]{7}{*}{1.36} & 174 & 0.06 & 0.98 & 3.00 & 0.55 & 1.64 & 0.20 & 0.80 & 1.00 \\
\hline & 222 & 0.08 & 0.76 & 2.15 & 0.91 & 1.92 & 0.26 & 0.74 & 1.06 \\
\hline & 244 & 0.09 & 1.25 & 2.75 & 0.89 & 1.95 & 0.26 & 0.74 & 1.08 \\
\hline & 266 & 0.10 & 1.18 & 2.57 & 1.08 & 1.77 & 0.29 & 0.71 & 1.18 \\
\hline & 350 & 0.13 & 1.72 & 3.37 & 1.63 & 1.54 & 0.34 & 0.66 & 1.06 \\
\hline & 381 & 0.14 & 1.97 & 2.77 & 1.88 & 1.64 & 0.37 & 0.63 & 1.12 \\
\hline & 509 & 0.19 & 2.41 & 2.69 & 2.73 & 1.60 & 0.41 & 0.59 & 1.02 \\
\hline
\end{tabular}


Table 5b: FBM parameters retrieved by using Equation $\mathrm{S} 2$ to fit the fluorescence decays of DNA-EB quenched by $\mathrm{Ni}^{2+}$ cations with an $\mathrm{Na}_{2} \mathrm{SO}_{4}$ concentration of $5 \times 10^{-3} \mathrm{M}$.

\begin{tabular}{|c|c|c|c|c|c|c|c|c|c|}
\hline $\begin{array}{c}{[\mathrm{DNA}]} \\
\mathrm{mM} \text { of } \mathrm{bp}\end{array}$ & {$\left[\mathrm{Ni}^{2+}\right](\mu \mathrm{M})$} & {$\left[\mathrm{Ni}^{2+}\right] /[\mathrm{P}]$} & $\begin{array}{l}k_{\mathrm{e}}[b l o b] \\
\times 10^{7} \mathrm{~s}^{-1}\end{array}$ & $\begin{aligned} & k_{\text {blob }} \\
& \times 10^{7} \mathrm{~s}^{-1}\end{aligned}$ & $<\mathrm{n}>$ & $\tau_{\text {fast }}$ & $a_{\text {fast }}$ & $a_{2}$ & $\chi^{2}$ \\
\hline \multirow[t]{10}{*}{0.30} & 6 & 0.01 & 0.00 & 0.00 & 0.13 & 2.00 & 0.04 & 0.96 & 1.03 \\
\hline & 12 & 0.02 & 0.00 & 1.47 & 0.24 & 1.65 & 0.07 & 0.93 & 1.02 \\
\hline & 19 & 0.03 & 0.68 & 2.29 & 0.30 & 2.15 & 0.12 & 0.88 & 1.02 \\
\hline & 24 & 0.04 & 0.81 & 2.87 & 0.32 & 1.79 & 0.12 & 0.88 & 0.94 \\
\hline & 30 & 0.05 & 0.48 & 2.01 & 0.51 & 1.99 & 0.16 & 0.84 & 1.13 \\
\hline & 42 & 0.07 & 0.65 & 2.28 & 0.62 & 2.00 & 0.19 & 0.81 & 1.02 \\
\hline & 48 & 0.08 & 1.28 & 3.39 & 0.61 & 1.79 & 0.20 & 0.80 & 1.14 \\
\hline & 55 & 0.09 & 1.38 & 3.28 & 0.76 & 1.60 & 0.24 & 0.76 & 1.08 \\
\hline & 60 & 0.10 & 0.97 & 2.37 & 0.94 & 1.91 & 0.26 & 0.74 & 1.13 \\
\hline & 89 & 0.15 & 1.71 & 3.58 & 1.13 & 1.63 & 0.29 & 0.71 & 1.09 \\
\hline \multirow[t]{9}{*}{0.45} & 30 & 0.03 & 0.00 & 4.02 & 0.19 & 1.56 & 0.09 & 0.91 & 1.02 \\
\hline & 37 & 0.04 & 0.16 & 4.44 & 0.21 & 1.33 & 0.13 & 0.87 & 1.02 \\
\hline & 52 & 0.06 & 0.59 & 3.10 & 0.37 & 1.57 & 0.16 & 0.84 & 0.94 \\
\hline & 60 & 0.07 & 0.18 & 2.55 & 0.53 & 1.84 & 0.20 & 0.80 & 1.13 \\
\hline & 73 & 0.08 & 0.91 & 3.58 & 0.58 & 1.75 & 0.22 & 0.78 & 1.23 \\
\hline & 80 & 0.09 & 0.97 & 2.91 & 0.77 & 1.84 & 0.25 & 0.75 & 1.13 \\
\hline & 92 & 0.10 & 1.01 & 2.92 & 0.90 & 1.74 & 0.29 & 0.71 & 1.10 \\
\hline & 129 & 0.14 & 1.74 & 3.34 & 1.22 & 1.76 & 0.31 & 0.69 & 1.16 \\
\hline & 171 & 0.19 & 1.71 & 2.57 & 1.77 & 1.87 & 0.37 & 0.63 & 1.27 \\
\hline \multirow[t]{5}{*}{0.76} & 61 & 0.04 & 1.99 & 7.56 & 0.19 & 1.46 & 0.10 & 0.90 & 1.06 \\
\hline & 77 & 0.05 & 1.30 & 3.32 & 0.36 & 1.86 & 0.16 & 0.84 & 1.12 \\
\hline & 103 & 0.07 & 1.27 & 3.56 & 0.50 & 1.65 & 0.19 & 0.81 & 1.04 \\
\hline & 122 & 0.08 & 1.63 & 5.07 & 0.53 & 1.42 & 0.21 & 0.79 & 1.18 \\
\hline & 131 & 0.09 & 1.62 & 3.39 & 0.67 & 1.92 & 0.25 & 0.75 & 0.95 \\
\hline
\end{tabular}




\begin{tabular}{|c|c|c|c|c|c|c|c|c|c|}
\hline & 152 & 0.10 & 1.60 & 3.22 & 0.83 & 1.81 & 0.27 & 0.73 & 1.08 \\
\hline & 220 & 0.15 & 1.91 & 2.95 & 1.41 & 1.72 & 0.35 & 0.65 & 1.00 \\
\hline & 280 & 0.19 & 1.97 & 3.87 & 2.02 & 1.06 & 0.49 & 0.51 & 1.03 \\
\hline \multirow[t]{8}{*}{1.06} & 82 & 0.04 & 1.29 & 3.77 & 0.22 & 1.85 & 0.10 & 0.90 & 0.99 \\
\hline & 102 & 0.05 & 0.84 & 3.14 & 0.35 & 1.84 & 0.15 & 0.85 & 1.00 \\
\hline & 141 & 0.07 & 0.61 & 2.76 & 0.48 & 1.87 & 0.18 & 0.82 & 1.06 \\
\hline & 174 & 0.08 & 1.19 & 3.37 & 0.66 & 1.69 & 0.23 & 0.77 & 1.08 \\
\hline & 191 & 0.09 & 1.28 & 3.21 & 0.74 & 1.70 & 0.26 & 0.74 & 1.18 \\
\hline & 210 & 0.10 & 1.03 & 2.48 & 0.96 & 1.88 & 0.28 & 0.72 & 1.14 \\
\hline & 301 & 0.14 & 1.52 & 2.57 & 1.58 & 1.70 & 0.35 & 0.65 & 1.15 \\
\hline & 389 & 0.18 & 2.14 & 2.77 & 2.25 & 1.62 & 0.38 & 0.62 & 1.12 \\
\hline \multirow[t]{8}{*}{1.36} & 109 & 0.04 & 0.52 & 2.83 & 0.27 & 1.81 & 0.12 & 0.88 & 1.02 \\
\hline & 150 & 0.05 & 0.64 & 3.10 & 0.40 & 1.72 & 0.16 & 0.84 & 1.02 \\
\hline & 178 & 0.07 & 0.70 & 2.62 & 0.54 & 1.72 & 0.21 & 0.79 & 0.94 \\
\hline & 221 & 0.08 & 0.83 & 2.54 & 0.75 & 1.77 & 0.24 & 0.76 & 1.13 \\
\hline & 238 & 0.09 & 0.84 & 2.76 & 0.81 & 1.79 & 0.24 & 0.76 & 1.23 \\
\hline & 272 & 0.10 & 1.13 & 2.94 & 0.91 & 1.74 & 0.28 & 0.72 & 1.13 \\
\hline & 383 & 0.14 & 2.11 & 2.87 & 1.64 & 1.69 & 0.35 & 0.65 & 1.00 \\
\hline & 504 & 0.18 & 2.79 & 3.06 & 2.35 & 1.55 & 0.37 & 0.63 & 1.26 \\
\hline
\end{tabular}

Table 5c: FBM parameters retrieved by using Equation $\mathrm{S} 2$ to fit the fluorescence decays of DNA-EB quenched by $\mathrm{Ni}^{2+}$ cations with an $\mathrm{Na}_{2} \mathrm{SO}_{4}$ concentration of $1.25 \times 10^{-2} \mathrm{M}$.

\begin{tabular}{|c|c|c|c|c|c|c|c|c|c|}
\hline $\begin{array}{c}{[\mathrm{DNA}]} \\
\mathrm{mM} \text { of } \mathrm{bp}\end{array}$ & {$\left[\mathrm{Ni}^{2+}\right](\mu \mathrm{M})$} & {$\left[\mathrm{Ni}^{2+}\right] /[\mathrm{P}]$} & $\begin{array}{l}k_{\mathrm{e}}[\text { blob] } \\
\times 10^{7} \mathrm{~s}^{-1} \\
\end{array}$ & $\begin{aligned} & k_{\text {blob }} \\
& \times 10^{7} \mathrm{~s}^{-1} \\
&\end{aligned}$ & $<\mathrm{n}>$ & $\tau_{\text {fast }}$ & $a_{\text {fast }}$ & $a_{2}$ & $\chi^{2}$ \\
\hline \multirow[t]{4}{*}{0.30} & 13 & 0.02 & 2.07 & 8.75 & 0.07 & 0.19 & 0.19 & 0.81 & 1.10 \\
\hline & 21 & 0.03 & 0.56 & 2.83 & 0.18 & 1.73 & 0.07 & 0.93 & 1.22 \\
\hline & 24 & 0.04 & 0.81 & 26.26 & 0.21 & 1.83 & 0.08 & 0.92 & 1.08 \\
\hline & 33 & 0.05 & 0.21 & 2.00 & 0.33 & 1.96 & 0.10 & 0.90 & 1.21 \\
\hline
\end{tabular}




\begin{tabular}{|c|c|c|c|c|c|c|c|c|c|}
\hline & 40 & 0.07 & 0.00 & 1.52 & 0.46 & 2.08 & 0.13 & 0.87 & 1.12 \\
\hline & 53 & 0.09 & 0.59 & 2.27 & 0.47 & 1.83 & 0.16 & 0.84 & 1.05 \\
\hline & 61 & 0.10 & 0.47 & 1.88 & 0.57 & 2.09 & 0.18 & 0.82 & 1.16 \\
\hline & 85 & 0.14 & 0.84 & 2.60 & 0.69 & 2.00 & 0.22 & 0.78 & 1.04 \\
\hline \multirow[t]{9}{*}{0.45} & 30 & 0.03 & 0.00 & 1.80 & 0.22 & 2.58 & 0.08 & 0.92 & 1.16 \\
\hline & 37 & 0.04 & 0.55 & 2.36 & 0.23 & 2.29 & 0.08 & 0.92 & 1.23 \\
\hline & 50 & 0.05 & 0.77 & 2.99 & 0.28 & 1.77 & 0.12 & 0.88 & 1.03 \\
\hline & 60 & 0.07 & 0.76 & 3.13 & 0.31 & 1.97 & 0.13 & 0.87 & 1.07 \\
\hline & 72 & 0.08 & 1.09 & 3.62 & 0.38 & 1.65 & 0.15 & 0.85 & 1.25 \\
\hline & 80 & 0.09 & 1.13 & 2.86 & 0.44 & 2.05 & 0.16 & 0.84 & 1.18 \\
\hline & 91 & 0.10 & 0.57 & 2.45 & 0.58 & 1.81 & 0.18 & 0.82 & 1.23 \\
\hline & 127 & 0.14 & 1.01 & 2.66 & 0.70 & 1.98 & 0.22 & 0.78 & 1.04 \\
\hline & 167 & 0.18 & 1.12 & 2.68 & 0.90 & 1.84 & 0.25 & 0.75 & 1.13 \\
\hline \multirow[t]{8}{*}{0.76} & 61 & 0.04 & 1.32 & 4.99 & 0.17 & 1.51 & 0.09 & 0.91 & 1.12 \\
\hline & 84 & 0.06 & 1.16 & 4.55 & 0.25 & 1.68 & 0.11 & 0.89 & 1.00 \\
\hline & 100 & 0.07 & 1.29 & 3.95 & 0.31 & 1.74 & 0.13 & 0.87 & 1.08 \\
\hline & 121 & 0.08 & 1.21 & 3.67 & 0.39 & 1.79 & 0.17 & 0.83 & 1.00 \\
\hline & 134 & 0.09 & 0.97 & 2.63 & 0.50 & 1.89 & 0.20 & 0.80 & 1.02 \\
\hline & 150 & 0.10 & 0.75 & 2.61 & 0.59 & 1.78 & 0.21 & 0.79 & 1.02 \\
\hline & 217 & 0.14 & 1.67 & 3.36 & 0.83 & 1.77 & 0.27 & 0.73 & 1.10 \\
\hline & 284 & 0.19 & 1.56 & 2.85 & 1.19 & 1.69 & 0.30 & 0.70 & 1.00 \\
\hline \multirow[t]{9}{*}{1.06} & 70 & 0.03 & 1.55 & 4.13 & 0.14 & 1.86 & 0.08 & 0.92 & 1.16 \\
\hline & 85 & 0.04 & 1.08 & 3.70 & 0.18 & 2.20 & 0.08 & 0.92 & 1.12 \\
\hline & 117 & 0.05 & 1.00 & 3.19 & 0.29 & 1.95 & 0.12 & 0.88 & 1.07 \\
\hline & 139 & 0.07 & 1.26 & 5.33 & 0.29 & 1.51 & 0.14 & 0.86 & 1.13 \\
\hline & 170 & 0.08 & 1.63 & 4.54 & 0.35 & 1.77 & 0.17 & 0.83 & 1.03 \\
\hline & 186 & 0.09 & 1.00 & 3.23 & 0.50 & 1.61 & 0.20 & 0.80 & 1.13 \\
\hline & 210 & 0.10 & 1.24 & 3.60 & 0.52 & 1.84 & 0.20 & 0.80 & 1.08 \\
\hline & 300 & 0.14 & 2.02 & 4.74 & 0.78 & 1.42 & 0.27 & 0.73 & 1.04 \\
\hline & 384 & 0.18 & 1.55 & 2.76 & 1.27 & 1.76 & 0.32 & 0.68 & 1.10 \\
\hline 1.36 & 94 & 0.03 & 0.37 & 2.31 & 0.23 & 1.96 & 0.11 & 0.89 & 1.05 \\
\hline
\end{tabular}






Table 5d: FBM parameters retrieved by using Equation $\mathrm{S} 2$ to fit the fluorescence decays of DNA-EB quenched by $\mathrm{Ni}^{2+}$ cations with an $\mathrm{Na}_{2} \mathrm{SO}_{4}$ concentration of $2.1 \times 10^{-2} \mathrm{M}$.

\begin{tabular}{|c|c|c|c|c|c|c|c|c|c|}
\hline $\begin{array}{c}{[\mathrm{DNA}]} \\
\mathrm{mM} \text { of bp }\end{array}$ & {$\left[\mathrm{Ni}^{2+}\right](\mu \mathrm{M})$} & {$\left[\mathrm{Ni}^{2+}\right] /[\mathrm{P}]$} & $\begin{array}{c}k_{\mathrm{e}}[b l o b] \\
\times 10^{7} \mathrm{~s}^{-1} \\
\end{array}$ & $\begin{aligned} & k_{\text {blob }} \\
& \times 10^{7} \mathrm{~s}^{-1} \\
&\end{aligned}$ & $<\mathrm{n}>$ & $\tau_{\text {fast }}$ & $a_{\text {fast }}$ & $a_{2}$ & $\chi^{2}$ \\
\hline \multirow[t]{6}{*}{0.30} & 12 & 0.02 & 0.49 & 1.97 & 0.08 & 2.53 & 0.03 & 0.97 & 1.10 \\
\hline & 20 & 0.03 & 0.50 & 2.51 & 0.13 & 1.70 & 0.06 & 0.94 & 1.03 \\
\hline & 40 & 0.07 & 0.73 & 2.90 & 0.22 & 1.78 & 0.10 & 0.90 & 1.05 \\
\hline & 60 & 0.10 & 0.97 & 3.07 & 0.30 & 1.64 & 0.13 & 0.87 & 1.20 \\
\hline & 85 & 0.14 & 1.20 & 3.45 & 0.36 & 1.95 & 0.14 & 0.86 & 1.05 \\
\hline & 112 & 0.18 & 1.12 & 3.53 & 0.45 & 1.56 & 0.16 & 0.84 & 1.02 \\
\hline \multirow[t]{7}{*}{0.45} & 36 & 0.04 & 0.18 & 1.83 & 0.21 & 2.63 & 0.07 & 0.93 & 1.07 \\
\hline & 50 & 0.06 & 1.06 & 3.37 & 0.20 & 2.19 & 0.09 & 0.91 & 1.09 \\
\hline & 60 & 0.07 & 0.27 & 2.11 & 0.32 & 1.82 & 0.11 & 0.89 & 1.05 \\
\hline & 73 & 0.08 & 0.24 & 1.71 & 0.38 & 2.71 & 0.13 & 0.87 & 1.13 \\
\hline & 80 & 0.09 & 0.62 & 2.38 & 0.39 & 1.97 & 0.13 & 0.87 & 1.01 \\
\hline & 90 & 0.10 & 0.70 & 2.79 & 0.47 & 1.86 & 0.13 & 0.87 & 1.10 \\
\hline & 115 & 0.13 & 1.34 & 3.44 & 0.56 & 1.88 & 0.19 & 0.81 & 0.92 \\
\hline \multirow[t]{6}{*}{0.76} & 30 & 0.02 & 0.27 & 2.11 & 0.08 & 1.61 & 0.04 & 0.96 & 0.99 \\
\hline & 50 & 0.03 & 0.99 & 3.44 & 0.14 & 1.69 & 0.08 & 0.92 & 1.04 \\
\hline & 61 & 0.04 & 0.25 & 1.87 & 0.25 & 1.67 & 0.11 & 0.89 & 1.04 \\
\hline & 83 & 0.05 & 0.99 & 2.63 & 0.26 & 1.95 & 0.12 & 0.88 & 1.10 \\
\hline & 100 & 0.07 & 1.11 & 3.87 & 0.26 & 1.63 & 0.12 & 0.88 & 1.07 \\
\hline & 121 & 0.08 & 0.92 & 2.11 & 0.40 & 2.16 & 0.16 & 0.84 & 1.18 \\
\hline
\end{tabular}




\begin{tabular}{|c|c|c|c|c|c|c|c|c|c|}
\hline & 134 & 0.09 & 1.21 & 3.98 & 0.34 & 1.83 & 0.15 & 0.85 & 1.04 \\
\hline & 150 & 0.10 & 1.12 & 3.41 & 0.39 & 1.98 & 0.17 & 0.83 & 1.17 \\
\hline & 215 & 0.14 & 0.98 & 2.64 & 0.71 & 1.85 & 0.23 & 0.77 & 1.05 \\
\hline \multirow[t]{6}{*}{1.06} & 35 & 0.02 & 0.29 & 2.29 & 0.16 & 2.16 & 0.03 & 0.97 & 1.08 \\
\hline & 70 & 0.03 & 0.08 & 2.13 & 0.20 & 0.91 & 0.11 & 0.89 & 1.07 \\
\hline & 140 & 0.07 & 0.82 & 2.24 & 0.34 & 2.10 & 0.13 & 0.87 & 1.24 \\
\hline & 211 & 0.10 & 1.22 & 3.58 & 0.47 & 1.80 & 0.18 & 0.82 & 1.04 \\
\hline & 348 & 0.16 & 1.72 & 3.82 & 0.70 & 1.46 & 0.24 & 0.76 & 0.99 \\
\hline & 399 & 0.19 & 1.26 & 3.62 & 0.97 & 1.72 & 0.28 & 0.72 & 1.11 \\
\hline \multirow[t]{8}{*}{1.36} & 57 & 0.02 & 0.00 & 2.04 & 0.16 & 1.57 & 0.06 & 0.94 & 1.04 \\
\hline & 99 & 0.04 & 0.30 & 2.46 & 0.24 & 1.49 & 0.10 & 0.90 & 1.09 \\
\hline & 113 & 0.04 & 0.29 & 2.29 & 0.29 & 1.99 & 0.09 & 0.91 & 1.14 \\
\hline & 152 & 0.06 & 0.49 & 2.52 & 0.35 & 1.81 & 0.14 & 0.86 & 1.15 \\
\hline & 246 & 0.09 & 1.26 & 3.62 & 0.45 & 1.68 & 0.19 & 0.81 & 1.06 \\
\hline & 272 & 0.10 & 1.18 & 3.72 & 0.49 & 1.56 & 0.20 & 0.80 & 1.10 \\
\hline & 378 & 0.14 & 0.99 & 2.63 & 0.68 & 1.77 & 0.23 & 0.77 & 1.11 \\
\hline & 506 & 0.19 & 1.11 & 3.87 & 1.00 & 1.79 & 0.28 & 0.72 & 1.14 \\
\hline
\end{tabular}

Table 5e: FBM parameters retrieved by using Equation $\mathrm{S} 2$ to fit the fluorescence decays of DNA-EB quenched by $\mathrm{Ni}^{2+}$ cations with an $\mathrm{Na}_{2} \mathrm{SO}_{4}$ concentration of $3 \times 10^{-2} \mathrm{M}$.

\begin{tabular}{|c|c|c|c|c|c|c|c|c|c|}
\hline $\begin{array}{c}{[\mathrm{DNA}]} \\
\mathrm{mM} \text { of } \mathrm{bp}\end{array}$ & {$\left[\mathrm{Ni}^{2+}\right](\mu \mathrm{M})$} & {$\left[\mathrm{Ni}^{2+}\right] /[\mathrm{P}]$} & $\begin{array}{l}k_{\mathrm{e}}[b l o b] \\
\times 10^{7} \mathrm{~s}^{-1} \\
\end{array}$ & $\begin{aligned} & k_{\text {blob }} \\
& \times 10^{7} \mathrm{~s}^{-1} \\
&\end{aligned}$ & $<\mathrm{n}>$ & $\tau_{\text {fast }}$ & $a_{f a s t}$ & $a_{2}$ & $\chi^{2}$ \\
\hline \multirow[t]{7}{*}{0.30} & 13 & 0.02 & 0.08 & 2.13 & 0.09 & 0.61 & 0.04 & 0.96 & 1.15 \\
\hline & 20 & 0.03 & 0.00 & 2.14 & 0.14 & 0.93 & 0.05 & 0.95 & 1.09 \\
\hline & 24 & 0.04 & 0.30 & 2.92 & 0.14 & 0.45 & 0.12 & 0.88 & 1.13 \\
\hline & 41 & 0.07 & 0.00 & 1.45 & 0.23 & 2.36 & 0.08 & 0.92 & 1.08 \\
\hline & 60 & 0.10 & 0.27 & 2.38 & 0.31 & 1.52 & 0.09 & 0.91 & 0.98 \\
\hline & 76 & 0.13 & 0.71 & 1.22 & 0.36 & 1.82 & 0.10 & 0.90 & 1.03 \\
\hline & 112 & 0.18 & 0.73 & 1.11 & 0.53 & 1.55 & 0.15 & 0.85 & 1.09 \\
\hline
\end{tabular}




\begin{tabular}{|c|c|c|c|c|c|c|c|c|c|}
\hline \multirow[t]{8}{*}{0.45} & 30 & 0.03 & 1.79 & 2.75 & 0.12 & 2.17 & 0.05 & 0.95 & 0.98 \\
\hline & 37 & 0.04 & 1.45 & 3.20 & 0.14 & 1.49 & 0.06 & 0.94 & 1.10 \\
\hline & 50 & 0.06 & 0.95 & 2.94 & 0.21 & 2.53 & 0.08 & 0.92 & 1.00 \\
\hline & 60 & 0.07 & 1.64 & 3.70 & 0.25 & 1.85 & 0.10 & 0.90 & 1.00 \\
\hline & 73 & 0.08 & 1.31 & 3.25 & 0.29 & 1.93 & 0.11 & 0.89 & 1.05 \\
\hline & 80 & 0.09 & 0.79 & 2.05 & 0.28 & 2.47 & 0.09 & 0.91 & 1.01 \\
\hline & 90 & 0.10 & 0.34 & 1.24 & 0.32 & 3.07 & 0.09 & 0.91 & 0.96 \\
\hline & 127 & 0.14 & 0.14 & 2.25 & 0.45 & 1.97 & 0.14 & 0.86 & 1.15 \\
\hline \multirow[t]{8}{*}{0.76} & 31 & 0.02 & 1.14 & 4.44 & 0.07 & 1.81 & 0.03 & 0.97 & 1.00 \\
\hline & 50 & 0.03 & 1.33 & 5.44 & 0.11 & 0.96 & 0.08 & 0.92 & 1.02 \\
\hline & 61 & 0.04 & 0.63 & 3.00 & 0.16 & 2.14 & 0.06 & 0.94 & 1.07 \\
\hline & 83 & 0.06 & 0.68 & 3.27 & 0.22 & 1.78 & 0.09 & 0.91 & 1.09 \\
\hline & 100 & 0.07 & 0.79 & 3.04 & 0.25 & 1.87 & 0.11 & 0.89 & 1.03 \\
\hline & 133 & 0.09 & 0.88 & 3.20 & 0.31 & 2.16 & 0.11 & 0.89 & 0.99 \\
\hline & 217 & 0.14 & 0.56 & 2.57 & 0.49 & 1.83 & 0.18 & 0.82 & 0.89 \\
\hline & 283 & 0.19 & 0.74 & 2.86 & 0.59 & 1.71 & 0.20 & 0.80 & 1.07 \\
\hline \multirow[t]{9}{*}{1.06} & 43 & 0.02 & 1.26 & 12.26 & 0.06 & 0.65 & 0.05 & 0.95 & 1.07 \\
\hline & 70 & 0.03 & 1.30 & 6.86 & 0.13 & 0.95 & 0.09 & 0.91 & 1.07 \\
\hline & 85 & 0.04 & 0.16 & 2.52 & 0.15 & 1.59 & 0.08 & 0.92 & 1.06 \\
\hline & 117 & 0.05 & 1.26 & 5.63 & 0.18 & 1.17 & 0.11 & 0.89 & 1.05 \\
\hline & 141 & 0.07 & 0.94 & 3.78 & 0.24 & 1.53 & 0.13 & 0.87 & 1.05 \\
\hline & 169 & 0.08 & 1.12 & 3.90 & 0.31 & 1.63 & 0.14 & 0.86 & 1.03 \\
\hline & 210 & 0.10 & 0.43 & 2.06 & 0.40 & 1.94 & 0.15 & 0.85 & 1.06 \\
\hline & 299 & 0.14 & 1.00 & 2.35 & 0.54 & 2.04 & 0.20 & 0.80 & 1.14 \\
\hline & 390 & 0.18 & 1.26 & 3.36 & 0.72 & 1.94 & 0.21 & 0.79 & 1.22 \\
\hline \multirow[t]{6}{*}{1.36} & 33 & 0.01 & 0.11 & 2.95 & 0.07 & 0.83 & 0.07 & 0.93 & 0.94 \\
\hline & 94 & 0.03 & 0.38 & 2.64 & 0.19 & 1.49 & 0.09 & 0.91 & 1.01 \\
\hline & 111 & 0.04 & 0.41 & 2.38 & 0.23 & 1.69 & 0.09 & 0.91 & 1.17 \\
\hline & 184 & 0.07 & 0.92 & 2.61 & 0.33 & 2.16 & 0.13 & 0.87 & 1.09 \\
\hline & 219 & 0.08 & 1.01 & 3.01 & 0.37 & 1.76 & 0.15 & 0.85 & 1.23 \\
\hline & 271 & 0.10 & 0.83 & 2.40 & 0.50 & 1.84 & 0.18 & 0.82 & 1.03 \\
\hline
\end{tabular}




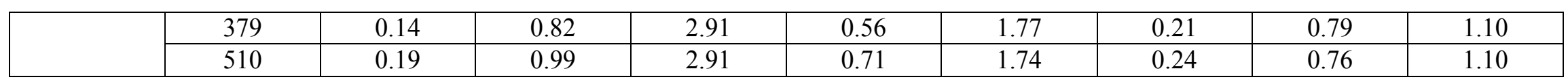

Table 6a: FBM parameters retrieved by using Equation S2 to fit the fluorescence decays of DNA-EB quenched by $\mathrm{Cu}^{2+}$ cations with an $\mathrm{NaCl}$ concentration of $1 \times 10^{-2} \mathrm{M}$.

\begin{tabular}{|c|c|c|c|c|c|c|c|c|c|}
\hline $\begin{array}{c}{[\mathrm{DNA}]} \\
\mathrm{mM} \text { of } \mathrm{bp}\end{array}$ & {$\left[\mathrm{Cu}^{2+}\right](\mu \mathrm{M})$} & {$\left[\mathrm{Cu}^{2+}\right] /[\mathrm{P}]$} & $\begin{array}{l}k_{\mathrm{e}}[b l o b] \\
\times 10^{7} \mathrm{~s}^{-1}\end{array}$ & $\begin{aligned} & k_{\text {blob }} \\
& \times 10^{7} \mathrm{~s}^{-1}\end{aligned}$ & $<\mathrm{n}>$ & $\tau_{\text {fast }}$ & $a_{\text {fast }}$ & $a_{2}$ & $\chi^{2}$ \\
\hline \multirow[t]{11}{*}{0.33} & 11 & 0.02 & 0.64 & 1.89 & 0.17 & 1.87 & 0.05 & 0.95 & 0.95 \\
\hline & 18 & 0.03 & 0.96 & 3.50 & 0.21 & 1.25 & 0.08 & 0.92 & 1.07 \\
\hline & 30 & 0.05 & 1.22 & 6.08 & 0.31 & 0.87 & 0.12 & 0.88 & 1.11 \\
\hline & 42 & 0.07 & 1.15 & 4.55 & 0.49 & 1.60 & 0.13 & 0.87 & 1.08 \\
\hline & 52 & 0.09 & 1.04 & 3.75 & 0.55 & 1.51 & 0.19 & 0.81 & 0.96 \\
\hline & 59 & 0.10 & 1.31 & 4.35 & 0.77 & 1.51 & 0.20 & 0.80 & 1.06 \\
\hline & 67 & 0.11 & 1.53 & 5.31 & 0.84 & 1.18 & 0.23 & 0.77 & 1.13 \\
\hline & 80 & 0.13 & 1.50 & 4.54 & 0.96 & 1.43 & 0.25 & 0.75 & 1.25 \\
\hline & 87 & 0.14 & 1.10 & 3.38 & 1.18 & 1.77 & 0.26 & 0.74 & 1.10 \\
\hline & 97 & 0.16 & 1.08 & 3.39 & 1.36 & 1.64 & 0.29 & 0.71 & 1.12 \\
\hline & 102 & 0.17 & 1.08 & 3.39 & 1.60 & 1.64 & 0.29 & 0.71 & 1.12 \\
\hline \multirow[t]{11}{*}{0.50} & 18 & 0.02 & 0.80 & 5.24 & 0.12 & 1.39 & 0.05 & 0.95 & 1.06 \\
\hline & 26 & 0.03 & 0.98 & 5.85 & 0.19 & 0.80 & 0.08 & 0.92 & 0.92 \\
\hline & 44 & 0.05 & 0.98 & 5.08 & 0.30 & 1.27 & 0.10 & 0.90 & 0.96 \\
\hline & 64 & 0.07 & 1.11 & 4.37 & 0.48 & 1.66 & 0.15 & 0.85 & 1.06 \\
\hline & 82 & 0.09 & 0.62 & 2.82 & 0.77 & 2.07 & 0.19 & 0.81 & 1.09 \\
\hline & 92 & 0.10 & 0.95 & 3.94 & 0.83 & 1.40 & 0.19 & 0.81 & 1.01 \\
\hline & 108 & 0.12 & 1.23 & 4.16 & 0.91 & 1.68 & 0.21 & 0.79 & 1.12 \\
\hline & 118 & 0.13 & 1.33 & 4.73 & 0.99 & 1.42 & 0.21 & 0.79 & 0.99 \\
\hline & 133 & 0.15 & 1.31 & 4.30 & 1.14 & 1.60 & 0.24 & 0.76 & 1.02 \\
\hline & 149 & 0.16 & 1.39 & 4.37 & 1.41 & 1.54 & 0.27 & 0.73 & 1.21 \\
\hline & 159 & 0.17 & 1.51 & 4.65 & 1.44 & 1.28 & 0.27 & 0.73 & 1.16 \\
\hline
\end{tabular}




\begin{tabular}{|c|c|c|c|c|c|c|c|c|c|}
\hline \multirow[t]{11}{*}{0.62} & 23 & 0.02 & 0.78 & 3.86 & 0.13 & 1.07 & 0.13 & 0.87 & 1.07 \\
\hline & 35 & 0.03 & 0.69 & 4.05 & 0.23 & 1.29 & 0.08 & 0.92 & 1.04 \\
\hline & 58 & 0.05 & 0.95 & 5.62 & 0.36 & 0.66 & 0.15 & 0.85 & 0.99 \\
\hline & 80 & 0.07 & 0.93 & 4.02 & 0.54 & 1.62 & 0.14 & 0.86 & 1.06 \\
\hline & 101 & 0.09 & 1.20 & 5.14 & 0.62 & 1.11 & 0.17 & 0.83 & 1.00 \\
\hline & 116 & 0.10 & 1.32 & 4.91 & 0.72 & 1.41 & 0.18 & 0.82 & 1.03 \\
\hline & 132 & 0.12 & 1.50 & 5.30 & 0.85 & 1.41 & 0.19 & 0.81 & 1.12 \\
\hline & 151 & 0.13 & 1.31 & 4.29 & 1.15 & 1.53 & 0.23 & 0.77 & 1.15 \\
\hline & 166 & 0.15 & 1.48 & 4.91 & 1.26 & 1.28 & 0.24 & 0.76 & 1.11 \\
\hline & 183 & 0.16 & 1.19 & 3.53 & 1.69 & 1.62 & 0.29 & 0.71 & 1.07 \\
\hline & 195 & 0.17 & 1.66 & 4.54 & 1.52 & 1.37 & 0.27 & 0.73 & 1.52 \\
\hline \multirow[t]{11}{*}{0.73} & 27 & 0.02 & 2.12 & 6.52 & 0.09 & 2.13 & 0.05 & 0.95 & 1.07 \\
\hline & 42 & 0.03 & 1.20 & 5.34 & 0.17 & 1.56 & 0.07 & 0.93 & 1.00 \\
\hline & 69 & 0.05 & 0.95 & 4.90 & 0.33 & 1.54 & 0.10 & 0.90 & 0.94 \\
\hline & 92 & 0.07 & 1.29 & 6.57 & 0.42 & 1.32 & 0.11 & 0.89 & 1.12 \\
\hline & 124 & 0.09 & 1.23 & 4.93 & 0.63 & 1.51 & 0.17 & 0.83 & 0.98 \\
\hline & 138 & 0.10 & 1.35 & 5.26 & 0.66 & 1.29 & 0.18 & 0.82 & 1.12 \\
\hline & 159 & 0.12 & 1.37 & 5.59 & 0.77 & 1.24 & 0.18 & 0.82 & 0.99 \\
\hline & 176 & 0.13 & 1.51 & 5.08 & 1.16 & 1.11 & 0.24 & 0.74 & 0.86 \\
\hline & 195 & 0.14 & 1.61 & 5.55 & 1.16 & 1.07 & 0.23 & 0.77 & 1.11 \\
\hline & 219 & 0.16 & 1.44 & 4.48 & 1.38 & 1.44 & 0.26 & 0.74 & 1.07 \\
\hline & 230 & 0.17 & 1.51 & 4.75 & 1.39 & 1.27 & 0.26 & 0.74 & 1.39 \\
\hline \multirow[t]{9}{*}{0.98} & 36 & 0.02 & 0.52 & 2.71 & 0.14 & 1.40 & 0.07 & 0.93 & 1.11 \\
\hline & 61 & 0.03 & 0.65 & 4.28 & 0.23 & 1.02 & 0.08 & 0.92 & 1.03 \\
\hline & 94 & 0.05 & 0.99 & 5.22 & 0.32 & 1.16 & 0.10 & 0.90 & 0.98 \\
\hline & 130 & 0.07 & 0.63 & 3.03 & 0.51 & 2.11 & 0.14 & 0.86 & 0.92 \\
\hline & 166 & 0.09 & 1.03 & 3.77 & 0.70 & 2.10 & 0.16 & 0.84 & 0.97 \\
\hline & 181 & 0.10 & 1.07 & 4.41 & 0.71 & 1.51 & 0.17 & 0.83 & 1.06 \\
\hline & 217 & 0.12 & 0.77 & 2.99 & 0.93 & 2.17 & 0.20 & 0.80 & 1.00 \\
\hline & 237 & 0.13 & 1.31 & 4.07 & 1.13 & 1.59 & 0.24 & 0.76 & 1.09 \\
\hline & 264 & 0.15 & 0.93 & 3.11 & 1.29 & 2.01 & 0.24 & 0.76 & 0.93 \\
\hline
\end{tabular}




\begin{tabular}{|c|c|c|c|c|c|c|c|c|c|}
\hline & 310 & 0.17 & 1.61 & 4.04 & 1.77 & 1.41 & 0.30 & 0.70 & 1.15 \\
\hline \multirow[t]{11}{*}{1.11} & 43 & 0.02 & 0.76 & 5.57 & 0.13 & 0.51 & 0.11 & 0.89 & 1.11 \\
\hline & 62 & 0.03 & 0.48 & 3.41 & 0.22 & 2.26 & 0.06 & 0.94 & 1.02 \\
\hline & 103 & 0.05 & 0.41 & 2.74 & 0.44 & 1.97 & 0.12 & 0.88 & 1.09 \\
\hline & 146 & 0.07 & 1.05 & 4.86 & 0.53 & 1.42 & 0.14 & 0.86 & 1.04 \\
\hline & 186 & 0.09 & 0.93 & 3.76 & 0.80 & 1.70 & 0.19 & 0.81 & 0.99 \\
\hline & 204 & 0.10 & 1.06 & 4.07 & 0.79 & 1.64 & 0.19 & 0.81 & 1.05 \\
\hline & 236 & 0.12 & 1.13 & 3.85 & 1.02 & 1.71 & 0.21 & 0.79 & 1.02 \\
\hline & 264 & 0.13 & 1.42 & 4.79 & 1.15 & 1.33 & 0.23 & 0.77 & 1.02 \\
\hline & 293 & 0.14 & 1.46 & 4.01 & 1.49 & 1.61 & 0.27 & 0.73 & 0.98 \\
\hline & 327 & 0.16 & 1.49 & 3.61 & 1.86 & 1.67 & 0.30 & 0.70 & 1.22 \\
\hline & 352 & 0.17 & 1.77 & 4.58 & 1.81 & 1.42 & 0.28 & 0.72 & 1.12 \\
\hline \multirow[t]{11}{*}{1.23} & 46 & 0.02 & 1.52 & 0.09 & 0.16 & 1.34 & 0.05 & 0.95 & 1.00 \\
\hline & 69 & 0.03 & 0.00 & 2.36 & 0.27 & 1.15 & 0.09 & 0.91 & 1.10 \\
\hline & 114 & 0.05 & 0.52 & 3.29 & 0.36 & 1.40 & 0.12 & 0.88 & 1.06 \\
\hline & 162 & 0.07 & 0.75 & 3.99 & 0.51 & 1.50 & 0.13 & 0.87 & 1.06 \\
\hline & 204 & 0.09 & 0.84 & 3.60 & 0.72 & 2.02 & 0.16 & 0.84 & 0.98 \\
\hline & 227 & 0.10 & 1.04 & 3.56 & 0.85 & 1.93 & 0.18 & 0.82 & 0.92 \\
\hline & 263 & 0.12 & 1.19 & 4.04 & 1.01 & 1.61 & 0.23 & 0.77 & 0.98 \\
\hline & 298 & 0.13 & 0.88 & 3.38 & 1.25 & 1.71 & 0.24 & 0.76 & 0.97 \\
\hline & 325 & 0.14 & 1.31 & 4.01 & 1.32 & 1.61 & 0.25 & 0.75 & 1.07 \\
\hline & 363 & 0.16 & 1.44 & 4.08 & 1.53 & 1.50 & 0.26 & 0.74 & 1.10 \\
\hline & 387 & 0.17 & 1.69 & 4.15 & 1.83 & 1.49 & 0.30 & 0.70 & 1.09 \\
\hline
\end{tabular}

Table 6b: FBM parameters retrieved by using Equation S2 to fit the fluorescence decays of DNA-EB quenched by $\mathrm{Cu}^{2+}$ cations with an $\mathrm{NaCl}$ concentration of $2.5 \times 10^{-2} \mathrm{M}$.

\begin{tabular}{|c|c|c|c|c|c|c|c|c|c|}
\hline $\begin{array}{c}{[\mathrm{DNA}]} \\
\mathrm{mM} \text { of } \mathrm{bp}\end{array}$ & {$\left[\mathrm{Cu}^{2+}\right](\mu \mathrm{M})$} & {$\left[\mathrm{Cu}^{2+}\right] /[\mathrm{P}]$} & $\begin{array}{l}k_{\mathrm{e}}[b l o b] \\
\times 10^{7} \mathrm{~s}^{-1}\end{array}$ & $\begin{aligned} & k_{\text {blob }} \\
& \times 10^{7} \mathrm{~s}^{-1}\end{aligned}$ & $<\mathrm{n}>$ & $\tau_{\text {fast }}$ & $a_{\text {fast }}$ & $a_{2}$ & $\chi^{2}$ \\
\hline 0.30 & 7 & 0.01 & 0.16 & 3.20 & 0.07 & 1.76 & 0.04 & 0.96 & 1.18 \\
\hline
\end{tabular}




\begin{tabular}{|c|c|c|c|c|c|c|c|c|c|}
\hline & 12 & 0.02 & 1.21 & 3.48 & 0.10 & 2.14 & 0.05 & 0.95 & 1.06 \\
\hline & 17 & 0.03 & 0.71 & 3.41 & 0.16 & 2.31 & 0.05 & 0.95 & 1.04 \\
\hline & 17 & 0.03 & 0.70 & 3.74 & 0.18 & 1.88 & 0.06 & 0.94 & 1.04 \\
\hline & 24 & 0.04 & 0.95 & 3.67 & 0.28 & 1.84 & 0.08 & 0.92 & 1.04 \\
\hline & 28 & 0.05 & 0.18 & 2.16 & 0.39 & 2.81 & 0.10 & 0.90 & 0.96 \\
\hline & 43 & 0.07 & 0.56 & 2.58 & 0.49 & 2.26 & 0.14 & 0.86 & 1.17 \\
\hline & 42 & 0.07 & 0.74 & 3.53 & 0.41 & 1.94 & 0.11 & 0.89 & 1.09 \\
\hline & 53 & 0.09 & 0.71 & 3.11 & 0.62 & 2.00 & 0.16 & 0.84 & 1.08 \\
\hline & 54 & 0.09 & 0.94 & 4.01 & 0.51 & 1.71 & 0.14 & 0.86 & 1.03 \\
\hline & 60 & 0.10 & 0.96 & 3.61 & 0.61 & 2.13 & 0.15 & 0.85 & 1.13 \\
\hline & 61 & 0.10 & 0.85 & 3.13 & 0.65 & 2.20 & 0.17 & 0.83 & 1.02 \\
\hline & 68 & 0.11 & 1.05 & 3.88 & 0.71 & 1.69 & 0.17 & 0.83 & 1.08 \\
\hline & 69 & 0.11 & 0.94 & 3.68 & 0.72 & 1.85 & 0.18 & 0.82 & 1.06 \\
\hline & 75 & 0.12 & 1.22 & 4.43 & 0.76 & 1.69 & 0.17 & 0.83 & 1.09 \\
\hline & 79 & 0.13 & 1.24 & 4.85 & 0.75 & 1.37 & 0.19 & 0.81 & 1.04 \\
\hline & 85 & 0.14 & 0.88 & 3.20 & 1.00 & 2.01 & 0.21 & 0.79 & 1.13 \\
\hline & 86 & 0.14 & 1.17 & 3.90 & 0.84 & 1.75 & 0.20 & 0.80 & 0.98 \\
\hline & 92 & 0.15 & 1.25 & 4.55 & 0.94 & 1.40 & 0.20 & 0.80 & 1.05 \\
\hline & 96 & 0.16 & 1.44 & 5.22 & 0.87 & 1.32 & 0.20 & 0.80 & 1.11 \\
\hline & 100 & 0.17 & 1.34 & 4.37 & 1.09 & 1.55 & 0.22 & 0.78 & 1.06 \\
\hline & 101 & 0.17 & 1.12 & 3.99 & 1.00 & 1.56 & 0.22 & 0.78 & 1.03 \\
\hline 0.45 & 17 & 0.02 & 0.57 & 2.50 & 0.13 & 1.33 & 0.05 & 0.95 & 1.12 \\
\hline & 27 & 0.03 & 0.49 & 3.87 & 0.20 & 1.46 & 0.07 & 0.93 & 1.13 \\
\hline & 44 & 0.05 & 0.00 & 2.08 & 0.38 & 2.35 & 0.10 & 0.90 & 0.92 \\
\hline & 63 & 0.07 & 0.63 & 3.13 & 0.44 & 2.21 & 0.13 & 0.87 & 1.07 \\
\hline & 70 & 0.08 & 0.55 & 2.44 & 0.40 & 2.77 & 0.13 & 0.87 & 1.11 \\
\hline & 81 & 0.09 & 0.79 & 3.43 & 0.56 & 1.92 & 0.15 & 0.85 & 0.93 \\
\hline & 89 & 0.10 & 0.65 & 3.26 & 0.65 & 1.61 & 0.17 & 0.83 & 1.02 \\
\hline & 95 & 0.10 & 1.03 & 4.05 & 0.63 & 1.62 & 0.16 & 0.84 & 1.03 \\
\hline & 104 & 0.11 & 0.87 & 3.46 & 0.74 & 1.98 & 0.19 & 0.81 & 1.04 \\
\hline & 122 & 0.13 & 0.92 & 3.64 & 0.78 & 1.87 & 0.18 & 0.82 & \\
\hline
\end{tabular}




\begin{tabular}{|c|c|c|c|c|c|c|c|c|c|}
\hline & 131 & 0.14 & 1.09 & 4.02 & 0.87 & 1.58 & 0.21 & 0.79 & 0.91 \\
\hline & 133 & 0.15 & 1.01 & 3.82 & 0.81 & 1.99 & 0.18 & 0.82 & 0.99 \\
\hline & 153 & 0.17 & 1.18 & 4.01 & 1.05 & 1.52 & 0.24 & 0.76 & 1.11 \\
\hline \multirow[t]{18}{*}{0.61} & 23 & 0.02 & 1.77 & 9.84 & 0.06 & 0.37 & 0.06 & 0.94 & 0.99 \\
\hline & 35 & 0.03 & 1.15 & 7.31 & 0.11 & 0.93 & 0.06 & 0.94 & 0.97 \\
\hline & 60 & 0.05 & 1.22 & 8.12 & 0.23 & 0.57 & 0.11 & 0.89 & 1.13 \\
\hline & 84 & 0.07 & 1.18 & 5.19 & 0.28 & 1.44 & 0.11 & 0.89 & 1.00 \\
\hline & 85 & 0.07 & 1.23 & 4.47 & 0.38 & 2.08 & 0.12 & 0.88 & 1.14 \\
\hline & 107 & 0.09 & 1.31 & 5.15 & 0.47 & 1.71 & 0.12 & 0.82 & 1.08 \\
\hline & 112 & 0.09 & 1.32 & 6.36 & 0.36 & 0.86 & 0.14 & 0.86 & 1.09 \\
\hline & 122 & 0.10 & 1.36 & 5.85 & 0.50 & 1.19 & 0.13 & 0.87 & 1.06 \\
\hline & 123 & 0.10 & 1.11 & 5.14 & 0.42 & 1.40 & 0.13 & 0.87 & 0.99 \\
\hline & 145 & 0.12 & 1.20 & 4.43 & 0.52 & 2.06 & 0.13 & 0.87 & 1.11 \\
\hline & 158 & 0.13 & 1.31 & 5.10 & 0.80 & 1.29 & 0.20 & 0.80 & 1.18 \\
\hline & 162 & 0.13 & 1.14 & 4.14 & 0.70 & 1.74 & 0.19 & 0.81 & 0.98 \\
\hline & 175 & 0.14 & 1.19 & 4.41 & 0.87 & 1.47 & 0.22 & 0.78 & 1.01 \\
\hline & 178 & 0.15 & 1.68 & 5.91 & 0.77 & 1.61 & 0.17 & 0.83 & 1.10 \\
\hline & 194 & 0.15 & 1.19 & 4.10 & 1.00 & 1.77 & 0.23 & 0.77 & 1.11 \\
\hline & 196 & 0.16 & 1.53 & 5.02 & 1.06 & 1.34 & 0.24 & 0.76 & 1.01 \\
\hline & 207 & 0.17 & 1.56 & 4.98 & 1.11 & 1.24 & 0.25 & 0.75 & 1.10 \\
\hline & 210 & 0.17 & 1.75 & 5.82 & 1.12 & 1.03 & 0.24 & 0.76 & 1.07 \\
\hline \multirow[t]{9}{*}{0.76} & 108 & 0.07 & 1.29 & 6.57 & 0.46 & 1.32 & 0.11 & 0.89 & \\
\hline & 137 & 0.09 & 0.58 & 3.06 & 0.63 & 1.88 & 0.16 & 0.84 & 1.14 \\
\hline & 151 & 0.10 & 0.87 & 4.22 & 0.62 & 1.32 & 0.16 & 0.84 & 1.00 \\
\hline & 175 & 0.12 & 1.04 & 4.37 & 0.74 & 1.41 & 0.19 & 0.81 & 1.14 \\
\hline & 198 & 0.13 & 0.78 & 3.23 & 1.01 & 1.93 & 0.21 & 0.79 & 1.12 \\
\hline & 219 & 0.14 & 0.73 & 2.84 & 1.18 & 2.04 & 0.23 & 0.77 & 1.02 \\
\hline & 247 & 0.16 & 1.15 & 3.38 & 1.34 & 1.79 & 0.27 & 0.73 & 1.07 \\
\hline & 254 & 0.17 & 1.31 & 4.05 & 1.27 & 1.61 & 0.24 & 0.76 & 1.18 \\
\hline & 280 & 0.18 & 1.52 & 4.25 & 1.45 & 1.45 & 0.26 & 0.74 & 1.07 \\
\hline 0.91 & 24 & 0.01 & 0.00 & 1.94 & 0.12 & 1.93 & 0.04 & 0.96 & 1.03 \\
\hline
\end{tabular}




\begin{tabular}{|c|c|c|c|c|c|c|c|c|c|}
\hline & 53 & 0.03 & 0.08 & 3.22 & 0.17 & 0.93 & 0.08 & 0.92 & 1.16 \\
\hline & 76 & 0.04 & 0.37 & 3.96 & 0.26 & 1.03 & 0.09 & 0.91 & 1.04 \\
\hline & 104 & 0.06 & 0.50 & 3.85 & 0.36 & 1.41 & 0.09 & 0.91 & 1.18 \\
\hline & 130 & 0.07 & 0.31 & 2.94 & 0.54 & 1.80 & 0.13 & 0.87 & 1.07 \\
\hline & 153 & 0.08 & 0.52 & 3.16 & 0.58 & 2.00 & 0.15 & 0.85 & 0.98 \\
\hline & 180 & 0.10 & 0.61 & 3.04 & 0.74 & 2.36 & 0.17 & 0.83 & 1.08 \\
\hline & 204 & 0.11 & 0.97 & 3.58 & 0.82 & 1.98 & 0.18 & 0.82 & 1.05 \\
\hline & 233 & 0.13 & 1.05 & 3.89 & 0.96 & 1.56 & 0.21 & 0.79 & 1.12 \\
\hline & 258 & 0.14 & 1.30 & 4.56 & 1.01 & 1.45 & 0.21 & 0.79 & 1.06 \\
\hline & 284 & 0.16 & 1.20 & 3.83 & 1.26 & 1.59 & 0.24 & 0.76 & 1.11 \\
\hline & 308 & 0.17 & 1.33 & 4.08 & 1.44 & 1.39 & 0.26 & 0.74 & 1.18 \\
\hline 1.06 & 42 & 0.02 & 0.08 & 3.31 & 0.14 & 0.39 & 0.12 & 0.88 & 1.04 \\
\hline & 64 & 0.03 & 0.32 & 2.73 & 0.21 & 2.02 & 0.07 & 0.93 & 1.11 \\
\hline & 109 & 0.05 & 0.70 & 3.82 & 0.30 & 1.30 & 0.10 & 0.90 & 1.09 \\
\hline & 149 & 0.07 & 0.63 & 3.27 & 0.43 & 2.07 & 0.11 & 0.89 & 1.01 \\
\hline & 192 & 0.09 & 0.87 & 3.64 & 0.59 & 1.61 & 0.17 & 0.83 & 1.17 \\
\hline & 214 & 0.10 & 0.96 & 3.63 & 0.66 & 1.71 & 0.17 & 0.83 & 1.17 \\
\hline & 245 & 0.12 & 0.99 & 3.99 & 0.80 & 1.65 & 0.18 & 0.82 & 1.08 \\
\hline & 274 & 0.13 & 0.96 & 3.99 & 0.91 & 1.33 & 0.21 & 0.79 & 1.17 \\
\hline & 305 & 0.14 & 1.16 & 3.65 & 1.08 & 1.76 & 0.23 & 0.77 & 1.00 \\
\hline & 338 & 0.16 & 1.42 & 4.29 & 1.22 & 1.42 & 0.24 & 0.76 & 1.04 \\
\hline & 358 & 0.17 & 1.24 & 3.56 & 1.52 & 1.58 & 0.26 & 0.74 & 1.04 \\
\hline
\end{tabular}

Table 6c: FBM parameters retrieved by using Equation S2 to fit the fluorescence decays of DNA-EB quenched by $\mathrm{Cu}^{2+}$ cations with an $\mathrm{NaCl}$ concentration of $4.1 \times 10^{-2} \mathrm{M}$.

\begin{tabular}{|c|c|c|c|c|c|c|c|c|c|}
\hline $\begin{array}{c}{[\mathrm{DNA}]} \\
\mathrm{mM} \text { of } \mathrm{bp}\end{array}$ & {$\left[\mathrm{Cu}^{2+}\right](\mu \mathrm{M})$} & {$\left[\mathrm{Cu}^{2+}\right] /[\mathrm{P}]$} & $\begin{array}{c}k_{\mathrm{e}}[\text { blob] } \\
\times 10^{7} \mathrm{~s}^{-1}\end{array}$ & $\begin{array}{c}k_{\text {blob }} \\
\times 10^{7} \mathrm{~s}^{-1}\end{array}$ & $<\mathrm{n}>$ & $\tau_{\text {fast }}$ & $a_{\text {fast }}$ & $a_{2}$ & $\chi^{2}$ \\
\hline \multirow{2}{*}{0.30} & 8 & 0.01 & 0.00 & 1.98 & 0.09 & 0.98 & 0.02 & 0.98 & 1.00 \\
\cline { 2 - 36 } & 9 & 0.01 & 0.86 & 5.20 & 0.09 & 0.49 & 0.09 & 0.10 \\
\hline
\end{tabular}




\begin{tabular}{|c|c|c|c|c|c|c|c|c|c|}
\hline & 17 & 0.03 & 0.70 & 3.74 & 0.12 & 1.85 & 0.06 & 0.94 & 1.20 \\
\hline & 17 & 0.03 & 0.55 & 3.01 & 0.14 & 1.72 & 0.07 & 0.93 & 1.00 \\
\hline & 25 & 0.04 & 0.39 & 3.24 & 0.21 & 1.06 & 0.08 & 0.92 & 1.14 \\
\hline & 25 & 0.04 & 0.73 & 4.38 & 0.21 & 1.11 & 0.08 & 0.92 & 1.06 \\
\hline & 34 & 0.06 & 0.26 & 2.94 & 0.29 & 1.59 & 0.09 & 0.91 & 1.01 \\
\hline & 34 & 0.06 & 0.53 & 3.19 & 0.28 & 2.01 & 0.08 & 0.92 & 1.10 \\
\hline & 41 & 0.07 & 0.57 & 3.46 & 0.33 & 1.86 & 0.08 & 0.92 & 1.00 \\
\hline & 42 & 0.07 & 0.67 & 3.25 & 0.39 & 1.60 & 0.13 & 0.87 & 1.09 \\
\hline & 52 & 0.09 & 0.82 & 4.33 & 0.43 & 1.90 & 0.11 & 0.89 & 1.12 \\
\hline & 51 & 0.08 & 0.74 & 3.91 & 0.35 & 1.71 & 0.11 & 0.89 & 1.03 \\
\hline & 61 & 0.10 & 0.65 & 3.21 & 0.46 & 2.05 & 0.12 & 0.88 & 1.08 \\
\hline & 59 & 0.10 & 1.11 & 5.22 & 0.36 & 0.93 & 0.14 & 0.86 & 1.06 \\
\hline & 70 & 0.12 & 0.66 & 3.33 & 0.49 & 1.93 & 0.13 & 0.87 & 1.02 \\
\hline & 69 & 0.11 & 1.20 & 4.87 & 0.43 & 0.87 & 0.13 & 0.87 & 1.06 \\
\hline & 76 & 0.13 & 0.91 & 4.29 & 0.52 & 1.46 & 0.15 & 0.85 & 1.02 \\
\hline & 77 & 0.13 & 0.40 & 2.24 & 0.68 & 2.78 & 0.17 & 0.83 & 0.98 \\
\hline & 87 & 0.14 & 1.04 & 4.28 & 0.58 & 1.60 & 0.17 & 0.83 & 0.98 \\
\hline & 86 & 0.14 & 0.75 & 3.06 & 0.77 & 2.17 & 0.18 & 0.82 & 1.04 \\
\hline & 95 & 0.16 & 0.74 & 3.57 & 0.74 & 1.62 & 0.17 & 0.83 & 0.96 \\
\hline & 94 & 0.15 & 1.23 & 4.86 & 0.64 & 1.69 & 0.15 & 0.85 & 0.97 \\
\hline & 104 & 0.17 & 1.06 & 3.99 & 0.70 & 1.76 & 0.17 & 0.83 & 0.96 \\
\hline & 104 & 0.17 & 1.17 & 4.52 & 0.71 & 1.49 & 0.18 & 0.82 & 1.03 \\
\hline 0.45 & 18 & 0.02 & 0.97 & 3.71 & 0.09 & 1.09 & 0.06 & 0.94 & 1.14 \\
\hline & 26 & 0.03 & 0.65 & 3.54 & 0.15 & 1.42 & 0.05 & 0.95 & 1.07 \\
\hline & 46 & 0.05 & 0.42 & 2.72 & 0.31 & 1.85 & 0.09 & 0.91 & 1.10 \\
\hline & 63 & 0.07 & 0.73 & 3.63 & 0.37 & 1.20 & 0.12 & 0.88 & 1.14 \\
\hline & 81 & 0.09 & 0.86 & 3.19 & 0.51 & 1.94 & 0.14 & 0.86 & 1.07 \\
\hline & 85 & 0.09 & 0.87 & 4.24 & 0.46 & 1.39 & 0.12 & 0.88 & 1.19 \\
\hline & 92 & 0.10 & 0.75 & 3.26 & 0.57 & 1.90 & 0.14 & 0.86 & 1.03 \\
\hline & 96 & 0.11 & 0.77 & 3.01 & 0.61 & 2.00 & 0.15 & 0.85 & 1.05 \\
\hline & 104 & 0.11 & 1.02 & 3.54 & 0.66 & 1.80 & 0.17 & 0.83 & 1.12 \\
\hline
\end{tabular}




\begin{tabular}{|c|c|c|c|c|c|c|c|c|c|}
\hline & 119 & 0.13 & 0.89 & 3.38 & 0.81 & 1.87 & 0.18 & 0.82 & 1.11 \\
\hline & 126 & 0.14 & 0.94 & 3.15 & 0.75 & 2.16 & 0.18 & 0.82 & 1.04 \\
\hline & 134 & 0.15 & 1.19 & 3.88 & 0.80 & 1.54 & 0.20 & 0.80 & 1.05 \\
\hline & 144 & 0.16 & 1.05 & 3.68 & 0.87 & 1.83 & 0.20 & 0.80 & 1.00 \\
\hline & 154 & 0.17 & 1.12 & 3.46 & 1.00 & 1.87 & 0.22 & 0.78 & 1.12 \\
\hline \multirow[t]{12}{*}{0.61} & 17 & 0.01 & 0.00 & 2.52 & 0.08 & 0.64 & 0.06 & 0.94 & 1.00 \\
\hline & 33 & 0.03 & 0.00 & 1.86 & 0.14 & 3.38 & 0.03 & 0.97 & 1.10 \\
\hline & 53 & 0.04 & 0.30 & 3.01 & 0.23 & 0.05 & 1.93 & 0.95 & 1.12 \\
\hline & 69 & 0.06 & 0.36 & 2.75 & 0.26 & 2.17 & 0.09 & 0.91 & 1.15 \\
\hline & 86 & 0.07 & 0.81 & 4.00 & 0.31 & 1.79 & 0.10 & 0.90 & 1.06 \\
\hline & 103 & 0.08 & 0.52 & 2.87 & 0.43 & 2.02 & 0.12 & 0.88 & 1.05 \\
\hline & 120 & 0.10 & 0.44 & 2.37 & 0.58 & 2.83 & 0.15 & 0.85 & 1.04 \\
\hline & 136 & 0.11 & 0.97 & 4.45 & 0.50 & 1.26 & 0.14 & 0.86 & 1.08 \\
\hline & 155 & 0.13 & 0.90 & 3.45 & 0.65 & 2.03 & 0.16 & 0.84 & 1.11 \\
\hline & 172 & 0.14 & 0.60 & 2.57 & 0.83 & 2.10 & 0.20 & 0.80 & 1.00 \\
\hline & 188 & 0.16 & 0.68 & 2.93 & 0.93 & 1.94 & 0.20 & 0.80 & 1.04 \\
\hline & 204 & 0.17 & 1.04 & 3.81 & 0.95 & 1.59 & 0.20 & 0.80 & 1.07 \\
\hline \multirow[t]{14}{*}{0.76} & 29 & 0.02 & 0.78 & 5.54 & 0.09 & 0.34 & 0.11 & 0.89 & 1.01 \\
\hline & 45 & 0.03 & 0.14 & 3.19 & 0.15 & 1.45 & 0.05 & 0.95 & 1.12 \\
\hline & 75 & 0.05 & 0.70 & 4.14 & 0.25 & 1.51 & 0.07 & 0.93 & 0.99 \\
\hline & 106 & 0.07 & 0.68 & 3.68 & 0.32 & 1.73 & 0.09 & 0.91 & 1.01 \\
\hline & 119 & 0.08 & 0.77 & 3.89 & 0.43 & 1.81 & 0.11 & 0.89 & 1.12 \\
\hline & 137 & 0.09 & 0.79 & 3.72 & 0.42 & 1.79 & 0.13 & 0.87 & 1.04 \\
\hline & 154 & 0.10 & 0.83 & 3.83 & 0.57 & 1.51 & 0.16 & 0.84 & 0.98 \\
\hline & 164 & 0.11 & 0.66 & 3.01 & 0.69 & 2.12 & 0.16 & 0.84 & 1.02 \\
\hline & 173 & 0.11 & 0.89 & 3.46 & 0.66 & 2.06 & 0.16 & 0.84 & 1.04 \\
\hline & 197 & 0.13 & 0.82 & 3.51 & 0.84 & 1.87 & 0.18 & 0.82 & 1.04 \\
\hline & 210 & 0.14 & 0.13 & 4.91 & 0.76 & 1.33 & 0.18 & 0.82 & 1.03 \\
\hline & 221 & 0.15 & 0.98 & 3.65 & 0.88 & 1.77 & 0.20 & 0.80 & 0.97 \\
\hline & 248 & 0.16 & 1.23 & 4.13 & 1.05 & 1.56 & 0.20 & 0.80 & 1.02 \\
\hline & 257 & 0.17 & 1.16 & 4.32 & 0.95 & 1.36 & 0.22 & 0.78 & 1.09 \\
\hline
\end{tabular}




\begin{tabular}{|c|c|c|c|c|c|c|c|c|c|}
\hline \multirow[t]{12}{*}{0.91} & 25 & 0.01 & 0.00 & 2.74 & 0.08 & 0.48 & 0.07 & 0.93 & 1.06 \\
\hline & 51 & 0.03 & 0.35 & 3.33 & 0.14 & 2.22 & 0.05 & 0.95 & 1.06 \\
\hline & 76 & 0.04 & 0.78 & 4.26 & 0.20 & 1.56 & 0.06 & 0.94 & 1.05 \\
\hline & 102 & 0.06 & 0.60 & 3.68 & 0.30 & 1.25 & 0.11 & 0.89 & 1.10 \\
\hline & 128 & 0.07 & 0.56 & 3.35 & 0.41 & 1.65 & 0.12 & 0.88 & 1.00 \\
\hline & 156 & 0.09 & 0.64 & 3.54 & 0.51 & 1.65 & 0.13 & 0.87 & 1.00 \\
\hline & 181 & 0.10 & 0.79 & 3.55 & 0.60 & 1.51 & 0.16 & 0.84 & 1.15 \\
\hline & 208 & 0.11 & 1.12 & 4.34 & 0.66 & 1.48 & 0.18 & 0.82 & 1.19 \\
\hline & 232 & 0.13 & 1.13 & 4.08 & 0.78 & 1.72 & 0.18 & 0.82 & 1.10 \\
\hline & 258 & 0.14 & 1.09 & 3.98 & 0.91 & 1.47 & 0.20 & 0.80 & 1.09 \\
\hline & 281 & 0.15 & 1.19 & 3.86 & 1.05 & 1.44 & 0.23 & 0.77 & 1.02 \\
\hline & 309 & 0.17 & 1.37 & 4.37 & 1.07 & 1.39 & 0.22 & 0.78 & 0.95 \\
\hline \multirow[t]{11}{*}{1.06} & 43 & 0.02 & 0.15 & 3.43 & 0.13 & 1.62 & 0.03 & 0.97 & 1.09 \\
\hline & 64 & 0.03 & 0.26 & 3.45 & 0.16 & 1.26 & 0.06 & 0.94 & 1.01 \\
\hline & 105 & 0.05 & 0.43 & 3.43 & 0.22 & 1.95 & 0.07 & 0.93 & 1.05 \\
\hline & 149 & 0.07 & 0.83 & 4.39 & 0.30 & 1.27 & 0.10 & 0.90 & 1.07 \\
\hline & 198 & 0.09 & 0.79 & 4.74 & 0.43 & 1.20 & 0.12 & 0.88 & 1.12 \\
\hline & 211 & 0.10 & 0.71 & 3.86 & 0.51 & 1.73 & 0.13 & 0.87 & 1.02 \\
\hline & 243 & 0.11 & 0.81 & 3.85 & 0.63 & 1.65 & 0.15 & 0.85 & 1.05 \\
\hline & 276 & 0.13 & 0.81 & 3.36 & 0.81 & 1.73 & 0.20 & 0.80 & 1.03 \\
\hline & 309 & 0.15 & 1.16 & 4.56 & 0.79 & 1.30 & 0.20 & 0.80 & 1.14 \\
\hline & 339 & 0.16 & 1.24 & 3.98 & 1.02 & 1.76 & 0.22 & 0.78 & 1.10 \\
\hline & 358 & 0.17 & 1.30 & 4.54 & 1.05 & 1.35 & 0.21 & 0.79 & 0.95 \\
\hline
\end{tabular}

Table 7: Slope and intercept for $<\mathrm{n}>\mathrm{vs}\left[\mathrm{Cu}^{2+}\right]$.

\begin{tabular}{|c|c|c|c|c|}
\hline \multirow{2}{*}[\mathrm{Na}_{2}\mathrm{SO}_{4}]{$(\mathrm{M})$} & wt \% DNA & [DNA] (in base pairs) & slope & intercept \\
\hline \multirow{2}{*}{$5 \times 10^{-4}$} & 0.02 & $3.03 \mathrm{E}-04$ & $29729 \pm 2690$ & $-0.68 \pm 0.29$ \\
\cline { 2 - 5 } & 0.03 & $4.55 \mathrm{E}-04$ & $19001 \pm 892$ & $-0.41 \pm 0.11$ \\
\cline { 2 - 5 } & 0.05 & $7.58 \mathrm{E}-04$ & $10316 \pm 286$ & $-0.35 \pm 0.05$ \\
\hline
\end{tabular}




\begin{tabular}{|c|c|c|c|c|}
\hline & 0.07 & $1.06 \mathrm{E}-03$ & $7936 \pm 364$ & $-0.51 \pm 0.10$ \\
\hline & 0.09 & $1.36 \mathrm{E}-03$ & $6189 \pm 884$ & $-0.53 \pm 0.21$ \\
\hline \multirow[t]{5}{*}{$5 \times 10^{-3}$} & 0.02 & $3.03 \mathrm{E}-04$ & $18272 \pm 486$ & $-0.20 \pm 0.04$ \\
\hline & 0.03 & $4.55 \mathrm{E}-04$ & $14356 \pm 500$ & $-0.30 \pm 0.06$ \\
\hline & 0.05 & $7.58 \mathrm{E}-04$ & $9416 \pm 885$ & $-0.30 \pm 0.17$ \\
\hline & 0.07 & $1.06 \mathrm{E}-03$ & $8224 \pm 695$ & $-0.65 \pm 0.20$ \\
\hline & 0.09 & $1.36 \mathrm{E}-03$ & $6268 \pm 383$ & $-0.69 \pm 0.14$ \\
\hline \multirow[t]{5}{*}{$7.5 \times 10^{-3}$} & 0.02 & $3.03 \mathrm{E}-04$ & $15656 \pm 2636$ & 0 \\
\hline & 0.03 & $4.55 \mathrm{E}-04$ & $10428 \pm 209$ & 0 \\
\hline & 0.05 & $7.58 \mathrm{E}-04$ & $7084 \pm 270$ & $-0.10 \pm 0.04$ \\
\hline & 0.07 & $1.06 \mathrm{E}-03$ & $5932 \pm 272$ & $-0.22 \pm 0.06$ \\
\hline & 0.09 & $1.36 \mathrm{E}-03$ & $4368 \pm 222$ & $-0.16 \pm 0.06$ \\
\hline \multirow[t]{5}{*}{$1.25 \times 10^{-2}$} & 0.02 & $3.03 \mathrm{E}-04$ & $11632 \pm 1019$ & 0 \\
\hline & 0.03 & $4.55 \mathrm{E}-04$ & $9197 \pm 170$ & 0 \\
\hline & 0.05 & $7.58 \mathrm{E}-04$ & $6297 \pm 440$ & $-0.10 \pm 0.08$ \\
\hline & 0.07 & $1.06 \mathrm{E}-03$ & $4541 \pm 126$ & $-0.05 \pm 0.03$ \\
\hline & 0.09 & $1.36 \mathrm{E}-03$ & $3396 \pm 86$ & $-0.07 \pm 0.03$ \\
\hline \multirow[t]{6}{*}{$2.1 \times 10^{-2}$} & 0.02 & $3.03 \mathrm{E}-04$ & $8893 \pm 396$ & 0 \\
\hline & 0.03 & $4.55 \mathrm{E}-04$ & $5251 \pm 166$ & 0 \\
\hline & 0.05 & $7.58 \mathrm{E}-04$ & $4405 \pm 320$ & $-0.08 \pm 0.05$ \\
\hline & 0.07 & $1.06 \mathrm{E}-03$ & $3343 \pm 72$ & $-0.05 \pm 0.02$ \\
\hline & 0.08 & $1.27 \mathrm{E}-03$ & $3239 \pm 184$ & $-0.07 \pm 0.05$ \\
\hline & 0.09 & $1.36 \mathrm{E}-03$ & $2685 \pm 132$ & $-0.09 \pm 0.04$ \\
\hline \multirow[t]{8}{*}{$3 \times 10^{-2}$} & 0.02 & $3.03 \mathrm{E}-04$ & $6119 \pm 286$ & 0 \\
\hline & 0.03 & $4.55 \mathrm{E}-04$ & $4662 \pm 190$ & 0 \\
\hline & 0.04 & $6.06 \mathrm{E}-04$ & $3334 \pm 344$ & 0 \\
\hline & 0.05 & $7.58 \mathrm{E}-04$ & $2569 \pm 49$ & 0 \\
\hline & 0.06 & 9.09E-04 & $2746 \pm 95$ & 0 \\
\hline & 0.07 & $1.06 \mathrm{E}-03$ & $2729 \pm 81$ & 0 \\
\hline & 0.08 & $1.21 \mathrm{E}-03$ & $2253 \pm 89$ & 0 \\
\hline & 0.09 & $1.36 \mathrm{E}-03$ & $1778 \pm 42$ & 0 \\
\hline \multirow[t]{2}{*}{$4 \times 10^{-2}$} & 0.02 & $3.03 \mathrm{E}-04$ & $4461 \pm 341$ & 0 \\
\hline & 0.03 & $4.55 \mathrm{E}-04$ & $3085 \pm 126$ & 0 \\
\hline
\end{tabular}




\begin{tabular}{|c|c|c|c|}
\hline \multirow{4}{*}{} & 0.04 & $6.06 \mathrm{E}-04$ & $2110 \pm 126$ \\
\cline { 2 - 4 } & 0.05 & $7.58 \mathrm{E}-04$ & $2432 \pm 89$ \\
\cline { 2 - 4 } & 0.06 & $9.09 \mathrm{E}-04$ & $2212 \pm 94$ \\
\cline { 2 - 4 } & 0.07 & $1.06 \mathrm{E}-03$ & $1776 \pm 55$ \\
\cline { 2 - 4 } & 0.08 & $1.21 \mathrm{E}-03$ & $1825 \pm 44$ \\
\hline
\end{tabular}

Table 8: Slope and intercept for $<\mathrm{n}>\mathrm{vs}\left[\mathrm{Ni}^{2+}\right]$.

\begin{tabular}{|c|c|c|c|c|}
\hline$\left[\mathrm{Na}_{2} \mathrm{SO}_{4}\right](\mathrm{M})$ & wt $\%$ DNA & [DNA] (in base pairs) & slope & intercept \\
\hline \multirow[t]{5}{*}{$5 \times 10^{-4}$} & 0.02 & $3.03 \mathrm{E}-04$ & $21548 \pm 705$ & $-0.11 \pm 0.04$ \\
\hline & 0.03 & $4.55 \mathrm{E}-04$ & $16796 \pm 1029$ & $-0.25 \pm 0.09$ \\
\hline & 0.05 & $7.58 \mathrm{E}-04$ & $12534 \pm 874$ & $-0.63 \pm 0.15$ \\
\hline & 0.07 & $1.06 \mathrm{E}-03$ & $8308 \pm 627$ & $-0.54 \pm 0.14$ \\
\hline & 0.09 & $1.36 \mathrm{E}-03$ & $6552 \pm 207$ & $-0.63 \pm 0.07$ \\
\hline \multirow[t]{5}{*}{$5 \times 10^{-3}$} & 0.02 & $3.03 \mathrm{E}-04$ & $14316 \pm 3101$ & 0 \\
\hline & 0.03 & $4.55 \mathrm{E}-04$ & $11284 \pm 361$ & $-0.18 \pm 0.03$ \\
\hline & 0.05 & $7.58 \mathrm{E}-04$ & $8189 \pm 403$ & $-0.36 \pm 0.06$ \\
\hline & 0.07 & $1.06 \mathrm{E}-03$ & $6652 \pm 324$ & $-0.42 \pm 0.07$ \\
\hline & 0.09 & $1.36 \mathrm{E}-03$ & $5349 \pm 217$ & $-0.41 \pm 0.06$ \\
\hline \multirow[t]{5}{*}{$1.25 \times 10^{-2}$} & 0.02 & $3.03 \mathrm{E}-04$ & $8934 \pm 394$ & 0 \\
\hline & 0.03 & $4.55 \mathrm{E}-04$ & $5526 \pm 124$ & 0 \\
\hline & 0.05 & $7.58 \mathrm{E}-04$ & $4598 \pm 157$ & $-0.13 \pm 0.03$ \\
\hline & 0.07 & $1.06 \mathrm{E}-03$ & $3428 \pm 268$ & $-0.15 \pm 0.06$ \\
\hline & 0.09 & $1.36 \mathrm{E}-03$ & $3039 \pm 104$ & $-0.10 \pm 0.03$ \\
\hline \multirow[t]{5}{*}{$2.1 \times 10^{-2}$} & 0.02 & $3.03 \mathrm{E}-04$ & $4367 \pm 245$ & 0 \\
\hline & 0.03 & $4.55 \mathrm{E}-04$ & $3802 \pm 273$ & 0 \\
\hline & 0.05 & $7.58 \mathrm{E}-04$ & $3018 \pm 139$ & 0 \\
\hline & 0.07 & $1.06 \mathrm{E}-03$ & $2274 \pm 107$ & 0 \\
\hline & 0.09 & $1.36 \mathrm{E}-03$ & $1925 \pm 67$ & 0 \\
\hline $3 \times 10^{-2}$ & 0.02 & $3.03 \mathrm{E}-04$ & $4944 \pm 182$ & 0 \\
\hline
\end{tabular}




\begin{tabular}{|c|c|c|c|c|}
\hline \multirow{4}{*}{} & 0.03 & $4.55 \mathrm{E}-04$ & $3675 \pm 95$ \\
\cline { 2 - 4 } & 0.05 & $7.58 \mathrm{E}-04$ & $2223 \pm 63$ & 0 \\
\cline { 2 - 4 } & 0.07 & $1.06 \mathrm{E}-03$ & $1821 \pm 26$ \\
\cline { 2 - 4 } & 0.09 & $1.36 \mathrm{E}-03$ & $1545 \pm 74$ \\
\hline
\end{tabular}

Table 9: Slope and intercept for $<\mathrm{n}>$ vs $\left[\mathrm{Cu}^{2+}\right]$.

\begin{tabular}{|c|c|c|c|c|}
\hline$[\mathrm{NaCl}](\mathrm{M})$ & wt $\%$ DNA & [DNA] (in base pairs) & slope & intercept \\
\hline \multirow[t]{7}{*}{$1 \times 10^{-2}$} & 0.022 & $3.28 \mathrm{E}-04$ & $15612 \pm 1255$ & $-0.20 \pm 0.09$ \\
\hline & 0.033 & $4.93 \mathrm{E}-04$ & $9719 \pm 666$ & $-0.10 \pm 0.08$ \\
\hline & 0.041 & $6.18 \mathrm{E}-04$ & $9106 \pm 1099$ & $-0.28 \pm 0.10$ \\
\hline & 0.048 & 7.33E-04 & $7633 \pm 657$ & $-0.33 \pm 0.11$ \\
\hline & 0.065 & $9.83 \mathrm{E}-04$ & $6880 \pm 568$ & $-0.47 \pm 0.13$ \\
\hline & 0.073 & $1.11 \mathrm{E}-03$ & $6779 \pm 537$ & $-0.52 \pm 0.13$ \\
\hline & 0.081 & $1.23 \mathrm{E}-03$ & $5503 \pm 283$ & $-0.41 \pm 0.08$ \\
\hline \multirow[t]{6}{*}{$2.5 \times 10^{-2}$} & 0.02 & $3.01 \mathrm{E}-04$ & $10030 \pm 148$ & 0 \\
\hline & 0.03 & $4.51 \mathrm{E}-04$ & $6666 \pm 418$ & 0 \\
\hline & 0.04 & $6.08 \mathrm{E}-04$ & $6589 \pm 463$ & $-0.30 \pm 0.07$ \\
\hline & 0.05 & $7.58 \mathrm{E}-04$ & $6132 \pm 401$ & $-0.24 \pm 0.08$ \\
\hline & 0.06 & $9.10 \mathrm{E}-04$ & $4910 \pm 294$ & $-0.16 \pm 0.06$ \\
\hline & 0.07 & $1.06 \mathrm{E}-03$ & $4210 \pm 129$ & $-0.22 \pm 0.03$ \\
\hline \multirow[t]{6}{*}{$4.1 \times 10^{-2}$} & 0.02 & $3.05 \mathrm{E}-04$ & $7115 \pm 148$ & 0 \\
\hline & 0.03 & $4.54 \mathrm{E}-04$ & $6510 \pm 445$ & $-0.03 \pm 0.05$ \\
\hline & 0.04 & $6.03 \mathrm{E}-04$ & $5398 \pm 340$ & $-0.12 \pm 0.05$ \\
\hline & 0.05 & $7.56 \mathrm{E}-04$ & $4531 \pm 372$ & $-0.13 \pm 0.07$ \\
\hline & 0.06 & $9.07 \mathrm{E}-04$ & $3888 \pm 165$ & $-0.10 \pm 0.04$ \\
\hline & 0.07 & $1.06 \mathrm{E}-03$ & $3694 \pm 238$ & $-0.27 \pm 0.06$ \\
\hline
\end{tabular}


Table 10: $\mathrm{K}$ and $\mathrm{N}_{\text {blob }}$ values obtained at various salt concentrations

\begin{tabular}{|c|c|c|c|}
\hline & Salt Concentration $(\mathrm{M})$ & $N_{\text {blob }}$ (in base pairs) & $\mathrm{K}\left(\mathrm{M}^{-1}\right)$ \\
\hline \multirow{4}{*}{$\begin{array}{c}\text { DNA-EB quenched by Cu } \\
\text { cations with } \mathrm{Na}_{2} \mathrm{SO}_{4} \text { solution }\end{array}$} & $5.0 \mathrm{E}-04$ & $8.9 \pm 0.9$ & $32952 \pm 93500$ \\
\cline { 2 - 4 } & $5.0 \mathrm{E}-03$ & $10.5 \pm 1.3$ & $3549 \pm 1780$ \\
\cline { 2 - 4 } & $7.5 \mathrm{E}-03$ & $6.8 \pm 0.6$ & $6123 \pm 3280$ \\
\cline { 2 - 4 } & $1.25 \mathrm{E}-02$ & $5.1 \pm 0.3$ & $9765 \pm 5300$ \\
\cline { 2 - 4 } & $2.1 \mathrm{E}-02$ & $4.8 \pm 0.4$ & $3005 \pm 900$ \\
\cline { 2 - 4 } & $3.0 \mathrm{E}-02$ & $3.1 \pm 0.2$ & $3933 \pm 1007$ \\
\cline { 2 - 4 } & $4.0 \mathrm{E}-02$ & $2.9 \pm 0.2$ & $2054 \pm 380$ \\
\cline { 2 - 4 } & $5.0 \mathrm{E}-04$ & $9.9 \pm 0.8$ & $8210 \pm 5487$ \\
\cline { 2 - 4 } $\begin{array}{c}\text { DNA-EB quenched by Ni }{ }^{2+} \\
\text { cations with } \mathrm{Na}_{2} \mathrm{SO}_{4} \text { solution }\end{array}$ & $5.0 \mathrm{E}-03$ & $9.3 \pm 0.8$ & $2695 \pm 733$ \\
\cline { 2 - 4 } & $1.25 \mathrm{E}-02$ & $5.2 \pm 0.5$ & $2598 \pm 802$ \\
\cline { 2 - 4 } & $2.1 \mathrm{E}-02$ & $3.6 \pm 0.3$ & $2046 \pm 529$ \\
\hline \multirow{3}{*}{$\begin{array}{c}\text { DNA-EB quenched by Cu } \\
\text { cations with } \mathrm{NaCl}^{2+}\end{array}$} & $3.0 \mathrm{E}-02$ & $2.4 \pm 0.1$ & $4769 \pm 1388$ \\
\cline { 2 - 4 } & $9.9 \mathrm{E}-03$ & $9.1 \pm 1.0$ & $2753 \pm 1050$ \\
\cline { 2 - 4 } & $2.5 \mathrm{E}-02$ & $6.2 \pm 0.5$ & $2703 \pm 672$ \\
\hline
\end{tabular}

